\title{
A numerical approach for simulating fluid structure interaction of flexible thin shells undergoing arbitrarily large deformations in complex domains
}

\author{
Anvar Gilmanov $^{a}$, Trung Bao Le ${ }^{a}$, Fotis Sotiropoulos ${ }^{a, b}$ \\ ${ }^{a}$ Saint Anthony Falls Laboratory \\ University of Minnesota \\ Minneapolis, MN 55414 \\ ${ }^{\mathrm{b}}$ Department of Civil Engineering \\ University of Minnesota \\ Minneapolis, MN 55414
}

\begin{abstract}
We present a new numerical methodology for simulating fluid-structure interaction (FSI) problems involving thin flexible bodies in an incompressible fluid. The FSI algorithm uses the Dirichlet-Neumann partitioning technique. The curvilinear immersed boundary method (CURVIB) is coupled with a rotation-free finite element (FE) model for thin shells enabling the efficient simulation of FSI problems with arbitrarily large deformation. Turbulent flow problems are handled using largeeddy simulation with the dynamic Smagorinsky model in conjunction with a wall model to reconstruct boundary conditions near immersed boundaries. The CURVIB and FE solvers are coupled together on the flexible solid-fluid interfaces where the structural nodal positions, displacements, velocities and loads are calculated and exchanged between the two solvers. Loose and strong coupling FSI schemes are employed enhanced by the Aitken acceleration technique to ensure robust coupling and fast convergence especially for low mass ratio problems. The coupled CURVIBFE-FSI method is validated by applying it to simulate two FSI problems involving thin flexible structures: 1) vortex-induced vibrations of a cantilever mounted in the wake of a square cylinder at different mass ratios and at low Reynolds number; and 2) the more challenging high Reynolds number problem involving the oscillation of an inverted elastic flag. For both cases the computed results are in excellent agreement with previous numerical simulations and/or experiential measurements. Grid convergence tests/studies are carried out for both the cantilever and inverted flag problems, which show that the CURVIB-FE-FSI method provides their convergence. Finally, the capability of the new methodology in simulations of complex cardiovascular flows is demonstrated by applying it to simulate the FSI of a trileaflet, prosthetic heart valve in an anatomic aorta and under physiologic pulsatile conditions.
\end{abstract}


Key words: fluid-structure interaction, immersed boundary method, finite element, thin shells, rotation-free approach

\section{Introduction}

Fluid-structure interaction problems involving three-dimensional, thin, flexible structures are encountered in a broad range of problems in engineering and biology. Examples include, among others, inflating parachutes, energy harvesting devices, swimming aquatic organisms, and native and prosthetic heart valves. Such problems occur across a broad range of Reynolds numbers and flow regimes, often take place in geometrically complex domains, and commonly involve arbitrarily large deformations of the flexible structures. These attributes, and especially the highly non-linear nature of the ensuing FSI, present unique challenges to numerical methods for simulating such problems. Such challenges arise from, among others, i) the need to model geometric and hyperelastic non-linearities of the solid bodies; ii) the complexity of the flow domains and the arbitrarily large amplitude of the deformation thin flexible structures may undergo; and iii) the challenges in obtaining robust and efficient FSI algorithms especially in problems with low mass ratios $[1,2]$, which are commonly encountered in heart valve simulations. These challenges along with approaches that have previously been developed in the literature for tackling them are discussed in more detail below.

There are two general approaches for simulating complex flows with deformable boundaries: the boundary conforming Arbitrary Lagrangian Eulerian (ALE) approach and Immersed Boundary (IB) methods. The ALE approach [3,4] is well suited for simulating high Reynolds number flows due to its inherent body-fitted mesh structure that conforms to boundaries at all times. However, ALE methods are cumbersome to apply to problems with large deformations since they require frequent remeshing in order to prevent the mesh from becoming severely distorted when large deformation develop. The remeshing procedure is computationally expensive making ALE methods difficult to apply in complex three-dimensional problems. Fixed, non-boundary conforming, grid methods provide another alternative to solving problems with deformable boundaries and complex geometry. Such methods are generally referred to as immersed boundary (IB) methods and are especially attractive for simulations of complex flows in engineering and biology because they do not require remeshing and can readily handle arbitrarily large deformations. The various types of IB methods have been recently reviewed by Sotiropoulos and Yang [1]. The interested reader is referred to this paper as well as the earlier review by Mittal and Iaccarino [5] for details. Here we focus our literature review exclusively on numerical approaches proposed for handling FSI of flexible structures in complex domains. In our

Email addresses: agilmano@umn.edu (Anvar Gilmanov), lebao002@umn.edu (Trung Bao Le), fotis@umn.edu (Fotis Sotiropoulos). 
review, we distinguish and pay special attention to the discretization techniques used to handle the flow and structural governing equations since a range of formulations have been proposed. These include pure finite-difference (FD) $[6,7,8,9,10]$ or finite-element (FE) $[11,12,13]$ methods for both the flow and structural equations as well as mixed formulations combining FD (or finite volume) discretization for the flow with FE for the structural equations $[14,15]$.

Diffused-interface IB methods use finite-differencing for both the fluid and structural solvers [6] due to the simplicity in obtaining the loading condition on the structural surface by incorporating appropriately defined body forces in the governing equations. A number of successful applications of such IB-FD-FD methods have been reported over the years $[8,9,7]$. The accuracy of such methods can be improved by incorporating local mesh refinement as was done in [6]. One potential difficulty with this class of methods, however, arises from treating the solid surface as diffused interface, which complicates the calculation of the details of the wall shear stress field on the surface. Yet, such detailed calculations may be required in heart valve flow simulations, where complex wall shear stress patterns on the valve leaflets have been linked with increased potential for aortic valve disease [16].

A sharp-interface IB method using FD formulations for both the flow and structural equations was proposed by Luo et al. [10]. This method was formulated for solving linear viscoelastic solids and applied to simulate two-dimensional FSI in laryngeal aerodynamics. The same formulation was later modified to incorporate a FE formulation for the structural equations and applied to simulate FSI of a high mass ratio 3D flapping wing at very low Reynolds number $(R e=50)$ [17]. Fung-Bao et al. [18] further extended this method to simulate several complex FSI problems at low Reynolds numbers $\left(R e \sim 10^{2}\right)$. An ALE formulation utilizing a so-called embedded boundary approach was proposed by Farhat and K.Lakshminarayan [15] for solving compressible FSI problems for external aerodynamics applications at high Reynolds numbers. This approach employs finite volume discretization for the fluid equations with finite elements for the structural equations. While this approach can work well for structures in unbounded domains, remeshing difficulties may arise when the structure is embedded within a complex confined domain. A pure finite-element based formulation, for both the flow and the structural equations, was recently proposed by Kamensky et al. [19]. This method employs the an immersogeometric FSI approach and was applied to simulate FSI of a bioprosthetic heart valve in a straight aorta.

In FSI simulations of biological tissues, e.g. heart valve leaflet interaction with blood flow, it is critical to use a relevant and efficient structural model that is able to realistically represent the deformation of the tissue under loads imposed by the pulsatile blood flow. Such undertaking, however, is not a trivial task since the large deformations of the tissue and its underlying geometric non-linearity pose major modelling challenges. To circumvent these challenges recent studies attempting to simulate FSI of tissue valves chose to either use simplified membrane-like materials [20] or treat the valve leaflets as thick bodies [18]. However, biological tissues of leaflets are normally thin and they exhibit significant 
bending. Therefore, a shell model for the solid body is a more appropriate choice [21, 19]. Most finite-element (FE) methodologies for handling shells, however, are computationally very demanding as they employ two or three nodal rotations alongside with three nodal translations, i.e. 5 or 6 degree of freedom per node. An exhaustive review of this large body of literature is beyond the scope of this paper but the reader is referred to a number of recent review papers on the topic [22, 23]. Note that the efficiency of the FE shell model becomes of paramount concern in FSI simulations of complex problems where the need to couple the fluid and structural solvers together can dramatically increase the computational cost per time step. For that, in this work we adapt and incorporate in the FSI methodology a previously developed nonlinear, rotation-free triangular shell element formulation [24], which has already been shown to provide accurate and robust solutions of various thin shell FE problems. Such an approach, however, has not been coupled before with a flow solver to simulate FSI problems and it is this coupling that constitutes one of the important contributions of our work.

The main contribution of this work is to develop a new numerical approach for the efficient simulation of complex FSI problems involving thin flexible structures, such as those occurring in a range of cardiovascular, biological and energy harvesting applications. We propose to integrate our previously developed curvilinear immersed boundary (CURVIB) FSI method for rigid bodies [25, 26, 27, 28] with an efficient rotation-free FE shell model [24]. The CURVIB method employs second-order accurate, central finite differencing discretization for the flow equations along with an efficient fractional-step approach for satisfying the discrete continuity equation to machine zero in curvilinear grids. The method has also been extended to carry out large-eddy simulation (LES) of turbulent flows using wall models for reconstructing boundary conditions at the immersed boundary nodes [29]. The LES version of the CURVIB method has been validated extensively for a broad rage of complex turbulent flows. Some recent examples include: turbulent flow past an axial flow turbine in an open channel [30]; open-channel turbulence interacting with a mobile sediment bed [31], and complex rigid structures interacting with a free surface [28]. As such the CURVIB method provides an efficient and accurate approach for simulating geometrically complex flows across a range of Reynolds numbers. Furthermore, and since the CURVIB method employs unstructured triangular meshes to discretize immersed boundaries, the method is readily suited for coupling it with a FE solver for the structural equations. To enable this coupling, we report herein a number of algorithmic advances and significant improvements of our previously developed methodology. More specifically, we report a new methodology for representing and tracking the motion of the thin structure as well as for efficiently calculating the loads on the fluid-structural interfaces in parallel environment. Since our FE solver is highly efficient for thin bodies, it can be applied for a variety of structures including engineering structures such as cantilevers, plates, beams or biological tissues such as heart valves and arterial walls. Another important contribution of this work is to demonstrate the ability of the resulting methodology to simulate a very challenging FSI problem involving the large amplitude oscillations of an inverted elastic flag. This problem has been recently studied experimentally by Kim et al. [32] and to our knowledge it has not been simulated before numerically. It is especially challenging because: 1) the flow occurs at high Reynolds number and for that requires 
implementing the resulting CURVIB-FE-FSI formulation in conjunction with large-eddy simulation (LES); and 2) depending on the elasticity of the flag the FSI problem exhibits dynamically rich solutions [32]. Here we report simulations for a set of parameters for which the flag undergoes periodic oscillations and show that the computed motion of the flag is in excellent agreement with the measurements. We also demonstrate for the first time the ability of the coupled CURVIB-FE-FSI method to simulate the FSI of a tri-leaflet valve in an anatomic aorta. Our simulations capture the rich $3 \mathrm{D}$ vorticity dynamics during the opening and closing of the valve leaflets.

The paper is organized as follows. In Section 2, we describe the governing equations for both fluid and solid structure. In Section 3 we present the numerical approach used to solve the coupled system of fluid and solid equations with appropriate boundary conditions. Two validation problems for FSI algorithm are presented in Section 4. The first such problem is that of cantilever oscillation under the action of vortex shedding from a square cylinder. Comparisons between the computed results with previous computational data show very good agreement. The second problem is the flapping of an inverted flag [32], which is simulated using LES with the Smagorinsky dynamic model and the wall model approach developed for the CURVIB method by Kang et al. [33]. The computed results are shown to be in excellent agreement with the experiential data of Kim et al. [32]. In this section we also demonstrate the applicability of the proposed FSI approach to simulate pulsatile blood flow in an anatomic aorta with a tri-leaflet heart valve at physiologic conditions. Finally, in the Section 5 we summarize the major findings of this work and future extension are discussed.

\section{Governing equations}

We consider fluid-structure interaction of a deformable body $\Omega_{s}$ submerged in an incompressible fluid occupying a volume $\Omega_{f}$ bounded by $\partial \Omega_{f}$ as shown in Figure 1 - the method is applicable to multiple deformable thin bodies but for the ease of presentation and without loss of generality we present the method for a single body.

In what follows, we use bold symbols for vectors and bold symbols with two underlines for tensors and matrices. The regular and italic symbols are reserved for scalar and vector/tensor components, respectively. The over bar notation indicates known and/or prescribed values.

\subsection{The equations for the fluid domain}

In general fluid boundaries can be presented as consisting of three non-overlapping parts: $\partial \Omega_{f}=\Gamma_{f}^{N} \cup \Gamma_{f}^{D} \cup \Gamma^{f s i}$. Here $\Gamma_{f}^{D}$ and $\Gamma_{f}^{N}$ are the stationary boundaries in which Dirichlet and/or Neumann boundary conditions are specified. As above-mentioned, $\Gamma^{f s i}$ is the 
interface between the fluid domain and the solid domain, i.e the moving interface the configuration of which needs to be determined by solving the FSI problem.

The equations governing the motion of Newtonian incompressible fluid in a domain $\Omega_{f}$ as the Navier-Stokes and continuity equations, which read in vector/tensor notation as follows:

$$
\begin{aligned}
& \rho_{f} \frac{d \mathbf{v}}{d t}=\nabla \cdot \underline{\underline{\boldsymbol{\sigma}_{f}}} \text { in } \Omega_{f}, \\
& \nabla \cdot \mathbf{v}=0 \text { in } \Omega_{f} .
\end{aligned}
$$

In the above equations, $\rho_{f}$ is the mass density of the fluid, $d / d t$ is the material or Lagrangian time derivative, $\mathbf{v}$ is the fluid velocity vector and $\boldsymbol{\sigma}_{f}$ is the stress tensor. The above equations are subjected to various boundary conditions for the velocity $\mathbf{v}$ on the various segments comprising the fluid boundary. For example, on the Dirichlet portion of the boundary $\Gamma_{f}^{D}$ Dirichlet boundary conditions:

$$
\mathbf{v}=\overline{\mathbf{v}} \quad \text { on } \quad \Gamma_{f}^{D}
$$

On the Neumann segment of the boundary, a stress boundary condition of the following form may be applied:

$$
\underline{\underline{\boldsymbol{\sigma}_{f}}} \cdot \mathbf{n}_{f}=\overline{\mathbf{t}}_{f} \quad \text { on } \quad \Gamma_{f}^{N}
$$

where $\overline{\mathbf{v}}$ and $\overline{\mathbf{t}}_{f}$ are known functions, $\mathbf{n}_{f}$ is the normal unit vector to the $\Gamma_{f}^{N}$ boundary.

For FSI problems, the immersed deformable body has its own displacement field $\mathbf{u}$, velocity field $\dot{\mathbf{u}}$ and stress field $\underline{\underline{\boldsymbol{\sigma}_{s}}}$. On the $\Gamma^{f s i}$ boundary between the solid and fluid domains, the velocity field and the $\overline{\bar{n}}$ rmal stress field must be continuous. This physical requirement gives rise to the following set of boundary conditions on the FSI segment of the fluid boundary:

$$
\begin{aligned}
& \mathbf{v}=\dot{\mathbf{u}} \text { on } \Gamma^{f s i} \\
& \underline{\underline{\boldsymbol{\sigma}_{f}}} \cdot \mathbf{n}_{f}=\underline{\underline{\boldsymbol{\sigma}_{s}}} \cdot \mathbf{n}_{s} \text { on } \Gamma^{f s i}
\end{aligned}
$$

Here $\boldsymbol{n}_{f}$ and $\boldsymbol{n}_{s}$ are the local normal unit vectors on the fluid and solid interface, respectively. Note, therefore, that on the $\Gamma^{f s i}$ segment of the boundary both Dirichlet and Neumann conditions must be satisfied (given by Eq. (4)), so that the problem is well posed and the Navier-Stokes equations (1) supplied with boundary conditions (2) and (3) on $\Gamma_{f}^{D}$ and $\Gamma_{f}^{N}$ can be solved.

To facilitate the subsequent presentation of the FSI algorithm, we denote the governing equations for the fluid domain as an operator $\mathfrak{F}$, which receives the input information from the boundary conditions and yields the pressure $p$ and velocity field $\mathbf{v}$ inside the fluid domain $\Omega_{f}$ as follows:

$$
(p, \mathbf{v})=\mathfrak{F}\left(\overline{\mathbf{v}}, \overline{\mathbf{t}}_{f}, \dot{\mathbf{u}}, \underline{\underline{\boldsymbol{\sigma}_{s}}}\right) \text { in } \Omega_{f}
$$

here $\dot{\mathbf{u}}$ and $\underline{\underline{\boldsymbol{\sigma}_{s}}}$ are applied at the boundary $\Gamma^{f s i}$. Equation 5, therefore, should be viewed 
as the operator notation for equations $1,2,3$ and 4 .

\subsection{The equations for the solid domain}

In the solid domain we use the Lagrangian viewpoint to describe the motion of a material point. The current position $\mathbf{r}$ of a material point at time $t$ is related to its position at the reference configuration $\mathbf{R}$ by the mapping $\boldsymbol{\Phi}$ :

$$
\mathbf{r}=\boldsymbol{\Phi}(\mathbf{R})
$$

The gradient tensor of the transformation is therefore :

$$
\underline{\underline{\mathbf{F}}}=\frac{\partial \Phi}{\partial \mathbf{R}}
$$

The displacement of a material point is defined as:

$$
\mathbf{u}=\mathbf{r}-\mathbf{R}
$$

and the velocity of the material point is given as:

$$
\dot{\mathbf{u}}=d \mathbf{u} / d t
$$

The momentum equations for the solid part, formulated in the current configuration have the following form:

$$
\rho_{s} \frac{d \dot{\mathbf{u}}}{d t}=\nabla \cdot \underline{\underline{\boldsymbol{\sigma}_{s}}}+\rho_{s} \mathbf{b}
$$

where $\rho_{s}$ is the current mass density of the material.

Here $\boldsymbol{\sigma}_{s}$ is the Cauchy stress tensor, which represents the gradient operator in the current configuration, and $\mathbf{b}$ is the body force per unit mass. The boundary of the the solid structure can be represented as sum of non-overlapping parts $\partial \Omega_{s}=\Gamma_{s}^{N} \cup \Gamma_{s}^{D} \cup \Gamma^{f s i}$, where the indices $D$ and $N$ denote boundaries with Dirichlet and Neumann conditions respectively:

$$
\begin{aligned}
& \dot{\mathbf{u}}=\overline{\mathbf{u}} \quad \text { on } \quad \Gamma_{s}^{D}, \\
& \underline{\underline{\boldsymbol{\sigma}_{s}}} \cdot \mathbf{n}_{s}=\overline{\mathbf{t}}_{s} \text { on } \Gamma_{s}^{N},
\end{aligned}
$$

boundaries $\Gamma_{s}^{D}$ and $\Gamma_{s}^{N}$ represent the portions of the surface of the body in its current configuration where Dirichlet and Neumann conditions are applied, respectively, $\overline{\mathbf{t}}_{s}$ is a traction vector acting on the surface, $\mathbf{n}_{s}$ is a unit normal to the boundary and $\overline{\mathbf{u}}$ is the velocity prescribed on the surface. 
For FSI problems additional boundary conditions must be implemented on the $\Gamma^{f s i}$ :

$$
\begin{aligned}
& \dot{\mathbf{u}}=\mathbf{v} \quad \text { on } \quad \Gamma^{f s i}, \\
& \underline{\underline{\boldsymbol{\sigma}_{s}}} \cdot \mathbf{n}_{s}=\mathbf{t}_{f}, \quad \text { on } \quad \Gamma^{f s i},
\end{aligned}
$$

here $\Gamma^{f s i}$ is part of the moving structure surface the configuration of which needs to be determined by solving the FSI problem, $\mathbf{t}_{f}=\boldsymbol{\sigma}_{f} \cdot \mathbf{n}_{f}$ is a traction vector which acts on this part of surface from the fluid, $\boldsymbol{\sigma}_{f}$ and $\mathbf{n}_{f} \overline{\overline{\text { are }}}$ the stress tensor and surface normal unit vector from the fluid. We will discuss later how to define the traction vector for thin surfaces.

The solid momentum equations with the boundary conditions can be recasted in terms of an operator $\mathfrak{S}$, which incorporates both the (kinematic and dynamic) boundary conditions and constitutive equations to yield the velocity $\dot{\mathbf{u}}$ and displacement field $\mathbf{u}$

$$
(\mathbf{u}, \dot{\mathbf{u}})=\mathfrak{S}\left(\overline{\mathbf{v}}, \overline{\mathbf{t}}, \mathbf{v}, \mathbf{t}_{f}\right) \text { in } \Omega_{s},
$$

here $\mathbf{v}$ and $\mathbf{t}_{f}$ are applied at the boundary $\Gamma^{f s i}$.

\section{Numerical algorithms for Fluid-Structure Interaction}

An IB algorithm for solving FSI problems with thin deformable structures embedded in a fluid domain requires developing and integrating the following algorithmic components: (1) an algorithm for solving the fluid flow equations (Sec. 3.1); (2) an algorithm for solving the thin shell structural equations (Sec. 3.2); (3) an approach for defining the action from the thin shell onto the surrounding fluid by identifying the IB nodes in the vicinity of the body where boundary conditions need to reconstructed (Sec. 3.3); (4) an approach for calculating the action from the fluid to the thin shell body computing the forces due to pressure and shear (Sec. 3.4); and (5) an algorithm that integrates the fluid and solid solvers into a coupled FSI formulation. In this section we discuss the approaches we adopt in this work to develop these algorithmic components (Sec. 3.5).

\subsection{The fluid solver $\mathfrak{F}$}

The fluid solver is based on the CURVIB approach [26] which uses the hybrid stagger/nonstaggered approach originally proposed by Gilmanov and Sotiropoulos [25] to solve the governing equations in generalized curvilinear grids [26]. The Navier-Stokes and continuity equations (1) are partially transformed in generalized curvilinear coordinates and read in 
tensor form (repeated indices $j=1,3$ assumes summation) as follows:

$$
\begin{aligned}
& \frac{\partial}{\partial \xi^{j}}\left(\frac{V^{j}}{J}\right)=0 \\
& \frac{\partial v_{q}}{\partial t}+C\left(v_{q}\right)+G_{q}(p)-\frac{1}{R e} D\left(v_{q}\right)=0, \quad q=1,2,3
\end{aligned}
$$

where the Cartesian velocity vector is denoted as $\mathbf{v}\left(v_{1}, v_{2}, v_{3}\right), p$ is the pressure divided by the density $\rho_{f}, V^{j}=v_{r} \xi_{x_{r}}^{j}$ is the $j^{\text {th }}$ contravariant velocity component in the general curvilinear coordinate system $\xi\left(\xi^{1}, \xi^{2}, \xi^{3}\right), J$ is the Jacobian of the geometric transformation $J=\partial\left(\xi^{1}, \xi^{2}, \xi^{3}\right) / \partial\left(x_{1}, x_{2}, x_{3}\right)$, and $g^{r m}=\xi_{x_{q}}^{r} \xi_{x_{q}}^{m}$ is the contravariant metric tensor. The convective $C\left(v_{q}\right)$, gradient $G_{q}(p)$ and viscous $D\left(v_{q}\right)$ operators in Eqn (14) are defined in curvilinear coordinates as (the repeated indexes $r, m$ imply summation over the values $1,2,3)$ :

$$
\begin{aligned}
C\left(v_{q}\right) & =J \frac{\partial}{\partial \xi^{r}}\left(\frac{V^{r}}{J} v_{q}\right), \quad q=1,2,3 \\
D\left(v_{q}\right) & =J \frac{\partial}{\partial \xi^{r}}\left(\frac{g^{r m}}{J} \frac{\partial v_{q}}{\partial \xi^{m}}\right) \\
G_{q}(p) & =J \frac{\partial}{\partial \xi^{r}}\left(\frac{\xi_{x_{q}}^{r}}{J} p\right)
\end{aligned}
$$

The above equations are discretized via a hybrid staggered/non-staggered approach using three-point central differencing for all spatial derivatives and integrated in time via a second-order accurate fractional step, pressure projection method. The momentum equations are solved with a Jacobian-free solver while Flexible Generalized Minimal Residual (FGMRES) method with multigrid pre-conditioner is used to solve the Poisson equation to satisfy the discrete continuity equation to machine zero (see Ge and Sotiropoulos [26] for details).

Complex immersed boundaries are handled using a sharp-interface IB method with velocity reconstruction along the local normal to the body [25, 26, 27]. The details about the reconstruction method for thin flexible boundaries will be provided in a subsequent section of this paper. For more details about the various aspects of the CURVIB method, the discretization method, and the iterative solvers we employ to solve the governing equations the interested reader is referred to [26, 27], and [1].

The CURVIB method has been recently extended to carry out large-eddy simulation (LES) of geometrically complex turbulent flows. The details of the LES version of our flow solver can be found in [29] and [30]. Here it suffices to mention that the dynamic Smagorinsky model [34] is used for subgrid-scale closure with three-point central, second-order accurate finite differencing for the convective terms. Boundary conditions at IB nodes in the vicinity of complex immersed boundaries are reconstructed using a wall model approach adapted for the CURVIB method by Kang et al. [29]. The resulting LES-CURVIB solver has been validated extensively for a number of complex turbulent flows dominated by energetic coherent vortices. The validation study most relevant to the simulations we will report in 
this paper is the work of [30] who simulated turbulent flow past a complete hydrokinetic turbine in an open channel. The simulation resolves all geometrical details of the turbine, including the pylon, the nacelle and the rotating turbine blades. Comparisons of mean flow and turbulence statistics in the wake of the turbine with experimental data showed very good agreement. In this paper we will report the first application of the LES version of method to simulate FSI of a flexible structure at high Reynolds number.

\subsection{The solid solver $\mathfrak{S}$ : finite element model for thin shells}

The momentum equations for the solid (Eqs. 10) can be expressed in various weak formulations using the principle of virtual work. In this work we select the Lagrangian weak form, which is related to the initial configuration and uses the second Piola-Kirchhoff stress tensor $\underline{\underline{\mathbf{S}}}$ and the variation of the Green-Lagrange strain tensor $\underline{\underline{\mathbf{E}}}$. By virtue of how they appear in the principle of virtual work given below, they constitute a dual set written in the reference configuration for volume $V_{0}$ with surface boundary $A_{0}$ as follows:

$$
\int_{V_{0}}\left(\delta \underline{\underline{\mathbf{E}}}^{T} \underline{\underline{\mathbf{S}}}+\delta \mathbf{u}^{T} \rho_{s} \ddot{\mathbf{u}}\right) d V_{0}-\int_{A_{0}} \delta \mathbf{u}^{T} \mathbf{t}_{0} d A_{0}=0 .
$$

In the above equation, $\rho_{s}$ is the structure density in the original configuration (for incompressible structure material the density in both the initial and current configurations is the same), $\mathbf{t}_{0}$ are the surface loads in that configuration, and $\ddot{\mathbf{u}}$ is the acceleration. The Neo-Hookean [35] constitutive equation in a local Cartesian system is used to relate the stress and strain tensors as follows:

$$
\underline{\underline{\mathbf{S}}}^{l o c}=\underline{\underline{\mathbf{D}}}^{l o c} \underline{\underline{\mathbf{E}}}^{l o c}
$$

with

$$
\underline{\underline{D}}^{l o c}=\frac{E}{1-\nu^{2}}\left[\begin{array}{ccc}
1 & \nu & 0 \\
\nu & 1 & 0 \\
0 & 0 & \frac{1-\nu}{2}
\end{array}\right],
$$

where $E$ is the Young's modulus and $\nu$ is the Poisson's ratio, index $l o c$ indicates that constitutive equation is described in a local Cartesian system. The well known transformation matrix $\underline{\underline{\mathrm{Q}}}[24]$ is used to define stress and strain tensors in the local Cartesian system through tensors in natural/curvilinear system, for example, $\underline{\underline{\mathbf{E}}}^{l o c}=\underline{\underline{\mathrm{QE}}} \underline{\underline{ }}$.

We consider only thin shell models for the solid domain. In the Kirchhoff-Love model of thin shells [36] the position vector $\mathbf{R}$ of any point within the shell in the reference configuration is defined in terms of the surface curvilinear coordinates and the local normal distance $\zeta$ to the middle surface, with $-h_{0} / 2 \leq \zeta \leq h_{0} / 2$, where $h_{0}$ is a thickness of the shell. The position of the points in the current configuration of the shell $\mathbf{r}$ can be mapped back to the reference configuration using the same local normal distance to the 
middle surface $\zeta$. For the Kirchhoff-Love model of thin shells the components of the Green-Lagrange strain tensor in the entire volume of the shell can be expressed via the deformation of the shell's middle surface as follows:

$$
E_{i j}=E_{i j}^{m}+\zeta E_{i j}^{b}
$$

Here $E_{i j}^{m}$ are the membrane and $E_{i j}^{b}$ the bending components of the strain tensor.

We adopt herein the model developed by Stolarski et al. [24], which approximates the shell curvature tensor, associated with a main element and three surrounding elements, using a complete quadratic polynomial in a moving with the shell rectilinear coordinate system attached to that element. This approach permits for simple, albeit accurate, approximations in the evaluation of the element curvature tensor. Most importantly, since the above approach is used only to compute the bending strains within the element the computation of the membrane strains is based on the flat geometry of the element - non-physical membrane locking is automatically avoided. For the derivation and the details of the method the reader is referred to Stolarski et al. [24].

Let us now introduce the discretized version of the governing equations for the structure by the $\mathrm{FE}$ method in a triangulated domain consisting of $e=1, \bar{E}$ elements (where $e$ denotes a triangular element and $\bar{E}$ is the number of elements discretizing the domain). By virtue of Eqs. (19), the weak formulation of Eqs. (10) can be written in the following form, by summing over all elements $e$ of the triangulated domain:

$$
\begin{gathered}
\sum_{e=1}^{\bar{E}}\left(\int_{V_{0 e}}\left(\delta \mathbf{u}_{e}^{T}\left(\underline{\underline{\mathbf{B}}}^{m}+\zeta \underline{\underline{\mathbf{B}}}^{b}\right)^{T} \underline{\underline{\mathbf{S}}}-\delta \mathbf{u}_{e}^{T} \rho_{s} \mathbf{b}+\delta \mathbf{u}_{e}^{T} \rho_{s} \ddot{\mathbf{u}}_{e}\right) d V_{0}-\right. \\
\left.-\int_{A_{0 e}} \delta \mathbf{u}_{e}^{T} \mathbf{t} d A_{0}\right)=0
\end{gathered}
$$

where $\mathbf{u}_{e}, \ddot{\mathbf{u}}_{e}$ are the displacement and acceleration of the triangular element $e, \underline{\underline{\mathbf{B}}}^{m}$ and $\underline{\underline{\mathbf{B}}}^{b}$ are membrane and bending strain-displacement matrices, respectively. Thus, the vector of internal forces is

$$
\mathbf{f}_{e}^{i n t}=\int_{V_{0 e}}\left[\left(\underline{\underline{\mathbf{B}}}^{m}\right)^{T} \underline{\underline{\mathbf{Q}}}^{T} \underline{\underline{\mathbf{D}}}^{l o c} \underline{\underline{\mathbf{Q}}}^{m}+\zeta^{2}\left(\underline{\underline{\mathbf{B}}}^{b}\right)^{T} \underline{\underline{\mathbf{Q}}}^{T} \underline{\underline{\mathbf{D}}}^{l o c} \underline{\underline{\mathbf{Q}}}^{b}\right] d V_{0},
$$

where superscript indices $(m)$ and $(b)$ indicate membrane and bending matrices in the curvilinear coordinate system on the surface [24]. Because of space restrictions, we do not give herein the detailed formulation of all matrices we employ. Instead we refer the readers to the recently published paper [24], where we give all formulations and matrices we employ in the nonlinear thin shell approach.

The element vector of the nodal external forces $\mathbf{f}_{e}^{e x t}$ and the element mass matrix $\underline{\underline{\mathbf{M}_{e}}}$ take the following form

$$
\mathbf{f}_{e}^{e x t}=\int_{A_{0 e}} \mathbf{N}^{T} \mathbf{t} d A_{0}
$$




$$
\underline{\underline{\mathbf{M}_{e}}}=\int_{V_{0 e}} \rho_{s} \mathbf{N}^{T} \mathbf{N} d V_{0}
$$

where $\boldsymbol{t}$ is a traction vector, and $\mathbf{N}$ is a vector of linear basis functions [24]. Here the external forces $\mathbf{f}^{e x t}(\mathbf{u}, \mathbf{t})$ depend both on structure displacements $\mathbf{u}$ and the applied fluid traction t. Assembly of the above vectors and matrices leads to the following final form of the structural domain equations:

$$
\mathbf{f}^{i n t}(\mathbf{u})+\underline{\underline{\mathbf{M}}} \ddot{\mathbf{u}}=\mathbf{f}^{e x t}(\mathbf{u}, \mathbf{t})
$$

In this paper we used three types of boundary conditions for the shell: free, hinged and fixed boundary conditions. Detailed description and implementation of these boundary conditions one can find in [24].

In the numerical integration of some dynamic, nonlinear problems with high frequency modes a dissipative mechanism is needed in the Eq. (24) to dump spurious oscillations and help get converged solutions [37]. If dissipation is to be included in the system, a term related to the velocities has to be added in Eqn.(24) as follows:

$$
\mathbf{f}^{i n t}(\mathbf{u})+\underline{\underline{\mathbf{M}}} \ddot{\mathbf{u}}+\underline{\underline{D}} \dot{\mathbf{u}}=\mathbf{f}^{e x t}(\mathbf{u}, \mathbf{t}),
$$

where the matrix $\underline{\underline{\mathrm{D}}}$ presents the dissipation term.

The detailed description of the solid solver algorithm with all matrices, can be found in [24]. Here we give only a short description of this algorithm.

We employ the Newmark time integration algorithm [38] to solve solid-structure equation (25), which is formulated as follows:

$$
\begin{gathered}
\dot{\mathbf{u}}^{n+1}=\dot{\mathbf{u}}^{n}+\Delta t(1-\gamma) \ddot{\mathbf{u}}^{n}+\Delta t \gamma \ddot{\mathbf{u}}^{n+1}, \\
\mathbf{u}^{n+1}=\mathbf{u}^{n}+\Delta t \dot{\mathbf{u}}^{n}+\Delta t^{2}\left(\frac{1}{2}-\omega\right) \ddot{\mathbf{u}}^{n}+\Delta t^{2} \omega \ddot{\mathbf{u}}^{n+1},
\end{gathered}
$$

where $\Delta t$ is the time step, subscript $n, n+1$ indicates time level $t^{n+1}=t^{n}+\Delta t$, and $\gamma, \omega$ are parameters that determine the stability and accuracy of the scheme. Implicit schemes are unconditionally stable for

$$
2 \omega \geq \gamma \geq \frac{1}{2}
$$

The Newmark scheme has second order accuracy for $\gamma=0.5, \omega=0.25$ [37]. From Eq. (26) - (27) one gets the following formulas for the velocity and acceleration vectors

$$
\begin{gathered}
\ddot{\mathbf{u}}^{n+1}=\frac{1}{\omega \Delta t^{2}}\left(\mathbf{u}^{n+1}-\mathbf{u}^{n}\right)-\frac{1}{\omega \Delta t} \dot{\mathbf{u}}^{n}-\left(\frac{1}{2 \omega}-1\right) \ddot{\mathbf{u}}^{n}, \\
\dot{\mathbf{u}}^{n+1}=\frac{\gamma}{\omega \Delta t}\left(\mathbf{u}^{n+1}-\mathbf{u}^{n}\right)-\left(\frac{\gamma}{\omega}-1\right) \dot{\mathbf{u}}^{n}-\Delta t\left(\frac{\gamma}{2 \omega}-1\right) \ddot{\mathbf{u}}^{n},
\end{gathered}
$$


which, when inserted in Eq. (25), yield

$$
\begin{aligned}
& \mathbf{f}^{i n t}\left(\mathbf{u}^{n+1}\right)+\underline{\underline{\mathbf{M}}}\left[\frac{1}{\omega \Delta t^{2}}\left(\mathbf{u}^{n+1}-\mathbf{u}^{n}\right)-\frac{1}{\omega \Delta t} \dot{\mathbf{u}}^{n}-\left(\frac{1}{2 \omega}-1\right) \ddot{\mathbf{u}}^{n}\right]+ \\
& +\underline{\underline{\mathbf{D}}}\left[\frac{\gamma}{\omega \Delta t}\left(\mathbf{u}^{n+1}-\mathbf{u}^{n}\right)-\left(\frac{\gamma}{\omega}-1\right) \dot{\mathbf{u}}^{n}-\Delta t\left(\frac{\gamma}{2 \omega}-1\right) \ddot{\mathbf{u}}^{n}\right]=\mathbf{f}^{e x t} .
\end{aligned}
$$

When the unknown $\mathbf{u}^{n+1}$ is retained in the left hand side of the equation and the known variables are gathered in the right hand side, the following discrete equation is obtained

$$
\begin{aligned}
& \mathbf{f}^{\text {int }}\left(\mathbf{u}^{n+1}\right)+\frac{1}{\omega \Delta t^{2}} \underline{\underline{\mathbf{M}}} \mathbf{u}^{n+1}+\frac{\gamma}{\omega \Delta t} \underline{\mathbf{D}}^{n+1}= \\
& =\underline{\underline{\mathbf{M}}}\left[\frac{1}{\omega \Delta t^{2}} \mathbf{u}^{n}+\frac{1}{\omega \Delta t} \dot{\mathbf{u}}^{n}+\left(\frac{1}{2 \omega}-1\right) \ddot{\mathbf{u}}^{n}\right]+ \\
& +\underline{\underline{\mathbf{D}}}\left[\frac{\gamma}{\omega \Delta t} \mathbf{u}^{n}+\left(\frac{\gamma}{\omega}-1\right) \dot{\mathbf{u}}^{n}+\Delta t\left(\frac{\gamma}{2 \omega}-1\right) \ddot{\mathbf{u}}^{n}\right]+\mathbf{f}^{e x t} .
\end{aligned}
$$

The last equation constitutes a nonlinear system of algebraic equations that has to be solved at each time step. This system is solved using the Newton linearization approach. Denoting by $\mathbf{u}_{i}^{n+1}$ the value of $\mathbf{u}^{n+1}$ at iteration $i$, the following equation is obtained by linearizing $\mathbf{f}^{\text {int }}\left(\mathbf{u}_{i}^{n+1}\right)=\mathbf{f}^{i n t}\left(\mathbf{u}_{i-1}^{n+1}+\Delta \mathbf{u}_{i}^{n+1}\right)$ :

$$
\mathbf{f}^{i n t}\left(\mathbf{u}_{i}^{n+1}\right) \approx \mathbf{f}^{i n t}\left(\mathbf{u}_{i-1}^{n+1}\right)+\underline{\underline{\mathbf{K}_{i-1}^{n+1}}} \Delta \mathbf{u}_{i}^{n+1}=0,
$$

where $\underline{\mathbf{K}}$ is the tangent stiffness matrix. The increment $\Delta \mathbf{u}_{i}^{n+1}$ between the iteration $i-1$ and $i$ is the solution of the following system of linear algebraic equations, resulting from Eq. (32).

$$
\left(\underline{\underline{\mathbf{K}}}+\frac{1}{\omega \Delta t^{2}} \underline{\underline{\mathbf{M}}}+\frac{\gamma}{\omega \Delta t} \underline{\underline{\mathbf{D}}}\right)_{i-1}^{n+1} \Delta \mathbf{u}_{i}^{n+1}=\mathbf{R}_{i-1}^{n+1}
$$

with the residual

$$
\begin{aligned}
\mathbf{R}_{i-1}^{n+1} & =-\mathbf{f}^{i n t}\left(\mathbf{u}_{i-1}^{n+1}\right)-\left[\frac{1}{\omega \Delta t^{2}} \underline{\underline{\mathbf{M}}}+\frac{\gamma}{\omega \Delta t} \underline{\underline{\mathbf{D}}}\right] \mathbf{u}_{i-1}^{n+1}+ \\
& +\underline{\underline{\mathbf{M}_{i-1}^{n+1}}}\left[\frac{1}{\omega \Delta t^{2}} \mathbf{u}_{i-1}^{n}+\frac{1}{\omega \Delta t} \dot{\mathbf{u}}_{i-1}^{n}+\left(\frac{1}{2 \omega}-1\right) \ddot{\mathbf{u}}_{i-1}^{n}\right]+ \\
& +\underline{\underline{\underline{\mathbf{D}_{i-1}^{n+1}}}}\left[\frac{\gamma}{\omega \Delta t} \mathbf{u}_{i-1}^{n}+\left(\frac{\gamma}{\omega}-1\right) \dot{\mathbf{u}}_{i-1}^{n}+\Delta t\left(\frac{\gamma}{2 \omega}-1\right) \ddot{\mathbf{u}}_{i-1}^{n}\right]+\mathbf{f}^{e x t},
\end{aligned}
$$

in which the forces $\mathbf{f}^{e x t}(\mathbf{u}, \mathbf{t}), \mathbf{f}^{\text {int }}\left(\mathbf{u}^{n}\right)$ are computed according to the explicit formulas presented in the preceding sections. The matrix $\mathbf{D}$ presented in Eq. (25) can be independently defined as linear combination of mass and stiffness matrices $\underline{\underline{\mathbf{M}}}, \underline{\underline{\mathbf{K}}}$ (so-called proportional damping)

$$
\underline{\underline{\mathbf{D}}}=f_{m} \underline{\underline{\mathbf{M}}}+f_{k} \underline{\underline{\mathbf{K}}}
$$

where $f_{m}$ and $f_{k}$ are constants and are called "Rayleigh" damping coefficients [37].

The solution of the above linear equations, $\Delta \mathbf{u}_{i}^{n+1}$, is used to update displacements, 
velocities and accelerations as follows:

$$
\begin{aligned}
& \mathbf{u}_{i}^{n+1}=\mathbf{u}_{i-1}^{n+1}+\Delta \mathbf{u}_{i}^{n+1}, \\
& \dot{\mathbf{u}}_{i}^{n+1}=\frac{\gamma}{\omega \Delta t}\left(\mathbf{u}_{i}^{n+1}-\mathbf{u}_{i}^{n}\right)-\left(\frac{\gamma}{\omega}-1\right) \dot{\mathbf{u}}_{i}^{n}-\Delta t\left(\frac{\gamma}{2 \omega}-1\right) \ddot{\mathbf{u}}_{i}^{n}, \\
& \ddot{\mathbf{u}}_{i}^{n+1}=\frac{1}{\omega \Delta t^{2}}\left(\mathbf{u}_{i}^{n+1}-\mathbf{u}_{i}^{n}\right)-\frac{1}{\omega \Delta t} \dot{\mathbf{u}}_{i}^{n}-\left(\frac{1}{2 \omega}-1\right) \ddot{\mathbf{u}}_{i}^{n} .
\end{aligned}
$$

The iterative process is declared converged when a specified tolerance of the iterative process is met, and the algorithm is advanced to the next time level.

The Conjugate Gradient (CG) method [37] is used to solve the linear system of Eq. (34). Overall, the cost of using CG is relatively low. The overall computational cost, however, depends on the number of Newton iterations to update the displacement, velocity and acceleration of the nodal points of the structural mesh.

\subsection{Representation of immersed thin structures in the fluid domain}

As discussed above, a key feature of our method is that it employs a FE formulation for thin shells in which the structural equations are formulated and solved for the mid-surface of the thin structure (see section 3.2). This approach is selected herein because not only it is appropriate for handling thin structures but also augments the efficiency of the overall FSI methodology.

Whether the structure has thickness or not presents important algorithmic challenges for the CURVIB method. This is because the CURVIB method is designed to use normal vectors to the immersed surface to: $(i)$ identify the position of background grid nodes relative to the fluid/structure interface by finding fluid nodes (IB-nodes) in the immediate proximity of the interface as illustrated in Figure 3; (ii) reconstruct velocity boundary conditions at immersed boundary (IB) nodes along the local normal to the boundary; and (iii) calculate the loads on the structure imparted by fluid stresses for FSI problems (see section 3.4). Bodies with non-zero thickness, which have been handled in all previous applications of the CURVIB method, are closed surfaces (i.e. the topological equivalent for a sphere). This implies that at every point on the surface of the body there is a unique normal vector that points toward the fluid side of the interface, namely the positive wall normal vector (see Fig. 2a). On the other hand, when the structure is represented by its mid-plane, as in the present FE thin shell model, the resulting surface is what is referred to in topological terms as a surface with boundary (i.e. the topological equivalent of a disk). For such a case, it is readily apparent from Fig. $2 \mathrm{~b}$ that at each point on the surface both the positive and negative wall normal vectors point toward the fluid side of the interface. Consequently, the standard node classification and boundary condition reconstruction algorithms used in the CURVIB method [27] cannot be readily applied and need to be modified. In this section we describe the algorithmic changes we have implemented to the CURVIB method to enable its coupling with the thin shell FE formulation. 
At each triangular element $e$ on the mid-surface of the thin structure we calculate the positive $\mathbf{n}_{e}^{+}$and negative $\mathbf{n}_{e}^{-}$surface normal vectors to identify the positive and negative, respectively sides of the surface. The normal vectors are calculated at the center of the element $e$ and the positive normal is defined as the outward normal of the triangle with clockwise nodal numbering. It is thus evident that $\mathbf{n}_{e}^{+}=-\mathbf{n}_{e}^{-}$. The triangulated surface is tracked with a set of Lagrangian points (the nodes of the triangles), which are used to define the boundary conditions (position and velocity of each Lagrangian node) for the fluid solver.

To find the IB nodes for a given configuration of the thin structure mid-surface we begin by checking the intersection between the lines connecting the centers of fluid cells in the vicinity of the body (dashed lines) and the surface (solid lines) as shown in Fig. 2b. In three dimensions the intersection is found by checking six surrounding grid lines from the local grid point $A(i, j, k)$ and the adjacent points $B(i \pm 1, j \pm 1, k \pm 1)$ (Fig. 2b). Our searching algorithm proceeds as follows:

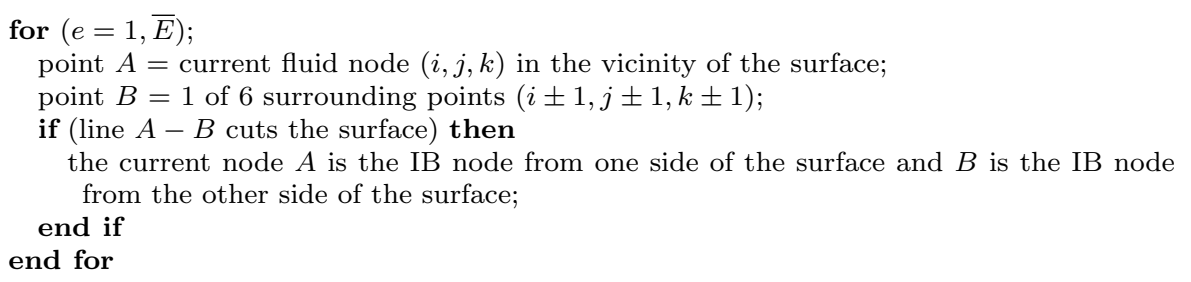

A fluid node is also considered as an IB node if the distance between the point and the center of an element $e$ is less than one grid cell size $\Delta h$, i.e. $\left|\Delta \mathbf{r}_{i b, e}\right|<\min \Delta h$, where $\Delta \mathbf{r}_{i b, e}=\left(\mathbf{r}_{i b}-\mathbf{r}_{e}\right), \mathbf{r}_{i b}$ is the position of the IB node and $\mathbf{r}_{e}$ is the position vector of the center of the triangular element (see Fig $2 \mathrm{~b}$ ). The IB node is assigned to correspond to the surface element $e$ on the solid surface.

In our parallel implementation, we utilize the bounding-box search approach [27] in order to reduce the involvement of unnecessary surface triangles in the searching algorithm. We cover the entire structure with a volume of size $\left[x_{\min }-x_{\max } ; y_{\min }-y_{\max } ; z_{\min }-z_{\max }\right]$. This volume is further divided into $N_{i} \times N_{j} \times N_{k}$ smaller bounding boxes uniformly. The choice of $N_{i}, N_{j}, N_{k}$ depends on the number of triangulated elements and the number of grid points per processors. In our simulations, $N_{i}, N_{j}, N_{k}$ are typically chosen to be less than 50. Our line intersection strategy above is applied only for fluid points and triangulated elements that belong to the same bounding box or adjacent ones.

To handle the aforementioned difficulty arising in our thin body approach, due to the fact that both positive and negative wall normal vectors at every element point toward the fluid, we separate the IB nodes in two categories: i) positive $I B^{+}$and ii) negative $I B^{-}$nodes. Note that this type of separation is crucial for the load calculation discussed in section 3.4. To determine whether an IB node is on the positive $(+)$ or negative $(-)$ side of the surface, we compute the dot product of the positive local normal vector with 
the vector connecting the center of the triangular surface element with the closest IB node: if $\left(\mathbf{n}_{e}^{+} \cdot \Delta \mathbf{r}_{i b, e}\right)>0$ then the IB node is located on the positive side, otherwise it is on the negative side (see Fig $2 \mathrm{~b}$ ). We also note that the relationship between an IB node and the corresponding surface element $e$ is not unique as there could be several IB nodes that correspond to the same solid surface element. This is particularly true when the background fluid grid size is much smaller than the triangulated cell of the solid surface. In the subsequent section we discuss how we handle surface elements that do not have a unique IB node associated with them insofar as the calculation of the forces acting on that element is concerned.

For Reynolds numbers for which the grid spacing is sufficiently fine to resolve the near wall flow, velocity boundary conditions are reconstructed at the IB nodes using the interpolation scheme along the local normal of the solid surface [39]. In our fractional step method for solving the Eq. (1) [26], this relationship is implicitly incorporated into the non-linear momentum equation and is enforced at all times. For high Reynolds number simulations a wall model is used to reconstruct boundary conditions at the IB nodes as described in [29].

\subsection{Calculation of loads on the interface $\Gamma^{\text {fsi }}$}

To enable the coupling of the fluid and structural domains in our FSI algorithm, the flow imparted loads must be calculated on the solid surface in order to provide boundary conditions for the structural solver.

A major challenge in load calculation is the need to efficiently calculate the traction vector $\boldsymbol{t}_{f}$ on the fluid-solid interface in parallel environment since the solid body can span across partitioned computational domains, which are assigned to different processors. It is thus necessary to develop a scalable algorithm to collectively compute the local loading at each processor and assemble all the information to have a complete loading distribution $\boldsymbol{t}_{f}$ on the interface $\Gamma^{f s i}$. Here we utilize the layer of the IB nodes discussed in section 3.3 above and illustrated in Figure 3. We calculate separately the pressure and viscous forces on this layer of IB nodes for each processor and the final loading condition on the IB nodes is assembled from all portions of all processors.

Since the fractional step method we employ only requires velocity boundary conditions at the IB nodes [26], the pressure $p$ at these nodes is not available. For that we calculate pressure at the IB nodes using interpolation along the normal direction in the similar fashion for the velocity components as reported in [25].

We note that we fully retain the sharp interface nature of our method in the calculation of the traction vectors even though the thin body is represented by its mid-surface. This is accomplished by using the previously discussed positive and negative wall normal vectors to independently calculate and store the forces acting on the $(+)$ and $(-)$ sides of each element on the interface using one-sided interpolation directed from the element toward 
the respective $(+)$ or $(-)$ side of the fluid nodes. Consequently, the so-calculated + and - traction vectors at each surface element exhibit a discontinuity across the thin body, which is an important physical feature of the problem preserved by this approach. The shear stress tensor components $\tau_{f, i j}$ are evaluated locally at every fluid node using the second-order differencing to compute the velocity gradients:

$$
\tau_{f, i j}=\mu\left(\frac{\partial v_{i}}{\partial x_{j}}+\frac{\partial v_{j}}{\partial x_{i}}\right) .
$$

Depending on the grid resolution, the components of the shear stress tensor $\underline{\underline{\boldsymbol{\tau}_{f}}}$ are interpolated along the normal direction $\mathbf{n}^{ \pm}$in similar fashion as the pressure [25] or reconstructed using a wall model [33] to obtain values at the $I B^{ \pm}$nodes ${\underline{\underline{\boldsymbol{\tau}_{i b}}}}^{ \pm}$. Finally, the fluid stress tensor ${\underline{\underline{\boldsymbol{\sigma}_{i b}}}}^{ \pm}$at the $I B^{ \pm}$nodes is evaluated as follows:

$$
{\underline{\underline{\boldsymbol{\sigma}_{i b}}}}^{ \pm}=-p_{i b}^{ \pm} \underline{\underline{\boldsymbol{I}}}+{\underline{\underline{\boldsymbol{\tau}_{i b}}}}^{ \pm}
$$

where $\underline{\underline{\boldsymbol{I}}}$ is the unit tensor. After the fluid stress tensor ${\underline{\boldsymbol{\sigma}_{i b}}}^{ \pm}$has been obtained, a one-sided projection procedure, from the corresponding + or $-\overline{\overline{\mathrm{IB}}}$ nodes to the actual solid surface is required to find the stress tensor on $\Gamma^{f s i}$. This procedure is described as follows. We already mentioned above in the section 3.3 that the relationship between IB nodes and a surface element $e$ is not unique as there could be several IB nodes (say $\bar{N}_{e}^{ \pm}$such nodes exist) that are associated with the same surface element $e$. Note that only either positive or negative IB nodes are involved in the load calculation process of positive or negative stresses, correspondingly. Therefore, for such cases and in order to calculate the fluid stress tensor ${\underline{\underline{\boldsymbol{\sigma}_{e}}}}^{ \pm}$on the surface of the body $\Gamma^{f s i}$ an interpolation procedure is implemented from the surrounding $I B^{ \pm}$nodes to the solid surface element as follows:

$$
\underline{\underline{\boldsymbol{\sigma}_{e}}}=\frac{\sum_{i b=1}^{\bar{N}_{e}^{ \pm}}{\underline{\underline{\boldsymbol{\sigma}_{i b}}}}^{ \pm} /\left|\Delta \mathbf{r}_{i b, e}^{ \pm}\right|}{\sum_{i b=1}^{\bar{N}_{e}^{ \pm}} 1 /\left|\Delta \mathbf{r}_{i b, e}^{ \pm}\right|}
$$

Finally, the net loading at each triangular element on the thin structure mid-surface is defined as the sum of loads from both sides of the middle surface $\boldsymbol{t}_{e}=\boldsymbol{t}_{e}^{+}+\boldsymbol{t}_{e}^{-}$, where $\boldsymbol{t}_{e}^{ \pm}=\underline{\underline{\boldsymbol{\sigma}_{e}^{ \pm}}} \cdot \boldsymbol{n}^{ \pm}{ }_{e}$ (see Fig. 4). Note that the total traction vector of the load $\boldsymbol{t}_{e}$ is calculated using $\overline{\overline{\overline{a l l}}}$ (positive and negative) IB nodes associated with the center of the triangular element $e$. In our FE solver, however, the traction vector is required at the vertices of the triangular elements $\mathbf{t}_{v}$. Thus an interpolation procedure is implemented to transfer the traction vector from the elements to the nodes using distance weighted average: $\boldsymbol{t}_{v}=$ $\boldsymbol{I}\left(\boldsymbol{t}_{e}\right)=\sum_{e=1}^{\bar{e}}\left(\boldsymbol{t}_{e} /\left|\Delta \mathbf{r}_{v, e}\right|\right) / \sum_{e=1}^{\bar{e}}\left(1 /\left|\Delta \mathbf{r}_{v, e}\right|\right)$, where the summation is implemented over all elements $\bar{e}$ adjacent to the vertex $v$ and $\left|\Delta \mathbf{r}_{v, e}\right|$ is a distance from the vertex $v$ to the center of the element $e$. 
Assuming that the structure configuration is known, the overall algorithm for calculating the flow-imparted loads on the structural surface in parallel computing environment can be summarized as follows:

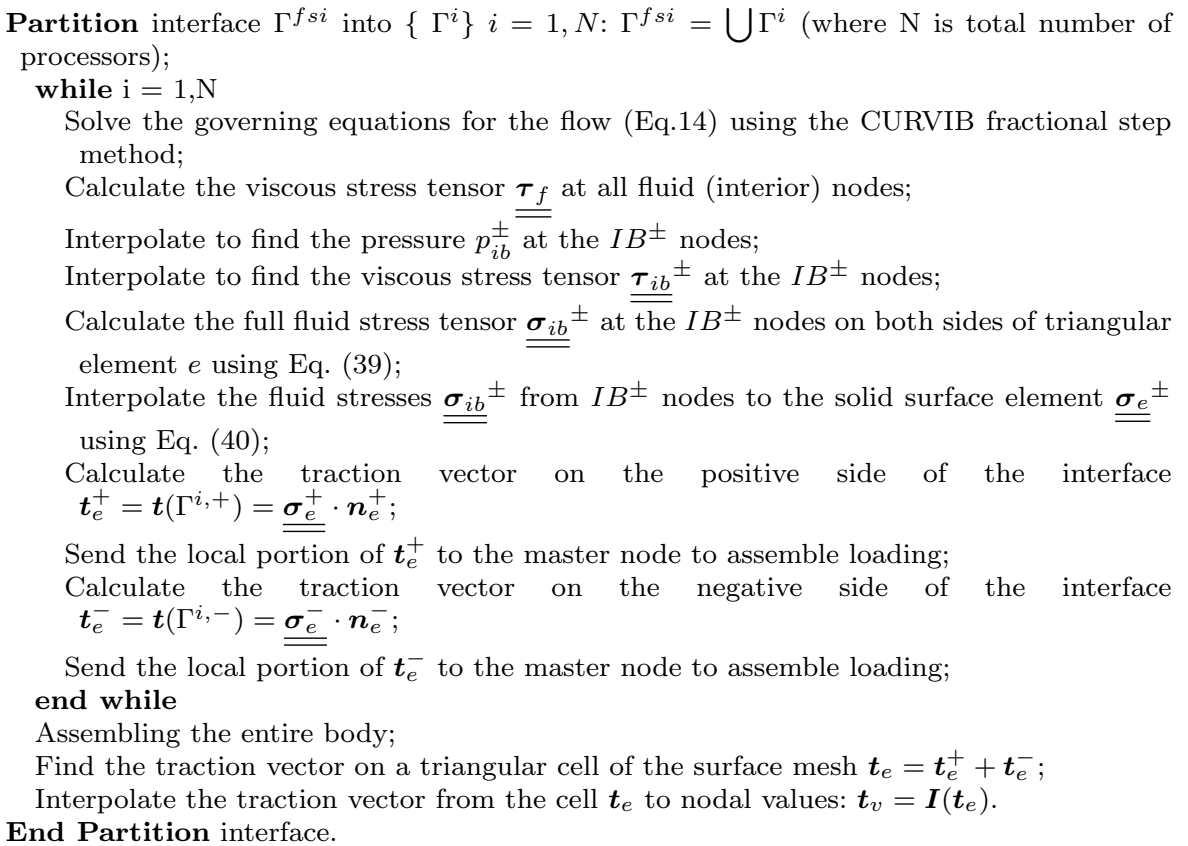

Following the implementation of this procedure the load vectors (traction vector $\boldsymbol{t}_{v}$ ) at all nodes of the $\Gamma^{f s i}$ surfaces have been defined.

\subsection{The algorithm for coupling the fluid $\mathfrak{F}$ and solid $\mathfrak{S}$ solvers}

The governing equations of the fluid (Eq.1) and solid (Eq.10) domains as well as the continuity conditions on the interface constitute a modularly partitioned fluid-structure interaction problem, which can be solved by coupling together two independent solvers: the fluid solver $\mathfrak{F}$ and the solid solver $\mathfrak{S}$. We use the conventional Dirichlet - Neumann partition [40] to couple the system of fluid-solid equations. This means that the fluid equations are solved by enforcing Dirichlet boundary condition, while the solid equations are solved by prescribing the load on the interface $\Gamma^{f s i}$ [41]. The need to enforce the continuity of the velocity and the normal stresses on the interface $\Gamma^{f s i}$ requires that both the displacement and velocity of the solid body $\mathbf{u}$ and $\dot{\mathbf{u}}$ must be tracked. We define $\mathbf{Q}$ as the solution of solid solver $\mathfrak{S}$ (Eq. 13):

$$
\boldsymbol{Q}=(\mathbf{u}, \dot{\mathbf{u}})
$$

From Eqs. (5) and (13), the FSI coupling can be formulated as a fixed point operator for 
$Q$ :

$$
\boldsymbol{Q}=\mathfrak{S} \circ \mathfrak{F}(\boldsymbol{Q})
$$

To facilitate the description of the FSI algorithm, let us assume, without loss of generality, that the pressure and velocity fields $\mathbf{v}^{n}, p^{n}$ for the fluid alongside with the displacements and velocities of the solid structure $\mathbf{u}^{n}, \dot{\mathbf{u}}^{n}$ are known at time step $n$. The fluid and structural equations, Eqn. (43)-(44), are solved to obtain the structural displacement and velocity as well as the fluid pressure and velocity fields at time-step $n+1$ with the current boundary conditions on $\Gamma^{D} \cup \Gamma^{N} \cup \Gamma^{f s i}$ via a series of sub-iterations $(l)$ to satisfy equation (42). We seek the solution of the discrete fluid operator $\mathfrak{F}$ at time step $t^{n+1}$ as:

$$
\mathfrak{F}\left(\mathbf{v}^{n+1}, p^{n+1}, \mathbf{u}^{n+1}, \dot{\mathbf{u}}^{n+1}\right)=0, \quad \text { in } \quad \Omega_{f},
$$

and the solution of the discrete solid operator $\mathfrak{S}$ as follows:

$$
\mathfrak{S}\left(\mathbf{u}^{n+1}, \dot{\mathbf{u}}^{n+1}, \boldsymbol{t}^{n+1}\right)=0, \quad \text { in } \Omega_{s} .
$$

where $\boldsymbol{t}^{n+1}=\mathfrak{T}\left(\mathbf{v}^{n+1}, p^{n+1}\right)$ is the traction vector imparted by the fluid on the body surface $\Gamma^{f s i}$. The function $\mathfrak{T}\left(\mathbf{v}^{n+1}, p^{n+1}\right)$ represents the loading on the structure surface from the pressure and velocity fields in fluid. The approach we employ to calculate $\mathfrak{T}$ in the discrete space is described in section 3.4. The fixed-point sub-iteration procedure to find $\boldsymbol{Q}$ at time steps $\mathrm{n}+1$ can thus be written as follows:

$$
\boldsymbol{Q}_{l+1}^{n+1}=\mathfrak{S}^{\mathfrak{n}+1} \circ \mathfrak{F}^{\mathfrak{n}+1}\left(\boldsymbol{Q}_{l}^{n+1}\right)
$$

where $l+1$ is the new iterate of $\mathbf{Q}^{n+1}$. In our fixed-point iteration, the fluid solver $\mathfrak{F}$ uses displacements and velocity of the solid structure $\mathbf{u}_{l}^{n+1}, \dot{\mathbf{u}}_{l}^{n+1}$ and gives new fluid velocity $\mathbf{v}_{l+1}^{n+1}$ and pressure field $p_{l+1}^{n+1}$ by solving the equations (43). The solid solver in turn uses the so updated fluid velocities, and pressure field to advance the solution of displacements and velocity of the solid structure $\mathbf{u}_{l+1}^{n+1}, \dot{\mathbf{u}}_{l+1}^{n+1}$. Sub-iterations $(l)$ are implemented every time step to satisfy the coupled system of equations and advance the solution to time step $n+1$ :

$$
\begin{aligned}
\left(\mathbf{v}_{l+1}^{n+1}, p_{l+1}^{n+1}\right) & =\mathfrak{F}\left(\mathbf{v}_{l}^{n+1}, p_{l}^{n+1}, \mathbf{u}_{l}^{n+1}, \dot{\mathbf{u}}_{l}^{n+1}\right), \\
\left(\mathbf{u}_{i+1}^{n+1}, \dot{\mathbf{u}}_{i+1}^{n+1}\right)_{l+1} & =\mathfrak{S}\left(\left(\mathbf{u}_{i}^{n+1}, \dot{\mathbf{u}}_{i}^{n+1}\right)_{l}, \boldsymbol{t}_{l}^{n+1}\right), \quad l=0,1,2, \ldots ; i=1,2,3 \ldots
\end{aligned}
$$

where index $l$ is the number of fixed point iteration and all variables at level $l=0$ are at the previous time step $\mathrm{n}$, index $i$ is the number of Newton iteration for structural equations. The sub-iterations continue until an appropriate norm of the error of the flow and structural variables between levels $l+1$ and $l$ has been reduced to a desired tolerance and the above equations have been satisfied at level $n+1$. The above procedure is generally described as a strongly-coupled FSI algorithm and ensures that the continuity of the stress 
at the fluid-structure interface is satisfied within the desired convergence threshold. If we just apply the above algorithm for one sub-iteration $(l=0)$, the requirement for the continuity of the stress is enforced only within an error that depends on the accuracy of the temporal discretization scheme. Such an algorithm is generally far more efficient than the strongly coupled approach and is referred to as loosely coupled iteration. Generally loosely coupled FSI schemes are robust for problems involving large mass ratio (structural density considerably larger than the fluid density) while strongly-coupled iterations are required to enhance robustness for problems with mass ratios of order one or lower $[1,2]$.

For mass ratio problems of order one $\left(\rho_{f} / \rho_{s} \approx 1\right)$, which arise in simulations of heart valves, the Aitken non-linear relaxation technique is also implemented to accelerate the convergence of the strongly coupled FSI algorithm [27, 42]. In summary, the overall strongly coupled FSI algorithm can be formulated as follows:

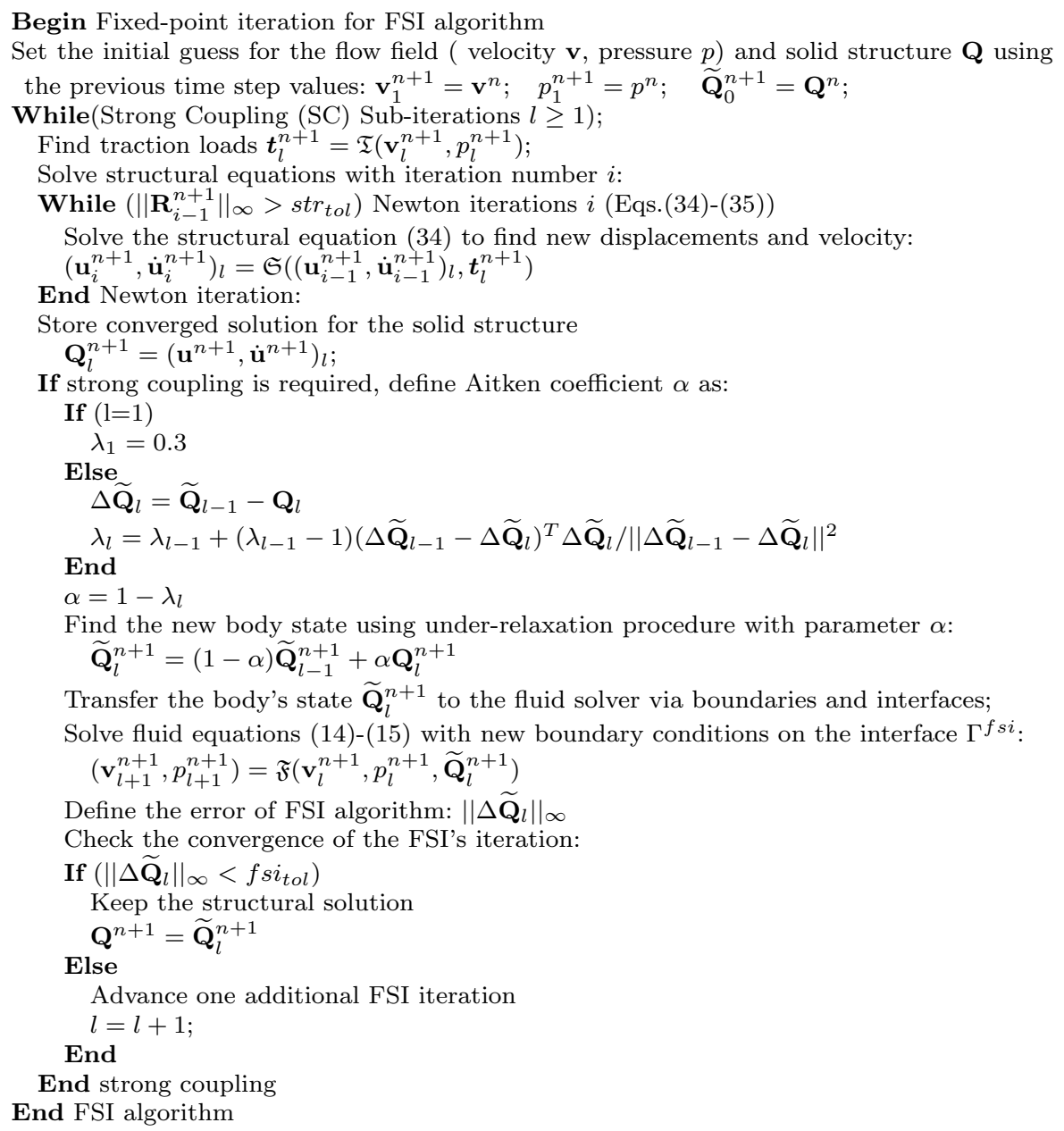

The convergence tolerance for the structural $\left(s t r_{t o l}\right)$ and strongly coupled FSI $\left(f s i_{t o l}\right)$ solvers in the above algorithm is of order $10^{-8}$ in terms of the $L_{\infty}$ norm of the $\mathbf{R}$ and $\mathbf{Q}$. Note that, we enforce the convergence of both the displacement and velocity fields of the 
structure $\mathbf{Q}$ and thus we employ a strict requirement for convergence.

We note that for all the cases we simulate in this work, although the underlying FSI dynamics of complex, the degrees of freedom (DOF) for the structural mesh are quite low (in the order of thousands) compared to the fluid DOFs (in the order of millions) since the structures are relatively simple. Thus the cost for solving the solid equations is small in comparison to the fluid equations. For that the solid solver is implemented as a serial code possessed by the root processor. All processors involved in the solution of the fluid equations receive the same image $\mathbf{Q}$ of the structure from the root processor.

Our code is parallelized and uses the Petsc Library. The simulations we report herein have been carried out on a cluster with dual 8-core AMD 6112. To estimate the efficiency of the code we report the CPU time per node of the computational grid, per processor and per time step. For the inverted flag problem we report in section 4.2 below this quantity is equal to $t_{C P U} /($ Nodes $\cdot$ Procs $\cdot$ ntime $) \approx 3 \cdot 10^{-2} \mu s$.

\section{Results and discussion}

In this section we demonstrate the predictive capabilities of the proposed CURVIB-FEFSI algorithm by applying it to simulate several problems of increasing complexity, all involving FSI with thin flexible structures. We first validate the method by presenting simulation results for two cases for which benchmark numerical or experimental data are available: 1) The vortex induced vibrations of a cantilever mounted in the wake of a square cylinder, which has been studied numerically by several authors, including, among others, [43, 44, 45]; and 2) The vibrations of an inverted flexible flag, which has been studied experimentally by Kim et al. [32]. Subsequently we demonstrate the ability of the CURVIB-FE-FSI algorithm to simulate pulsatile, physiologic flow through a tri-leaflet aortic valve placed in an anatomic aorta. This is more challenging problem compared to the two validation cases, because it is geometrically more complex, is characterized by low mass ratio $\left(\rho_{s} / \rho_{f} \sim 1\right)$ and imposes a more stringent overall test for the stability and robustness of the FSI solver.

\subsection{Vortex induced vibration of a cantilever}

We consider a 2D cantilever mounted at the downstream face of a 2D square cylinder as shown in Figure 5. Depending of the flow regime, which is determined by the Reynolds number based on the inflow velocity $u_{\infty}$ and cylinder size $L_{0}$, and the geometric (cantilever length $L$ and thickness $h_{0}$ ), material (structure density $\rho_{s}$ ) and structural (Young modulus $E$, Poison ratio $\nu$ ) properties of the cantilever, the cantilever may develop complex vibration modes. More specifically, vibrations with frequency and kinematics close to either the first or the second natural modes of the cantilever may be excited in such a 
configuration [45]. In this section we present results for two cases, corresponding to these distinct vibration states of the structure.

The input data for the first problem we solve correspond to the problem solved by Wall and Ramm [43] and gives rise to cantilever vibrations at a frequency close to the first natural frequency (the so-called first natural mode of cantilever vibrations). The kinematics of this mode is characterized by vibration amplitudes that increase monotonically as the downstream free tip of the structure is approached. In the simulation we report herein we employ the following properties for the structure and the fluid: shell's density $\rho_{s}=$ $10^{2} \mathrm{~kg} / \mathrm{m}^{3}$, Young's modulus $E=2.5 \cdot 10^{5} \mathrm{~Pa}$, Poisson's ratio $\nu=0.35$, shell's thickness $h_{0}=6 \cdot 10^{-4} \mathrm{~m}$, cantilever length $L=4 \cdot 10^{-2} \mathrm{~m}$, fluid density $\rho_{f}=1.18 \mathrm{~kg} / \mathrm{m}^{3}$, fluid viscosity $\mu=1.82 \cdot 10^{-5} \mathrm{~Pa} \mathrm{~s}$, inflow velocity $u_{\infty}=0.513 \mathrm{~m} / \mathrm{s}$. The length $L_{0}=10^{-2} \mathrm{~m}$ of the square cylinder is taken as a characteristic length yielding a Reynolds number of the flow $R e=333$; length of the cantilever is equal to $L=0.04 \mathrm{~m}$. Even though the problem is $2 \mathrm{D}$ we solve it as $3 \mathrm{D}$ with symmetry boundary conditions in the transverse direction. The cantilever is discretized with 320 triangular elements and the background fluid grid is discretized with a mesh with 161x91x5 in the streamwise (x), vertical (y) and transverse (z) directions, respectively. The background mesh is stretched both in the streamwise and vertical directions in the vicinity of the cantilever (see Fig. 5). The minimum grid spacing in the vicinity of the square cylinder and the cantilever is $h_{0}=6.45 \cdot 10^{-4} \mathrm{~m}$, which means that there are 62 fluid grid nodes along the length of the cantilever. The nondimentional time step is equal to $\Delta \widetilde{t}=0.005$. This problem is characterized by moderate mass ratio of density $\left(\rho_{s} / \rho_{f} \sim 85\right)$ and in order to get converged solution requires to implement strong coupling procedure described above. Typically 3-4 strong coupling iterations are required to reduce the residual by 8 orders of magnitude each time step.

In Fig. 6 we plot the calculated time histories of (a) the trajectory of the tip displacement and (b) the cantilever tip velocity. It is evident that following an initial transient stage, constant amplitude, periodic oscillations are established for both quantities. The corresponding flow patterns, cantilever shape and location are shown in Figure 7, which depicts contours of instantaneous out of plane vorticity field. As evident from this figure, cantilever vibrations are excited by the vortices shed by the square cylinder. The cantilever kinematics is consistent with the first natural mode of the structure [43, 46, 11, 47] with the displacement increasing monotonically along the cantilever length and becoming maximum at the free tip.

To demonstrate that our CURVIB-FE-FSI method can yield grid converged solutions, we carry out a grid refinement study for the first mode of cantilever vibrations. Five successively finer grids are used for the convergence study, denoted as: grid 1 with $81 \times$ $45 \times 5$ nodes; grid 2 with $161 \times 91 \times 5$ nodes; grid 3 with $321 \times 181 \times 5$ nodes; grid 4 with $641 \times 365 \times 5$ nodes and grid 5 with $1281 \times 721 \times 5$ nodes. The cantilever is discretized with 320 elements. The nondimentional time step for all grids is equal to $\Delta \widetilde{t}=0.005$. For grids 1 to 5 we calculate the frequency of cantilever oscillations $f_{i}$ as follows: $f_{1}=3.8 \mathrm{~Hz}, f_{2}=3.5 \mathrm{~Hz}, f_{3}=3.31 \mathrm{~Hz}, f_{4}=3.24 \mathrm{~Hz}$ and $f_{5}=3.2 \mathrm{~Hz}$. The 1 st natural frequency of the cantilever, which one can get from an analytical formulation [48], 
and the frequency of vortex shedding from the square cylinder, which we obtain from numerical simulations of flow past the square cylinder without the cantilever, are equal to $f_{1}^{\text {cant }}=3.033 \mathrm{~Hz}$ and $f_{1}^{\text {fluid }}=6.65 \mathrm{~Hz}$, respectively. It is well known from the theory of structural vibrations that when the motion of the structure is excited by an external force with a forcing frequency $f_{\text {force }}$, the structure will oscillate with the same frequency as that of the imposed force. In FSI problems, on the other hand, the frequency of the cantilever will increase compared to its natural frequency $f_{1}^{c a n t}$ [45]. The results of the grid refinement study are shown in Fig. 8 and clearly show that as the grid is refined the calculated frequency converges monotonically toward $f_{e}=3.2 \mathrm{~Hz}$.

In Table 1 we compare our simulated results with previously published results in the literature. It is evident from the data in this table that in spite of differences between the numerical methods used in all these studies, our results are in a very good agreement with previously published data $[43,46,11,47]$ both in terms of the calculated frequency of oscillations and tip deflection amplitude.

\begin{tabular}{|l|l|l|}
\hline Author & $\begin{array}{l}\text { Frequency } \\
(\mathrm{Hz})\end{array}$ & $\begin{array}{l}\text { Tip } \\
\text { deflection }(\mathrm{cm})\end{array}$ \\
\hline Wall and Ramm, (1998) [43] & $2.77-3.22$ & $1.12-1.32$ \\
Matthies and Steindorf, (2003) [46] & 3.125 & $1.00-1.35$ \\
Dettmer and Perić, (2006)[11] & $2.94-3.125$ & $1.00-1.40$ \\
Wood et al., (2008) [47] & $2.94-3.125$ & $1.10-1.20$ \\
Current work & 3.2 & $1.00-1.10$ \\
\hline
\end{tabular}

Table 1. Comparison of our computational data for the period of oscillation and maximum value of the tip displacement with data published in [43, 46, 11, 47].

The second cantilever problem we solve is identical to the problem solved by Hübner et al. [44] and gives rise to the second natural mode of cantilever vibrations, namely kinematics resembling a travelling wave with a stagnation point. The following values for the various parameters are used for this problem: shell's density $\rho_{s}=2 \cdot 10^{3} \mathrm{~kg} / \mathrm{m}^{3}$, Young's modulus $E=2 \cdot 10^{5} \mathrm{~Pa}$, Poisson's ratio $\nu=0.35$, shell's thickness $h_{0}=6 \cdot 10^{-4} \mathrm{~m}$, fluid density $\rho_{f}=1.18 \mathrm{~kg} / \mathrm{m}^{3}$, fluid viscosity $\mu=1.82 \cdot 10^{-5} \mathrm{Ps}$ s, inflow velocity $u_{\infty}=0.315 \mathrm{~m} / \mathrm{s}$. The square cylinder length is $L_{0}=10^{-2} \mathrm{~m}$ and the resulting Reynolds number is $R e=204$. The cantilever is discretized with 320 triangular elements and the background fluid grid is discretized with a mesh $161 \times 91 \times 5$, the nondimentional time step is equal to $\Delta \widetilde{t}=0.01$. From the previously reported grid sensitivity study for the 1st mode of vibrations, it follows that this grid resolution is sufficiently fine to predict the frequency of vibrations within less than 10 percent of the grid converged value. This problem is characterized by high density ratio $\left(\rho_{s} / \rho_{f} \sim 1690\right)$, hence the loose coupling FSI iteration scheme is adequate for obtaining robust solutions. In Fig. 9 we plot the calculated time histories of the trajectory of the tip displacement and the cantilever tip velocity to demonstrate that our simulations converge to a statistically stationary state for the second mode of oscillations. 
In contrast to the previous case with the first mode oscillation, the second mode requires much longer times to reach the statistically stationary state for the displacement and velocity. This should be attributed to the higher shell density for this case and the associated dynamics due to inertia. As shown in Fig. 10, our simulations capture the distinctly different mode of vibrations, than those shown in Fig. 6, with the cantilever kinematics corresponding to the propagation of a travelling wave with a fixed point. This is clearly seen in Fig. 11 where comparisons of instantaneous cantilever shapes for the first (a) and the second (b) mode of oscillation are shown. The calculated amplitude and frequency of the vibrations for this second mode of cantilever oscillations are equal to $y_{\max } \simeq 7.5 \cdot 10^{-3} \mathrm{~m}$, $f \simeq 3.4 \mathrm{~Hz}$, respectively, and they are very close to those reported by Hübner et al. [44] $y_{\max } \simeq 8.0 \cdot 10^{-3} \mathrm{~m}, f \simeq 3.1 \mathrm{~Hz}$ and by Valdes [45] $y_{\max } \simeq 8.0 \cdot 10^{-3} \mathrm{~m}, f \simeq 3.22 \mathrm{~Hz}$.

\subsection{Oscillations of a flapping inverted flag}

The second validation test case is a more stringent and challenging case as it involves the flapping oscillations of an inverted flag at high Reynolds number and corresponds to a recently published laboratory experiment [32]. The problem is referred to as the inverted flag because the flag, a thin flexible sheet of length $\mathrm{L}$, is mounted on its trailing edge with its leading edge free to move in response to a uniform incoming flow $u_{\infty}$ (see Fig. 12). Kim et al. [32] carried out a series of experiments by varying $u_{\infty}$ and/or the structural properties of the flag and identified a dynamically rich phase space of flag responses. They showed that the non-dimensional parameter that governs the dynamics of the FSI problem is the nondimensional bending stiffness $\beta$ :

$$
\beta=\frac{B}{\rho_{f} u_{\infty}^{2} L^{3}},
$$

where $B$ is a flexural rigidity $B=E h_{0}^{3} / 12\left(1-\nu^{2}\right)$ of the flag, $\rho_{f}$ is the fluid density, and $h_{0}$ is the thickness of the plate. Kim et al. [32] identified three regimes of flag response as a function of $\beta: 1$ ) the straight mode, where the flag is too rigid to be deflected by the flow and remains straight (large values of $\beta$ ); 2) the flapping mode, where the flag undergoes large amplitude flapping oscillations (intermediate values of $\beta$ ); and 3 ) the deflected mode, where the flag is so flexible that it is deflected by the flow toward one side and remains fixed at this position at all times (small $\beta$ values). Here we report simulations for $\beta=0.1$, which is in the flapping regime. This regime is quite challenging from the FSI simulation standpoint as it involves very large amplitude oscillations. The specific $\beta$ value is selected because for this value the experiment of [32] revealed a complex dynamic response of the flag characterized by rich flapping dynamics including several local minima and maxima of the flag leading edge position during the cycle of flapping motion. We carry out simulations for the following values of the various governing parameters for this problem: $E=2.38 \cdot 10^{9} \mathrm{~Pa}, \nu=0.38, \rho_{s}=1.2 \cdot 10^{3} \mathrm{~kg} / \mathrm{m}^{3}, h_{0}=8 \cdot 10^{-4} \mathrm{~m}, H=L=0.3 \mathrm{~m}$, $u_{\infty}=6.7 \mathrm{~m} / \mathrm{s}, \mu=1.92 \cdot 10^{-5} \mathrm{~Pa} \mathrm{~s}, \rho_{f}=0.98 \mathrm{~kg} / \mathrm{m}^{3}$, hence $B=0.118 \mathrm{Nm}$ and $\beta=0.1$. The corresponding Reynolds number, based on the inflow velocity and flag length, is 
$R e=u_{\infty} \rho_{f} L / \mu=99505$, and, therefore, the massively separated flow in the wake of the flapping flag is expected to be turbulent. For that we employ the CURVIB-FE-FSI method in LES mode with three-point central differencing for the convective terms in the flow equations, the dynamic Smagorinsky subgrid scale model [34] for closure, and the wall model of [49] to reconstruct boundary conditions on the flag as adapted for the CURVIB method by Kang et al. [33,30]. The plate surface is discretized with 206 triangle elements and the background fluid grid is discretized with a uniform Cartesian mesh with $561 \times 201 \times 201$ in the streamwise $(\mathrm{x})$, vertical $(\mathrm{y})$ and transverse $(\mathrm{z})$ directions, respectively. The nondimentional time step is equal to $\Delta \widetilde{t}=0.01$.

Fig. 13 compares the measured [32] and computed time histories of the flag leading edge deflection. It is seen that the computed results are in excellent agreement with the experimental measurements. The simulations not only capture the amplitude and period of oscillations with good accuracy but also resolve the two local deflection maxima (minima) that occur in the vicinity of maximum (minimum) flag deflection. A more quantitative comparison with the measurements reveals that the maximum discrepancy between experiments and simulations, which occurs around maximum and minimum tip deflection, does not exceed $7 \%$ of the measured values.

To demonstrate the sensitivity of the solutions we obtain for this problem to grid refinement, we construct three successively coarser grids than the fine mesh we discussed above. That is we carry out simulation on a total of four meshes with the following number of grid nodes: grid 1 with $141 \times 51 \times 51$ nodes; grid 2 with $201 \times 71 \times 71$ nodes; grid 3 with $281 \times 101 \times 101$ nodes and grid 4 with $561 \times 201 \times 201$ nodes. The flag surface is discretized with 206 triangle elements, the nondimentional time step is equal to $\Delta t=0.01$. In Fig. 14 we demonstrate the convergence of the calculated flag Strouhal number (St) as the grid is refined from grid 1 to 4 . The dashed line in this figure indicates the experimental value of $S t \simeq 0.08$ which is reported in Fig. $2 \mathrm{~b}$ of [32]. It is evident from this figure that the computed Strouhal number converges monotonically as the mesh is refined and that grid 4 yields results in excellent agreement with the measurements. In Fig. 15 the trajectory of the flag tip during one cycle is obtained on the four successively finer grids. Once again, the computed solution converges monotonically toward a grid converged solution as the grid is refined. All subsequently shown results are presented on the finest grid, grid 4.

In Fig. 16 we plot the time history of drag, lift, and span-wise force coefficients. Experimentally measured maximum drag and mean drag coefficients for $\beta=0.1$ are equal to $C_{D, \text { max }} \approx 2$ and $C_{D, \text { mean }} \approx 1$ respectively, (see [32], Fig.5a). The computed drag coefficients $C_{D, \text { max }}^{\text {comput }} \approx 2$ and $C_{D, \text { mean }}^{\text {compute }} \approx 1$ and are in excellent agreement with experimental values. The small amplitude, albeit clearly evident from Fig. 16, temporal variation of the spanwise force coefficient suggests that the flag develops complex motion that includes both up and down flapping and twisting modes. To further illustrate this feature of the flag motion, which would be difficult to document experimentally, we plot in Fig. 17 the three-dimensional shapes of the flag at various instants within one half period of the oscillations. The flapping and twisting modes that develop are clearly evident from this figure. 
Fig. 18 depicts the calculated instantaneous out of plane vorticity field at various instants during the flapping cycle. These snapshots as well as video animations of the vorticity field, not shown herein, clearly show that the flapping dynamics is correlated with the formation of a large leading edge vortex as the flag tip approaches maximum deflection. The vortex begins to form as the flag moves upward (or downward) as a result of shearlayer roll up and leads to massive separation and shedding of vorticity in the wake at maximum (minimum) deflection. The resulting wake is very complex and exhibits a largescale meandering motion as a result of the continuous flapping motion of the flag. The computed results shown in Fig. 18 are in good overall qualitative agreement with the flow visualizations reported by Kim et al. [32] and their overall description of the underlying wake dynamics as obtained in their experiments.

To elucidate the three-dimensional structure of this highly unsteady and massively separated wake, we plot in Fig. 19 several snapshots of the Q-criterion [50]. It is evident from this figure that the flow is dominated by shear-layer roll-up off the sharp edges of the flag, which leads to the formation of an arch vortex along the leading edge and intertwined spiral vortex tubes shed off the two sides of the flag. These structures separate from the flag and break up into small scale turbulence in the wake.

To our knowledge the results we have reported herein elucidate for the first time the three-dimensional structure of the wake of a flapping inverted flag and clearly illustrate the ability of our CURVIB-FE-FSI method to solve a very complex, high Reynolds number problem involving complex large amplitude vibrations of a thin structure. Even though not shown herein, we have carried out simulations for values of $\beta$ in all three experimentally identified flag response regions and our results are in very good agreement with the experiments of [32]. These results will be reported in a separate paper currently in preparation.

\subsection{FSI simulation of tri-leaflet aortic valve}

In this section we demonstrate the ability of the method to simulate physiologic flow through a tri-leaflet aortic valve located in an anatomically realistic aorta. The flow through the aorta is driven by a prescribed physiologic flow wave form at the aorta inlet and the response of the valve leaflets and associated flow field are simulated by the new CURVIB-FE-FSI algorithm.

We consider a tri-leaflet aortic heart valve and model it as a thin shell using the rotational free FE formulation of [24] as described in section 3.2 above. We use in our simulations a model suitable for a prosthetic polymeric aortic valve with isotropic material and the Neo-Hookean constitutive equation. The geometric and material characteristics of the valve are specified from values available in the literature to correspond to a prosthetic polymeric valve [51] and are as follows: valve diameter $d_{0}=0.0254 \mathrm{~m}$, leaflet thickness $h_{0}=6.0 \cdot 10^{-4} \mathrm{~m}$, Young modulus $E=1 \mathrm{MPa}$, Poisson coefficient $\nu=0.35$, and density 
$\rho_{s}=1.2 \cdot 10^{3} \mathrm{~kg} / \mathrm{m}^{3}$. As shown in Fig. 20 the valve is placed in an anatomic aorta, which has been reconstructed from patient-specific MRI data - this is the same aorta geometry used in Le and Sotiropoulos [52] for the simulations of a mechanical bi-leaflet heart valve $(\mathrm{MBHV})$.

The pulsatile flow wave form we prescribe as inflow boundary condition at the inlet of the aorta domain is shown in Fig. 21. The corresponding heart beat is equal to $70 \mathrm{bpm}$, which gives a period of the cardiovascular cycle $T=0.857 \mathrm{~s}$. The valve diameter $d_{0}$ is used as the characteristic length scale and the peak systolic velocity of $U_{0}=0.8 \mathrm{~m} / \mathrm{s}$ is used as the velocity scale. Using the viscosity of blood $\mu=3.52 \cdot 10^{-3} \mathrm{~Pa} s$, and blood density $\rho_{f}=1050 \mathrm{~kg} / \mathrm{m}^{3}$, gives a peak systolic Reynolds number $\mathrm{Re}=6000$, which well within the physiologic range $[53,51]$. The characteristic time scale is equal to $T_{0}=d_{0} / U_{0}=3.1 \cdot 10^{-2} \mathrm{~s}$ and thus the non-dimensional period of cardiac cycle is $\widetilde{T}=T / T_{0}=0.857 / 3.1 \cdot 10^{-2}=27.6$ non-dimensional time units. The non-dimensional time step for the simulations is set equal to $\Delta \widetilde{t}=0.01$, which corresponds to discretizing the cardiac cycle with $N_{\text {time }}=$ $\widetilde{T} / \widetilde{t}=2760$ computational time steps. Since the density ratio for this problem is of order one, the strong coupling FSI iteration is required for stable and robust simulations. In all subsequently presented simulations 4-10 strong coupling iterations are sufficient to reduced the residuals by 8 orders of magnitude.

The overall computational set up is shown in Fig. 20 and consists of (a) the anatomic aorta, (b) the flexible tri-leaflet prosthetic heart valve, (c) the rigid valve support structure, and (d) housing. A curvilinear boundary-fitted grid is used to discretize aorta domain with $101 \times 101 \times 601$, in the two transverse and streamwise directions, respectively. This grid is identical to that used by Le and Sotiropoulos [52] to discretize the same aorta geometry but with a MBHV. The valve leaflets are discretized with 476 triangle elements.

The flow wave form shown in Fig. 21, which corresponds to the systolic phase of the cardiac cycle during which the aortic valve opens and closes, is used to specify time-dependent Dirichlet conditions for the velocity at the inlet. At the outlet of the aorta zero-gradient Neumann condition $\partial \mathbf{v} / \partial n=0$ is applied for all three velocity components along with a correction of the so-resulting velocity field to enforce global mass conservation. No slip and no-flux boundary conditions are applied on all solid surfaces.

We note that in our numerical method the discrete continuity equation is satisfied to machine zero at each time step, thus preserving the incompressible nature of the flow locally and globally. This is accomplished by solving the Poisson equation in the projection step of the fractional step method with the residual reaching machine zero at every physical time step. For more details we refer the reader to [26].

The calculated flowfields for one simulated systolic cardiac cycle are shown in Fig. 22. Contours of instantaneous vorticity magnitude are plotted in this figure on a plane passing through the center of the aorta. As seen in this figure, a well defined vortex ring forms as soon as the valve opens at early systole (Fig. 22a). Shear layers connecting the aortic valve vortex ring with the valve leaflets are also evident in Fig. 22a. As the valve leaflets continue 
to open, the vortex ring advances and impinges on the curved aorta wall and breaks up. The valve leaflet shear layers intensify as the flow rate through the valve increases and the flow in the wake of the valve leaflets is seen to break up into small-scale turbulence at approximately halfway within the accelerating phase of the cardiac cycle (Fig. 22c). By the time the peak systolic flow is reached and the valve has opened fully, the flow in the aorta is seen to have transitioned to a fully turbulent state downstream of the valve leaflets (Fig. 22d). This state persists even after the valve closes and the flow structures in the aorta gradually decay (Fig. 22f).

The results shown in Fig. 22 reveal significant differences between the simulated flow patterns reported in Le and Sotiropoulos [54] for a MBHV in the same anatomic aorta. More specifically, when a MBHV is implanted in the aortic position the turbulent state downstream of the valve leaflets does not emerge until shortly after peak systole. For the tri-leaflet valve, however, Fig. 22 clearly shows that the flow transitions to turbulence well before peak systole is reached. This finding should be attributed to the complex vortex dynamics induced by the shape of the tri-leaflet valve orifice as it opens and the interaction of the aortic valve vortex ring with the aorta wall. To illustrate the three-dimensional dynamics of coherent structures as the valve opens, we plot in Fig. 23 instantaneous snapshots of the Q iso-surface [50]. As seen in Fig. 23a, as the valve opens the shear layer from the valve leaflets rolls up to form a three-lobed vortex ring that follows the shape of the valve orifice. As the valve continues to open, this vortex ring becomes distorted as each one of its three lobes, forming at the valve commissures, bends forward and propagates at faster speed than the rest of the ring. This complex deformation of the aortic valve ring is clearly evident in Fig. 23c where three distinct vortex loops are seen to have formed. Each loop forms because of the faster propagation and associated stretching of the corresponding lobe of the initial ring. Essentially the vortex interactions and instabilities revealed by our simulations are similar to those observed in pulsatile flow through corrugated nozzles [55]. These instabilities along with the subsequent impingement of the three-lobed aortic valve ring on the aorta wall are ultimately responsible for the relatively early transition to turbulence of the flow in the wake of a tri-leaflet valve leaflets as compared to the considerably slower transition observed in the wake of a MBHV in the same aorta anatomy [52].

To our knowledge the results we have presented herein represent the first FSI simulation of a tri-leaflet heart valve in an anatomic aorta at physiologic conditions. The ability of the method to resolve the very complex flow patterns and associated vorticity dynamics as the valve leaflets open and close illustrates its potential as a powerful tool for patient-specific simulations of native and prosthetic heart valves.

\section{Conclusions}

We have developed a new computational approach for simulating fluid-structure interaction (FSI) problems in complex domains with thin flexible solid structures. Our method 
is based on integrating our sharp-interface CURVIB solver, previously developed for FSI problems with rigid structures [27], with an accurate and efficient rotation-free FE formulation for thin shells [24] into a coupled FSI framework that is able to handle thin structures undergoing arbitrarily large oscillation amplitudes. Two set of problems with available experimental and computational data were chosen to validate our new method: (1) the oscillations of a cantilever mounted on the wake of a square cylinder; and (2) the flapping dynamics of an inverted flag. Comparisons of our simulations and previously published simulations for cantilever problems show excellent agreement. The inverted flag case in particular, which to the best of our knowledge was simulated numerically for the first time, demonstrated the ability of our method to simulate with LES a dynamically rich, high-Reynolds number FSI problem. Comparisons with the measurements of [32] for this case revealed the ability of the method to capture even subtle features of the flag kinematics, such as the existence of multiple local extrema near the location of maximum deflection, and reproduce wake structures similar to those visualized in the laboratory. We subsequently applied our method to simulate the dynamics of a tri-leaflet aortic heart valve placed in an anatomic aorta to demonstrate the capability of the method to solve complex FSI problems in realistic cardiovascular anatomies and at physiologic conditions. Our simulations elucidated the rich vorticity dynamics during the opening of the valve leaflets, which is dominated by a distorted, three-lobed vortex ring forming as the shear layer rolls up off the valve leaflets. This vortex ring subsequently undergoes complex instabilities and breaks up into small scale turbulence before peak systole is reached. To our knowledge, the simulations we have reported herein are the first to simulate a tri-leaflet heart valve in a realistic anatomic aorta.

In this paper, a model of artificial heart valve with constant thickness and linear constitutive equations for leaflets has been used. We are currently extending the method to incorporate nonlinear constitutive equations [56] with variation of thickness to enable simulations of native aortic valves. Moreover, in our model leakage flow is allowed at the end of cardiac cycle as there exist a small gap between the leaflet tips. This leakage flow exists in many types of tri-leaflet prosthetic valves as a design feature and it is well documented in the literature [57]. We are also in the process of extending the valve model to enable full contact of the leaflets and these developments will be reported in a future paper focusing on native aortic valve simulations.

In our current approach, the solid domain is handled using a single processor. This is due to the small size of the structural mesh in the problems we have simulated in this work. Our code, however, can be readily extended to handle multiple flexible bodies submerged in a fluid domain. In such a case, multiple processors should be involved to in the solid solver. This can be accomplished by assigning a single processor to handle one flexible body. Since the solid FE solver is very efficient, the overall computational time for this type of problem will not differ from a single solid body problem. However, the computational cost of the structural domain will significantly increase if a large number of degrees of freedoms are needed for the structural mesh. To circumvent this difficulty the structural solver will need to be parallelized. A version of our CURVIB-FE-FSI solver we reported herein with fully parallelized structural solver is currently under development and will be 
presented in our future work.

\section{Acknowledgments.}

We acknowledge the financial support from a grant from the Lillehei Heart Institute at the University of Minnesota, NSF grant IIP-1318201, and U.S. Department of Energy grant (DE-EE 0005482). Computational resources have been provided by of the Minnesota Supercomputing Institute. 


\section{References}

[1] Fotis Sotiropoulos and Xiaolei Yang. Immersed boundary methods for simulating fluid - structure interaction. Progress in Aerospace Sciences, 65:1-21, 2014.

[2] Hyoungsu Baek and George Em Karniadakis. A convergence study of a new partitioned fluid-structure interaction algorithm based on fictitious mass and damping. Journal of Computational Physics, 231:629-652, 2012.

[3] C. W. Hirt, A. A. Amsden, and J. L. Cook. An Arbitrary Lagrangian-Eulerian computing method for all flow speeds. J. Comp. Phys., 14(3):227-253, 1974.

[4] J. Donea, S. Giuliani, and J.P. Halleux. An Arbitrary Lagrangian - Eulerian finite element method for transient dynamic fluid - structure interactions. Computer Methods in Applied Mechanics and Engineering, 33:689-723, 1982.

[5] R Mittal and G Iaccarino. Immersed boundary methods. Ann Rev Fluid Mech, 37:239-261, 2005.

[6] Boyce E Griffith, Xiaoyu Luo, David M McQueen, and Charles S Peskin. Simulating the fluid dynamics of natural and prosthetic heart valves using the immersed boundary method. International Journal of Applied Mechanics, 1(01):137-177, 2009.

[7] Jeffrey K Wiens and John M Stockie. An efficient parallel immersed boundary algorithm using a pseudo-compressible fluid solver. Journal of Computational Physics, 281:917-941, 2015.

[8] Luoding Zhu and Charles S Peskin. Simulation of a flapping flexible filament in a flowing soap film by the immersed boundary method. Journal of Computational Physics, 179(2):452-468, 2002.

[9] Duc-Vinh Le, J White, Jaime Peraire, KM Lim, and BC Khoo. An implicit immersed boundary method for three-dimensional fluid-membrane interactions. Journal of Computational Physics, 228(22):8427-8445, 2009.

[10] Haoxiang Luo, Rajat Mittal, Xudong Zheng, Steven A. Bielamowicz, Raymond J. Walsh, and James K. Hahn. An immersed-boundary method for flow - structure interaction in biological systems with application to phonation. Journal of Computational Physics, 227:9303-9332, 2008.

[11] W. Dettmer and D. Perić. A computational framework for fluid-structure interaction: finite element formulation and applications. Computer Methods in Applied Mechanics and Engineering, 195(41):5754-5779, 2006.

[12] Andrew T. Barker and Xiao-Chuan Cai. Scalable parallel methods for monolithic coupling in fluid - structure interaction with application to blood flow modeling. Journal of Computational Physics, 229:642 - 659, 2010.

[13] Y Bazilevs, M-C Hsu, and MA Scott. Isogeometric fluid-structure interaction analysis with emphasis on non-matching discretizations, and with application to wind turbines. Computer Methods in Applied Mechanics and Engineering, 249:28-41, 2012.

[14] X. Zheng, Q. Xue, R. Mittal, and S. Beilamowicz. A coupled sharp-interface immersed boundary-finite element method for flow-structure interaction with application to human phonation. Journal of Biomechanical Engineering, 132:111003-1 - 11100312, 2010.

[15] Charbel Farhat and Vinod K.Lakshminarayan. An ALE formulation of embedded boundary methods for tracking boundary layers in turbulent fluid - structure inter- 
action problems. Journal of Computational Physics, 263:53-70, 2014.

[16] Ge and Sotiropoulos. Direction and magnitude of blood flow shear stresses on the leaflets of aortic valves: Is there a link with valve calcification? Journal of Biomechanical Engineering, 132, 2010.

[17] Haoxiang Luo, Bo Yin, Hu Dai, and James F. Doyle. A 3d computational study of the flow - structure interaction in flapping flight. Technical report, 48th AIAA Aerospace Sciences Meeting Including the New Horizons Forum and Aerospace Exposition 4 7 January 2010, Orlando, Florida, 2010.

[18] Tian Fang-Bao, Hu Dai, Haoxiang Luo, James F.Doyle, and Bernard Rousseau. Fluid - structure interaction involving large deformations: 3d simulations and applications to biological systems. Journal of Computational Physics, 258:451-469, 2014.

[19] David Kamensky, Ming-Chen Hsu, Dominik Schillinger, John A. Evans, Ankush Aggarwal, Yuri Bazilevs, Michael S. Sacks, and Thomas J. R. Hughes. An immersogeometric variational framework for fluid.structure interaction: Application to bioprosthetic heart valves. Computer Methods in Applied Mechanics and Engineering, 284:1005-1053, 2015.

[20] Iman Borazjani. Fluid - structure interaction, immersed boundary - finite element method simulations of bio-prosthetic heart valves. Comput. Methods Appl. Mech. Engrg., 257:103-116, 2013.

[21] MS Sacks, FJ Schoen, and Jr JE Mayer. Bioengineering challenges for heart valve tissue engineering. Annual review of biomedical engineering, 11:289-313, 2009.

[22] H. Stolarski, T. Belytschko, and S.-H. Lee. A review of shell finite elements and corotational theories. Computational Mechanics Advances, 2:125-212, 1995.

[23] E. Gal and R. Levy. Geometrically nonlinear analysis of shell structures using a flat triangular shell finite element. Archives of Computational Methods in Engineering, 13:331-388, 2006.

[24] H. Stolarski, A. Gilmanov, and F. Sotiropoulos. Non-linear rotation-free 3-node shell finite-element formulation. International Journal for Numerical Methods in Engineering; 2013., 95:740-770, 2013.

[25] A. Gilmanov and F. Sotiropoulos. A hybrid cartesian/immersed boundary method for simulating flows with 3d, geometrically complex, moving bodies. Journal of Computational Physics, 207(2):457, 2005.

[26] L. Ge and F. Sotiropoulos. A numerical method for solving the 3d unsteady incompressible Navier-Stokes equations in curvilinear domains with complex immersed boundaries. Journal of Computational Physics, 225(2):1782-1809, 2007.

[27] I. Borazjani, L. Ge, and F. Sotiropoulos. Curvilinear immersed boundary method for simulating fluid - structure interaction with complex $3 \mathrm{~d}$ rigid bodies. Journal of Computational Physics, 227(16):7587-7620, 2008.

[28] Antoni Calderer, Seokkoo Kang, and Fotis Sotiropoulos. Level set immersed boundary method for coupled simulation of air/water interaction with complex floating structures. Journal of Computational Physics, 277:201-227, 2014.

[29] Seokkoo Kang, Anne Lightbody, Craig Hill, and Fotis Sotiropoulos. High-resolution numerical simulation of turbulence in natural water ways. Adv Water Resour, 34:98113, 2011.

[30] Seokkoo Kang, Xiaolei Yang, and Fotis Sotiropoulos. On the onset of wake mean- 
dering for an axial flow turbine in a turbulent open channel flow. J. Fluid Mech., 744:376-403, 2014.

[31] Ali Khosronejad and Fotis Sotiropoulos. Numerical simulation of sand waves in a turbulent open channel flow. Journal of Fluid Mechanics, 753:150-216, 2014.

[32] Daegyoum Kim, Julia Cossé, Cecilia Huertas Cerdeira, and Morteza Gharib. Flapping dynamics of an inverted flag. J. Fluid Mech., 736, 2013.

[33] S Kang, I Borazjani, J Colby, and F Sotiropoulos. Numerical simulation of 3d flow past a real - life marine hydrokinetic turbine. Adv Water Resour, 39:33-43, 2012.

[34] M. Germano, U. Piomelli, P. Moin, and W. H. Cabot. A dynamic subgrid-scale eddy viscosity model. Physics of Fluids, 3(7):1760-1765, 1991.

[35] Christopher W. Macosko. Rheology: Principles, Measurements, and Applications. Wiley VCH, 1994.

[36] S. Timoshenko and S. Woinowsky-Krieger. Theory of plates and shells. McGraw-Hill New York, 1959.

[37] I.M. Smith and D.V. Griffith. Programming the finite element method. Willey and Son Ltd, 2004.

[38] N. Newmark. A method of computation for structural dynamics. Journal of Engineering Mechanics Devision, 85:67-94, 1959.

[39] A. Gilmanov, F. Sotiropoulos, and E. Balaras. A general reconstruction algorithm for simulating flows with complex 3D immersed boundaries on Cartesian grids. $J$. Comput. Phys., 191:660-669, 2003.

[40] Carlos A. Felippa, K. C. Park, and Charbel Farhat. Partitioned analysis of coupled mechanical systems. Computer Methods in Applied Mechanics and Engineering, 190(24-25):3247 - 3270, 2001.

[41] MA Fernandez, J-F Gerbeau, and C. Grandmont. A projection semi-implicit scheme for the coupling of an elastic structure with an incompressible fluid. International Journal for Numerical Methods in Engineering, 69:794-821, 2007.

[42] Ulrich Küttler and Wolfgang Wall. Fixed - point fluid - structure interaction solvers with dynamic relaxation. Computational Mechanics, 43:61-72, 2008.

[43] Wolfgang A. Wall and Ekkehard Ramm. Fluid-structure interaction based upon a stabilized (ALE) finite element method. COMPUTATIONAL MECHANICS. New Trends and Applications S.R. Idelsohn, E. Oate and E.N. Dvorkin (Eds.)CIMNE, Barcelona, Spain 1998, pages 1-20, 1998.

[44] Bjorn Hübner, Elmar Walhorn, and Dieter Dinkler. A monolithic approach to fluid structure interaction using spacetime finite elements. Computers $\&$ Fluids, 193:20872104, 2004.

[45] J.G. Valdes. Nonlinear analysis of orthotropic membrane and shell structures inncluding fluid - structure interaction. PHD Thesis. Polytechnic University of Catalonia. Department of materials structures and engineering. Polytechnic University of Catalonia, 2007.

[46] Hermann G. Matthies and Jan Steindorf. Partitioned strong coupling algorithms for fluid - structure interaction. Computers \&5 Structures, 81:805 - 812, 2003.

[47] C. Wood, A. J. Gil, O. Hassan, and J. Bonet. A partitioned coupling approach for dynamic fluid - structure interaction with applications to biological membranes. Int. J. Numer. Meth. Fluids, 57:555-581, 2008. 
[48] J.H. Argyris. Dynamics of structures. Elsevier Science Publishers B.V., 1991.

[49] M. Wang and P. Moin. Dynamic wall modeling for large-eddy simulation of complex turbulent flows. Physics of Fluids, 14:2043-2051, 2002.

[50] J.C.R. Hunt, A.A. Wray, and P. Moin. Eddies, streams, and convergence zones in turbulent flows. Proc. 1988 Summer Program, Stanford N.A.S.A. Centre for Turb. Res., CTR-S88, 736:193-208, 1988.

[51] C.J. Carmody, G. Burriesci, I.C. Howard, and E.A. Patterson. An approach to the simulation of fluid - structure interaction in the aortic valve. Journal of Biomechanics, 39:158-169, 2006.

[52] Trung Bao Le and Fotis Sotiropoulos. Fluid-structure interaction of an aortic heart valve prosthesis driven by an animated anatomic left ventricle. Journal of Computational Physics, 244:41-62, 2013.

[53] J. S. Liu, P. C. Lu, and S. H. Chu. Turbulence characteristics downstream of bileaflet aortic valve prostheses. J. Biomech. Eng., 122:118-124, 2000.

[54] Trung Bao Le and Fotis Sotiropoulos. On the three-dimensional vortical structure of early diastolic flow in a patient-specific left ventricle. European Journal of Mechanics B/Fluids, 35, 2012.

[55] T.H. New and D. Tsovolos. Vortex behaviour and velocity characteristics of jets issuing from hybrid inclined elliptic nozzles. Flow, Turbulence and Combustion, 89(4):601-625, 2012.

[56] M. S. Sacks, W.D. Merryman, and D.E. Schmidt. Heart valve function: a biomechanical perspective. Journal of Biomechanics, 42:1804-1824, 2009.

[57] Philippe Pibarot and Jean G. Dumesnil. Prosthetic heart valves. Selection of the optimal prosthesis and long-term management. Circulation, 119:1034 - 1048, 2009. 


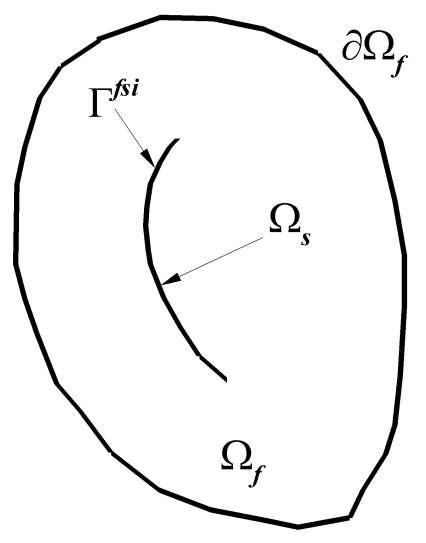

Fig. 1. Schematic of the computational domains for a FSI problem with a thin structure $\Omega_{s}$ immersed in a background fluid domain $\Omega_{f}$. The solid body is considered as a thin shell and $\Gamma^{f s i}$ is the interface between the fluid and solid domain intersection.
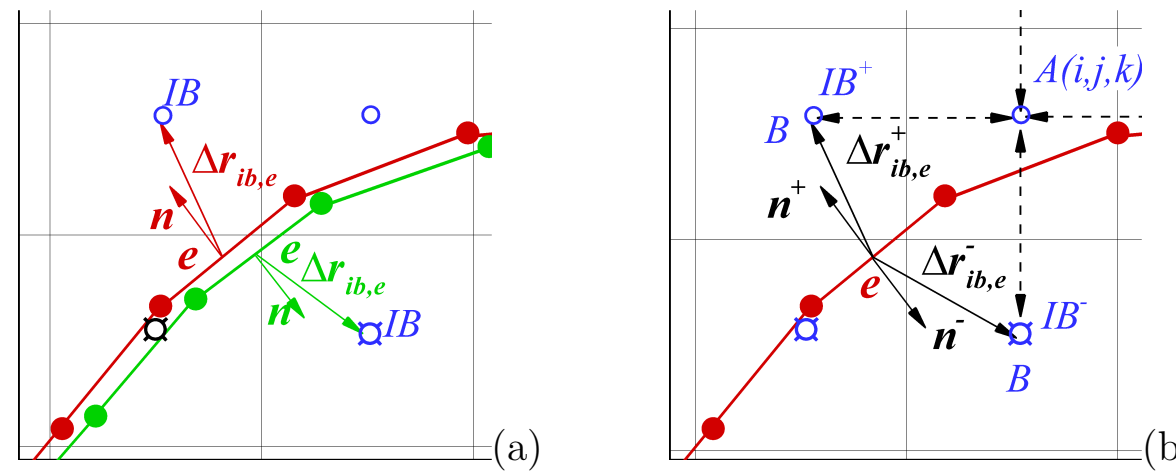

Fig. 2. Two different approaches for considering thin immersed body in the fluid. a) A closed body for which only the outward normal vector points toward the fluid side of the interface. The standard CURVIB formulation has been developed for such bodies. b) A surface with boundary (the mid surface of the thin structure). A two-side surface for which both the positive and negative surface normal vectors point toward the fluid side of the interface. In both sketches open circles denote background grid nodes (IB marks the immersed boundary nodes where boundary conditions are reconstructed), closed circles are Lagrangian points discretizing the body, and $e$ is the center of a structure shell element. Dashed lines indicate the direction of the searching algorithm from the background (fluid) grid node $A(i, j, k)$ to the adjacent points $B(i \pm 1, j \pm 1, k \pm 1)$. The points $I B^{+}$and $I B^{-}$in (b) indicate IB nodes from positive and negative side of the surface, respectively. 


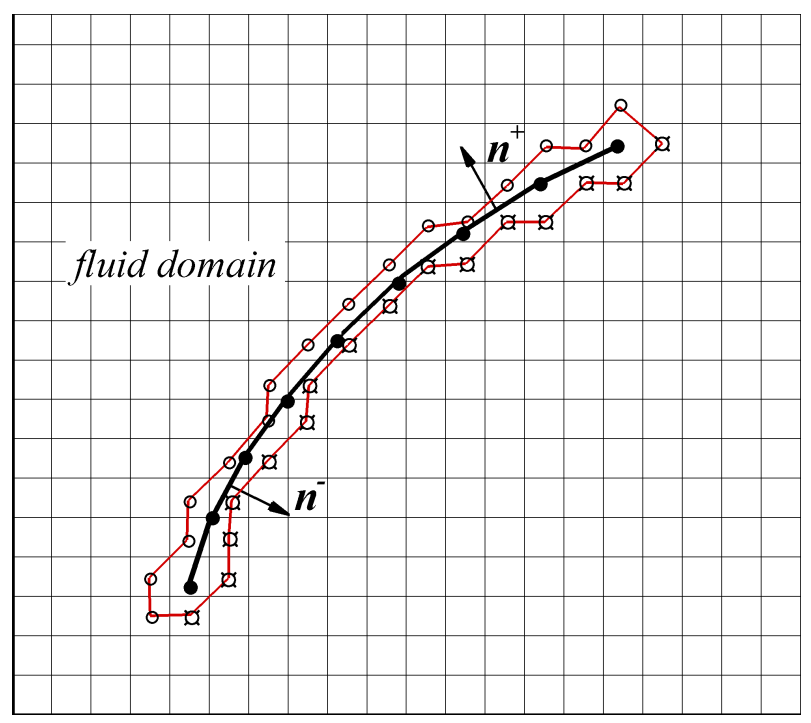

Fig. 3. The background grid where the governing equations for the fluid are solved along with the immersed thin body and associated IB nodes. Solid circles and solid line are vertices and elements of of the solid structure, respectively. Open circles are IB nodes from positive side and open circles with small primes are IB nodes from negative side of the surface. The red line marks the interface between the fluid domain and the layer of IB nodes surrounding the thin structure.

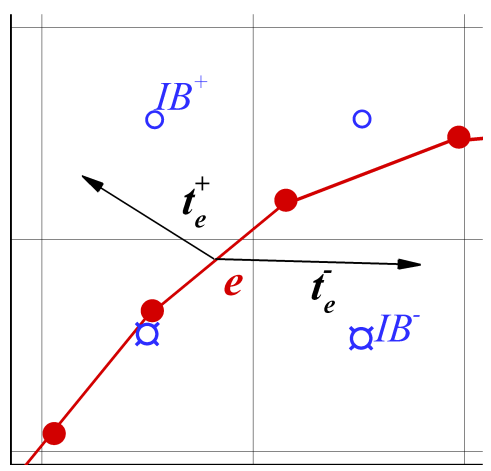

Fig. 4. The thin structure in our formulation is treated as a sharp interface. The schematic illustrates that at each element $e$ on the surface, two traction vectors are computed from the positive $\mathbf{t}_{e}^{+}$and negative $\mathbf{t}_{e}^{-}$sides of the interface. The total traction vector $\mathbf{t}_{e}=\mathbf{t}_{e}^{+}+\mathbf{t}_{e}^{-}$is used to compute the local load imparted by the flow on the structure on each surface element. 


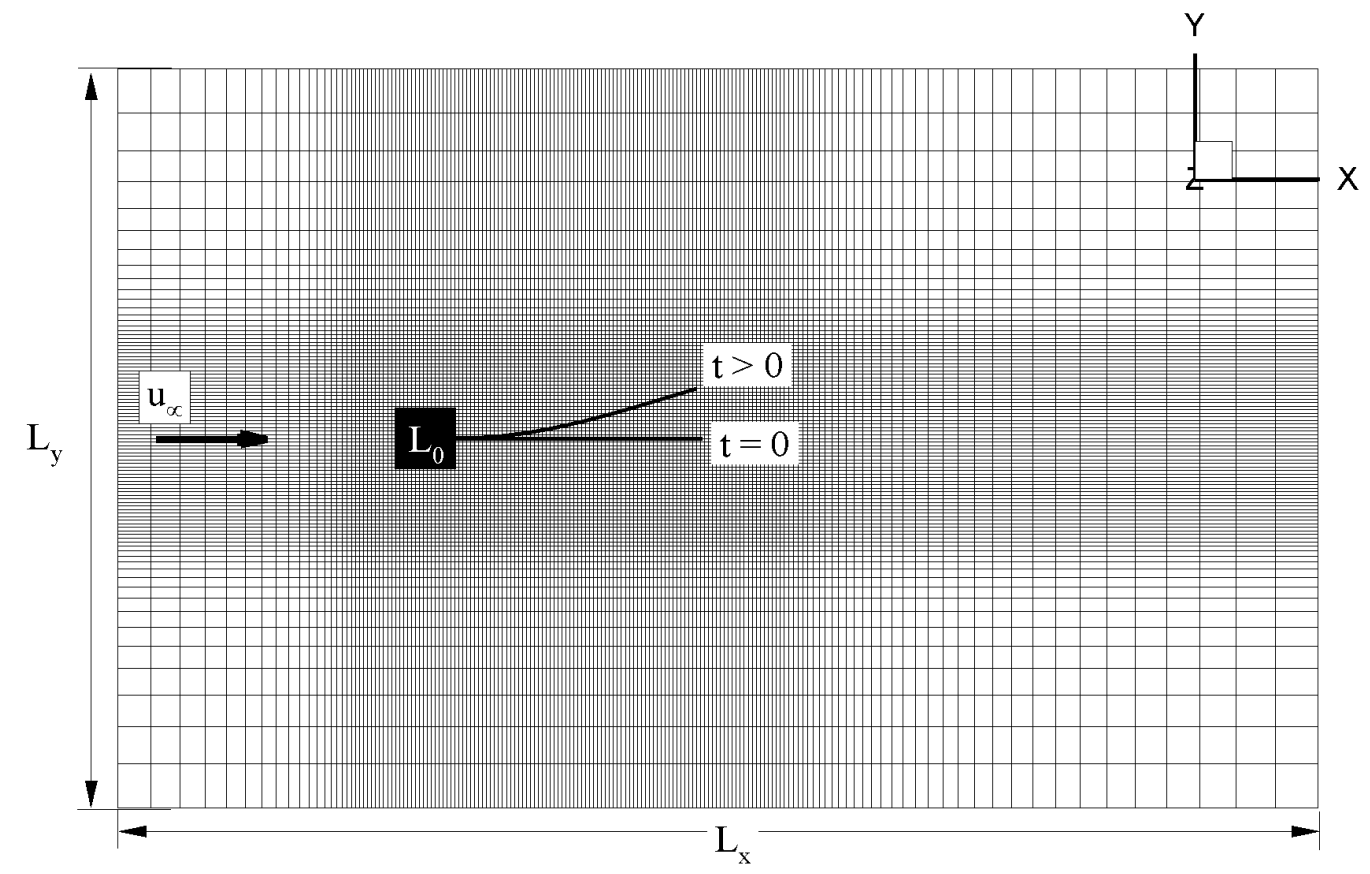

Fig. 5. Computational domain and representative background grid for a flexible cantilever mounted in the wake of a rigid square cylinder. $L_{x}=0.155 \mathrm{~m}$ and $L_{y}=0.12 \mathrm{~m}$ are the streamwise (x) and vertical (y) lengths of the computational domain, respectively. The left, bottom and top boundaries are Dirichlet boundaries with free stream $\left(u=u_{\infty}\right)$ and slip wall boundary conditions are specified. On the right boundary Neumann boundary condition is implemented: $\partial \mathbf{v} / \partial n=0$. We solve this $2 \mathrm{D}$ problem as $3 \mathrm{D}$ with symmetry boundary conditions in the transverse $(\mathrm{z})$ direction. 

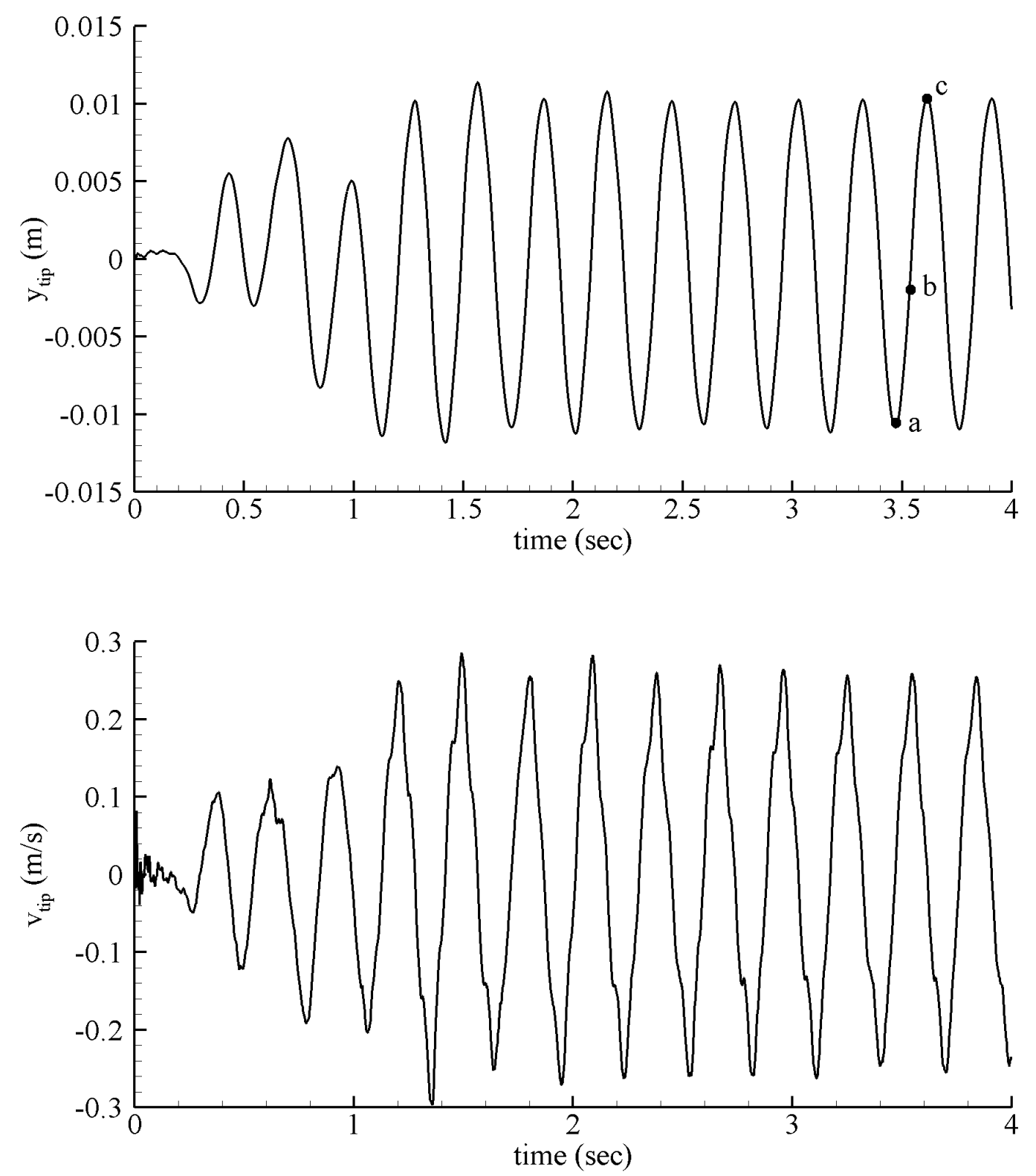

Fig. 6. Time history of the cantilever (a) tip displacement and (b) the tip velocity for the first mode of oscillations [43]. Points $a, b$, and $c$ indicate the time instants shown in Fig. 7. 

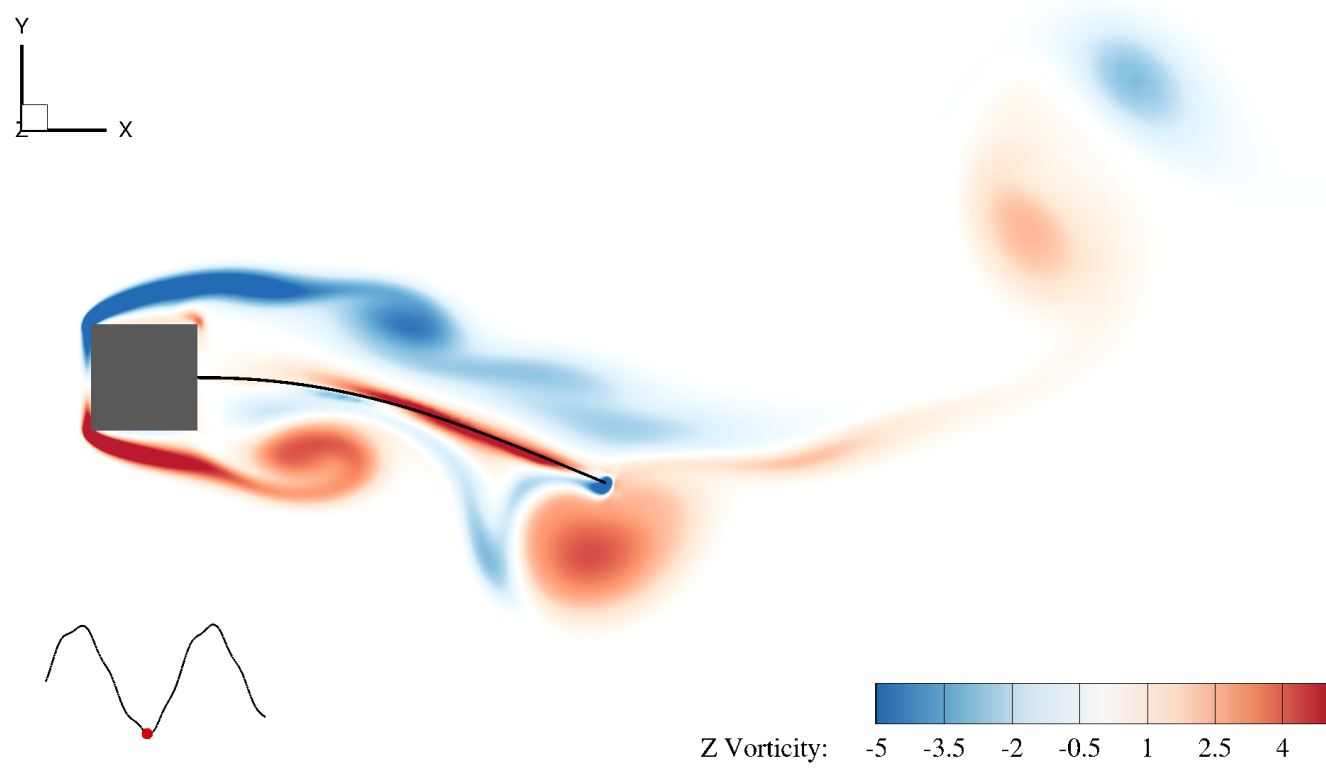

(a)
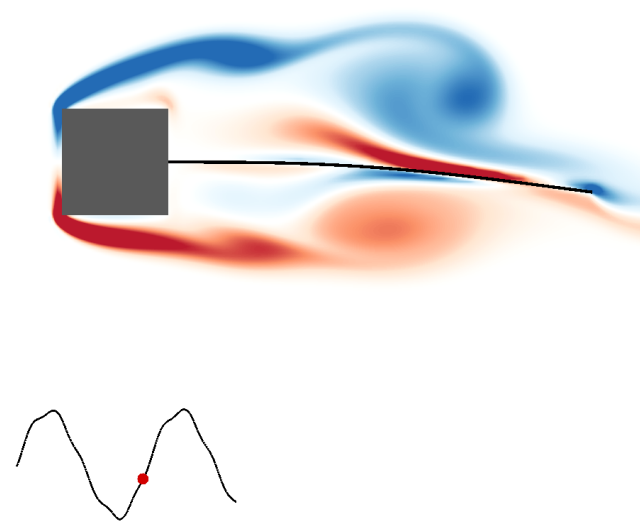

(b)
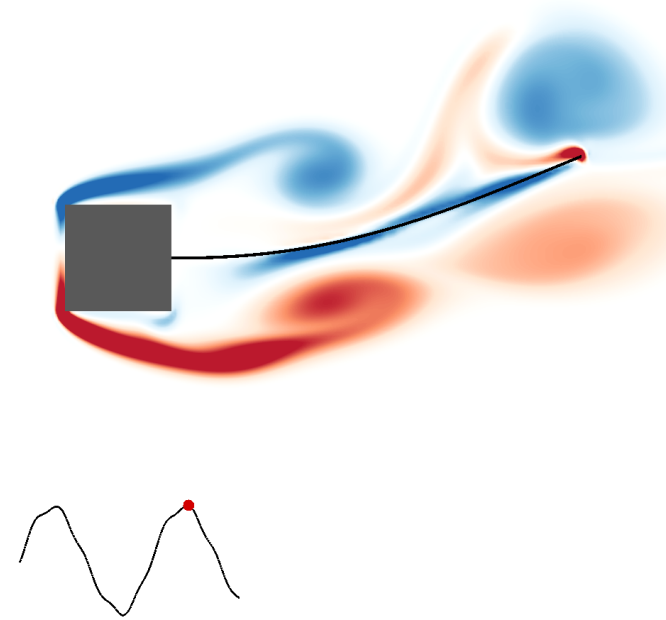

(c)

Fig. 7. Contours of instantaneous out of plane vorticity contours for the first mode of oscillation. The red dot in the inset of each figure identifies the instants corresponding Fig. 6: (a) $t_{a}=4.41 \mathrm{~s}$, (b) $t_{b}=4.46 \mathrm{~s}$, (c) $t_{c}=4.53 \mathrm{~s}$. 


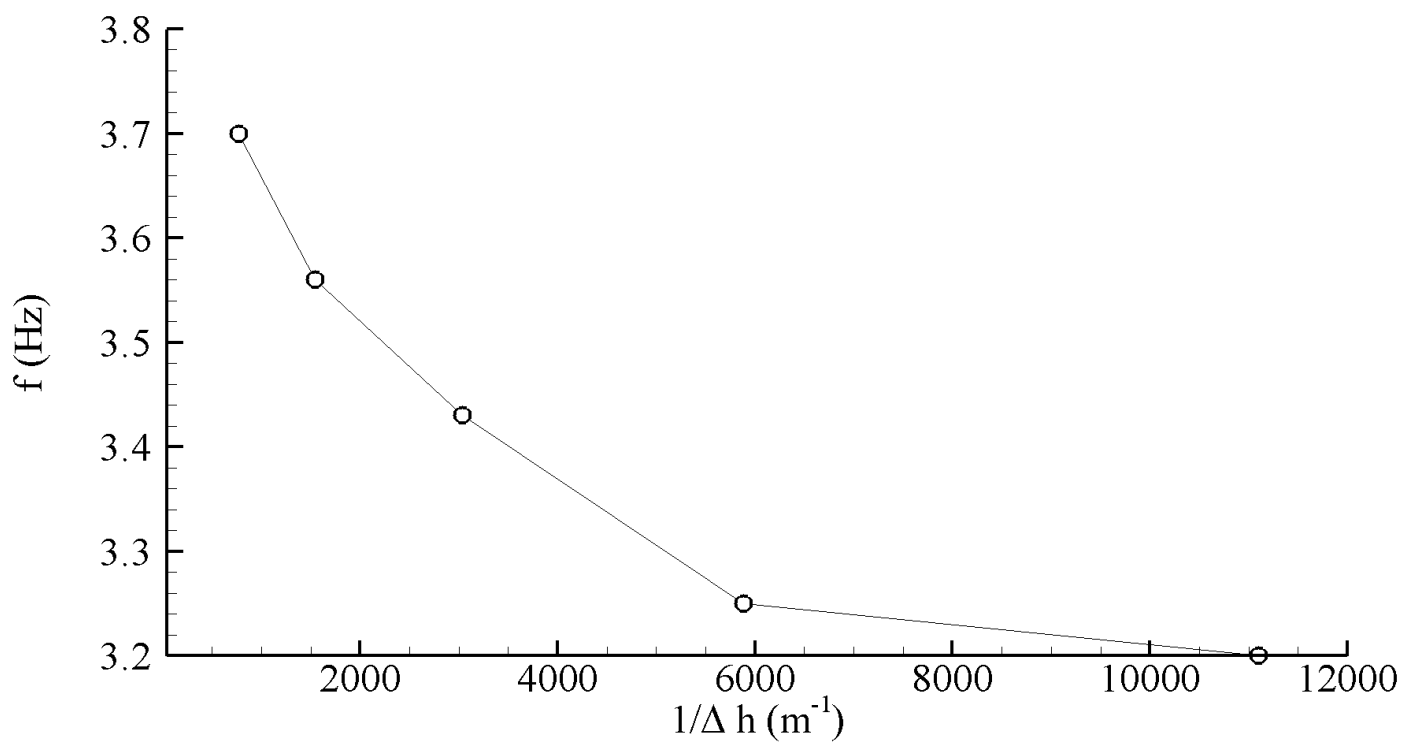

Fig. 8. Grid convergence study for the cantilever problem (1st mode of oscillations). The vibration frequency is plotted as function of the grid spacing $\Delta h$ in the vicinity of the structure for five successively finer grids: grid 1 $81 \times 45 \times 5$, grid $2-161 \times 91 \times 5$, grid $3-321 \times 181 \times 5$, grid $4-641 \times 365 \times 5$ and grid $5-1281 \times 721 \times 5$ grid nodes. 

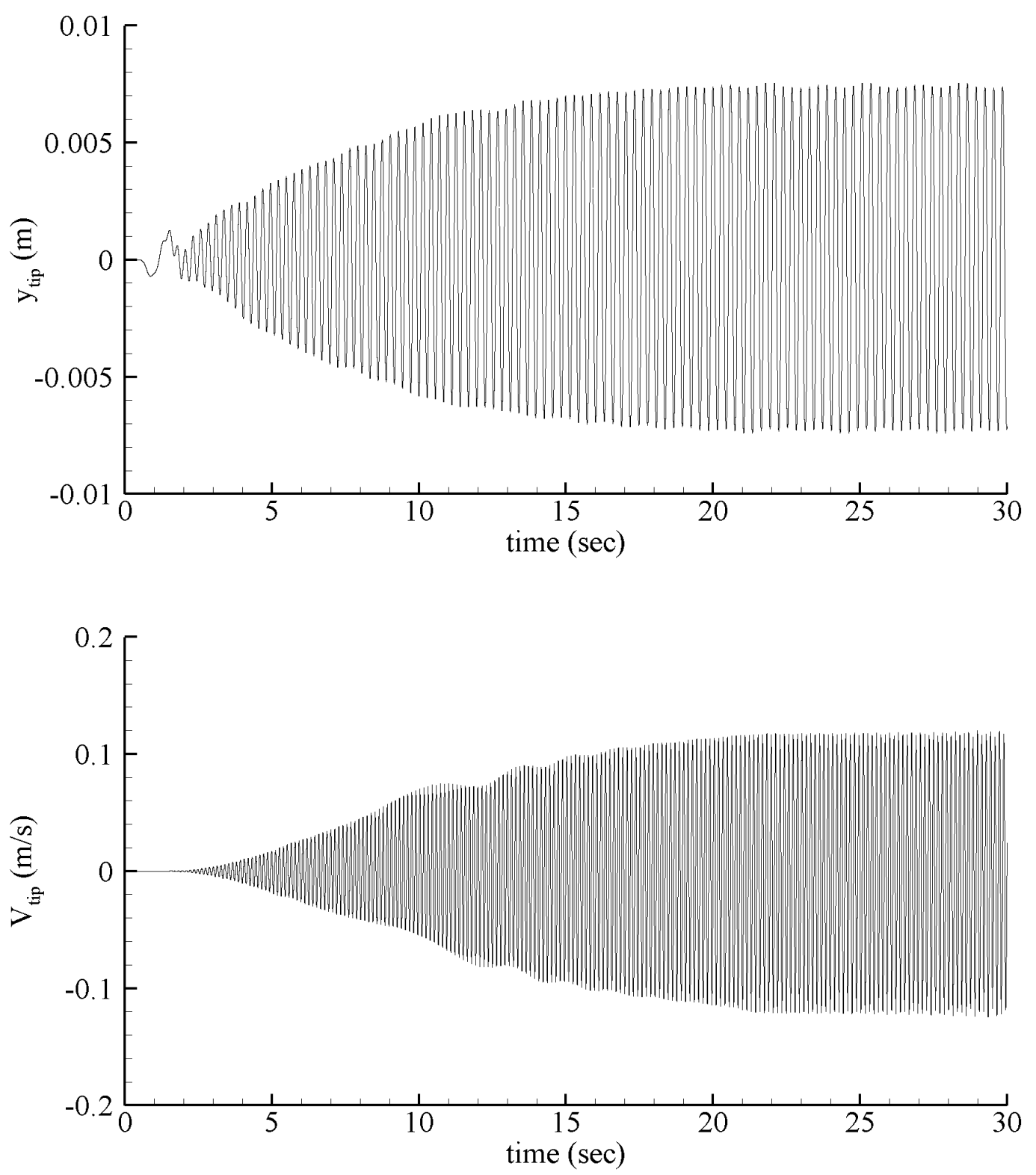

Fig. 9. Time history of the cantilever (a) tip displacement and (b) tip velocity for the second mode of oscillations [44]. 

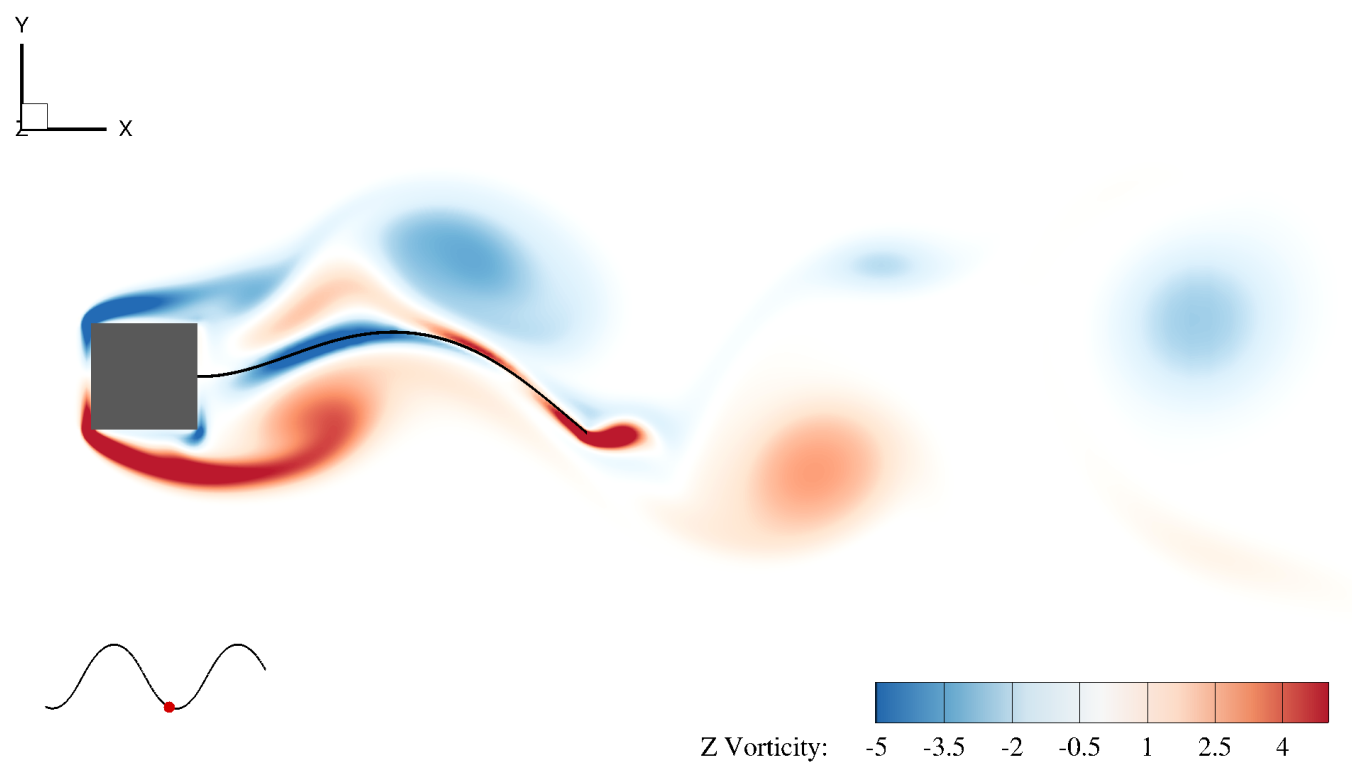

(a)

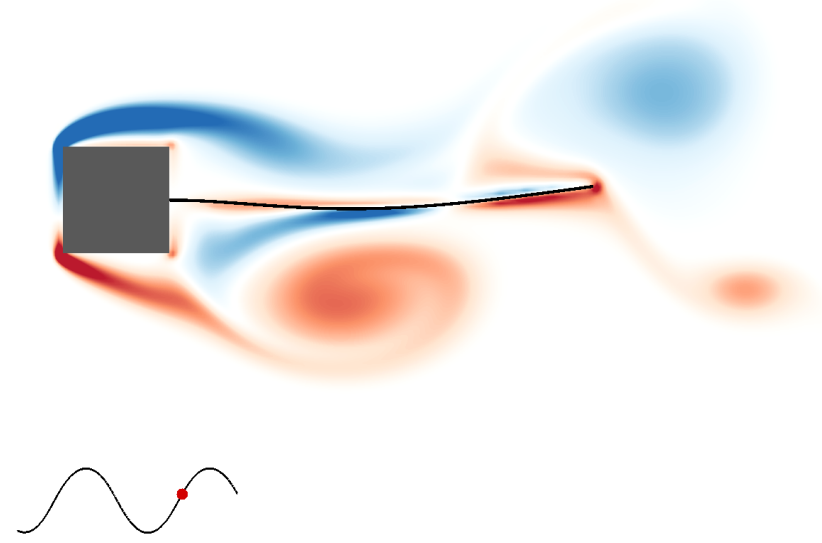

(b)

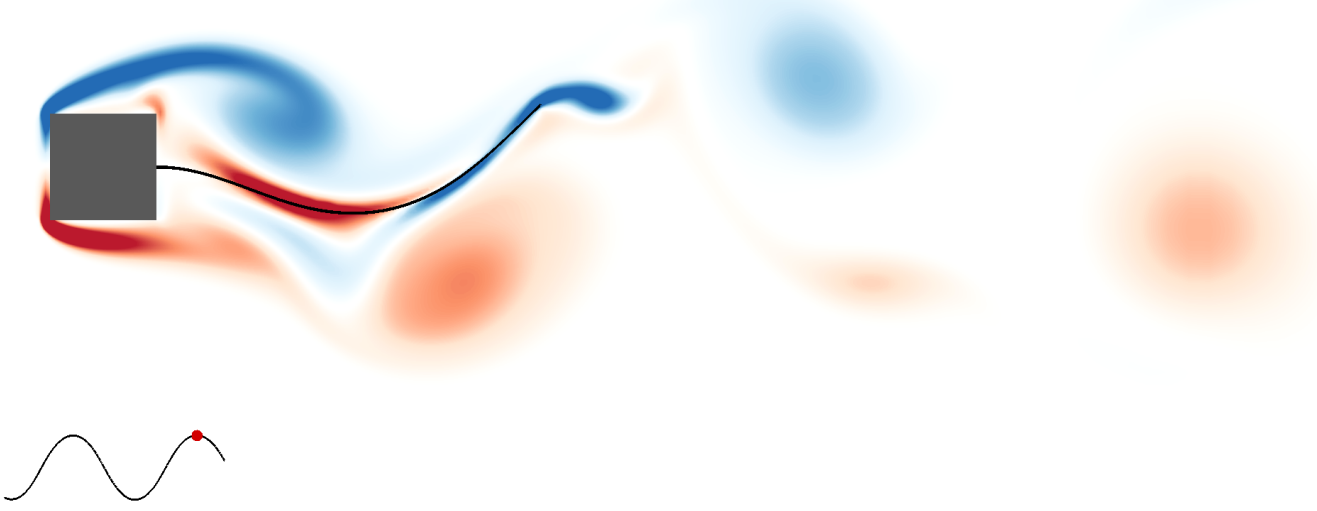

Fig. 10. Contours of instantaneous out of plane vorticity for the second mode of oscillation. The red dot in the inset of each figure identifies the corresponding instant during a half period of oscillations: (a) $t_{a}=15.6 \mathrm{~s}$, (b) $t_{b}=15.7 \mathrm{~s}$, (c) $t_{c}=15.8 \mathrm{~s}$ 


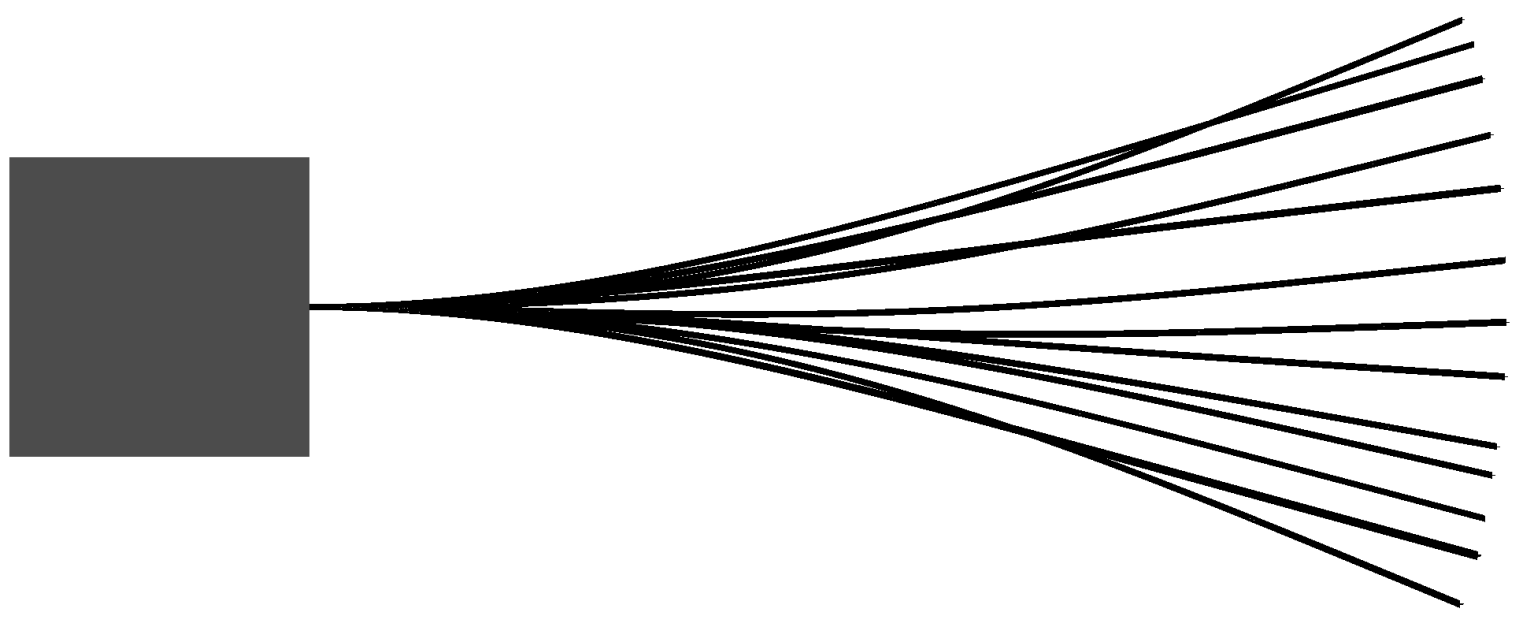

(a)

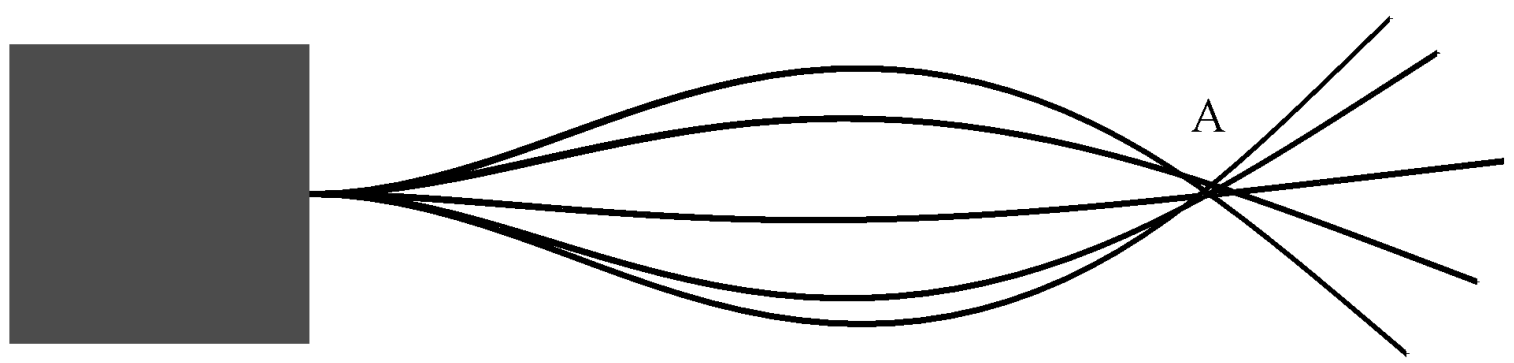

(b)

Fig. 11. Comparisons for the calculated two modes of cantilever oscillation: (a) 1st mode, and (b) 2nd mode with fixed point (A). Both figures show calculated instantaneous snapshots of the cantilever shapes. 


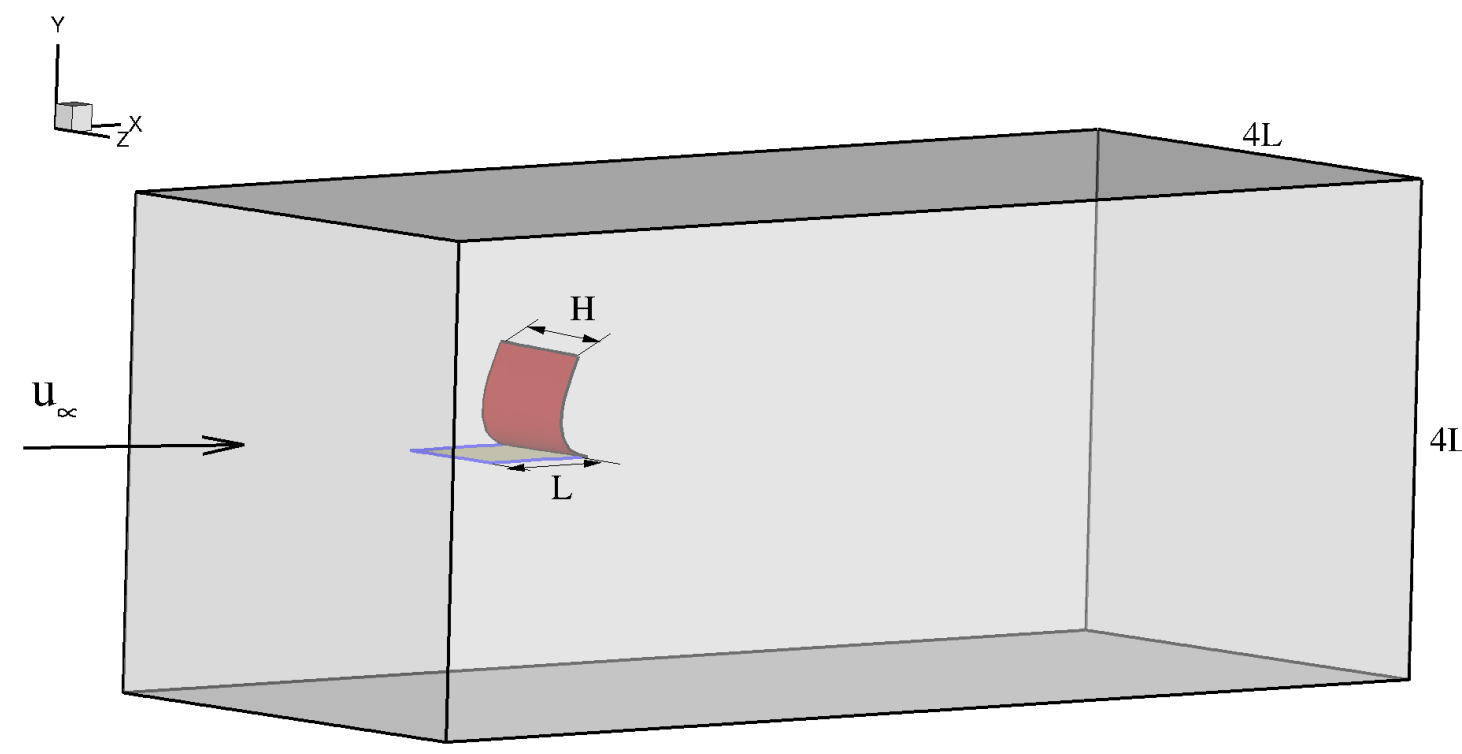

Fig. 12. Computational domain used to simulate the inverted flag problem. The side $x_{m i n}$ and all four sides $y_{\min }, y_{\max }, z_{\min }, z_{\max }$ are Dirichlet boundaries with inflow $u=u_{\infty}$ and no-slip boundary conditions $\mathbf{v}=0$, respectively. On the boundary $x_{\max }$ Neumann condition $\partial \mathbf{v} / \partial n=0$ is implemented.

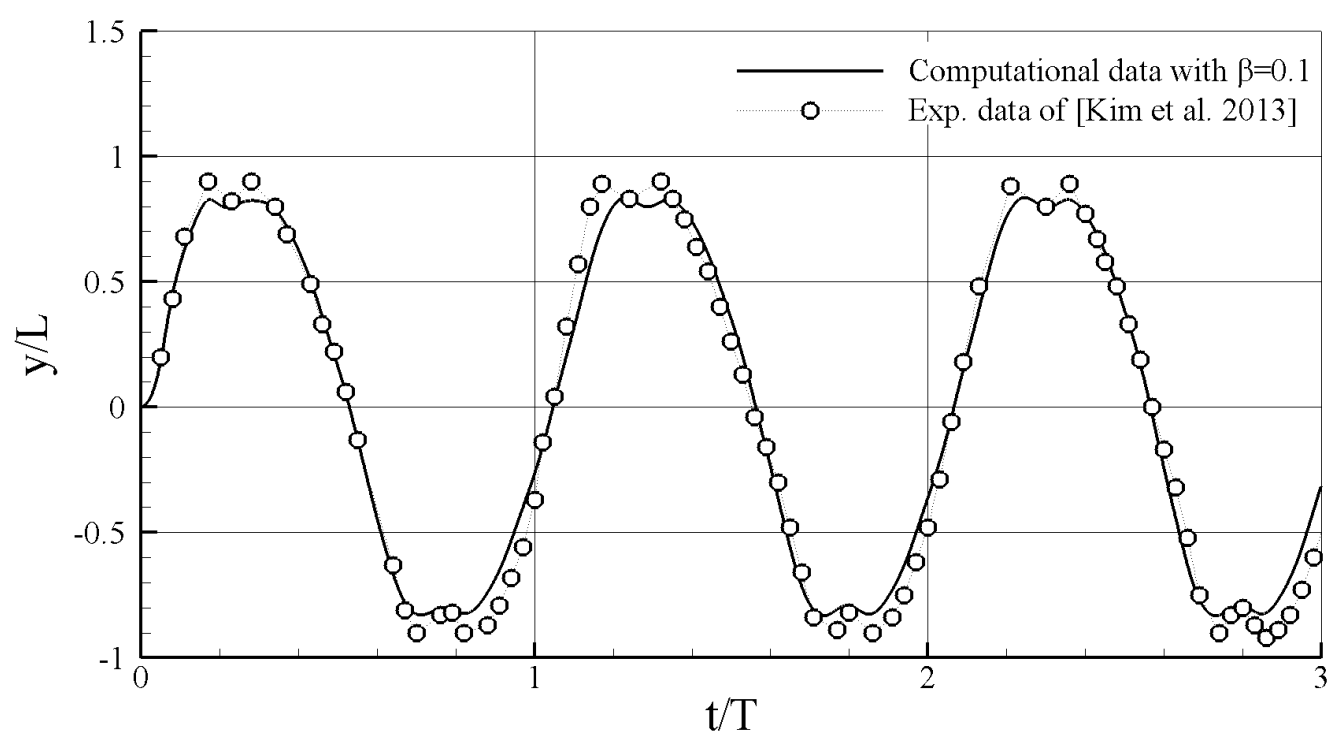

Fig. 13. Comparison of calculated (solid line) and measured [32] time histories of the flag leading edge displacements for flapping mode with $\beta=0.1$. Open circles are experimental data. 


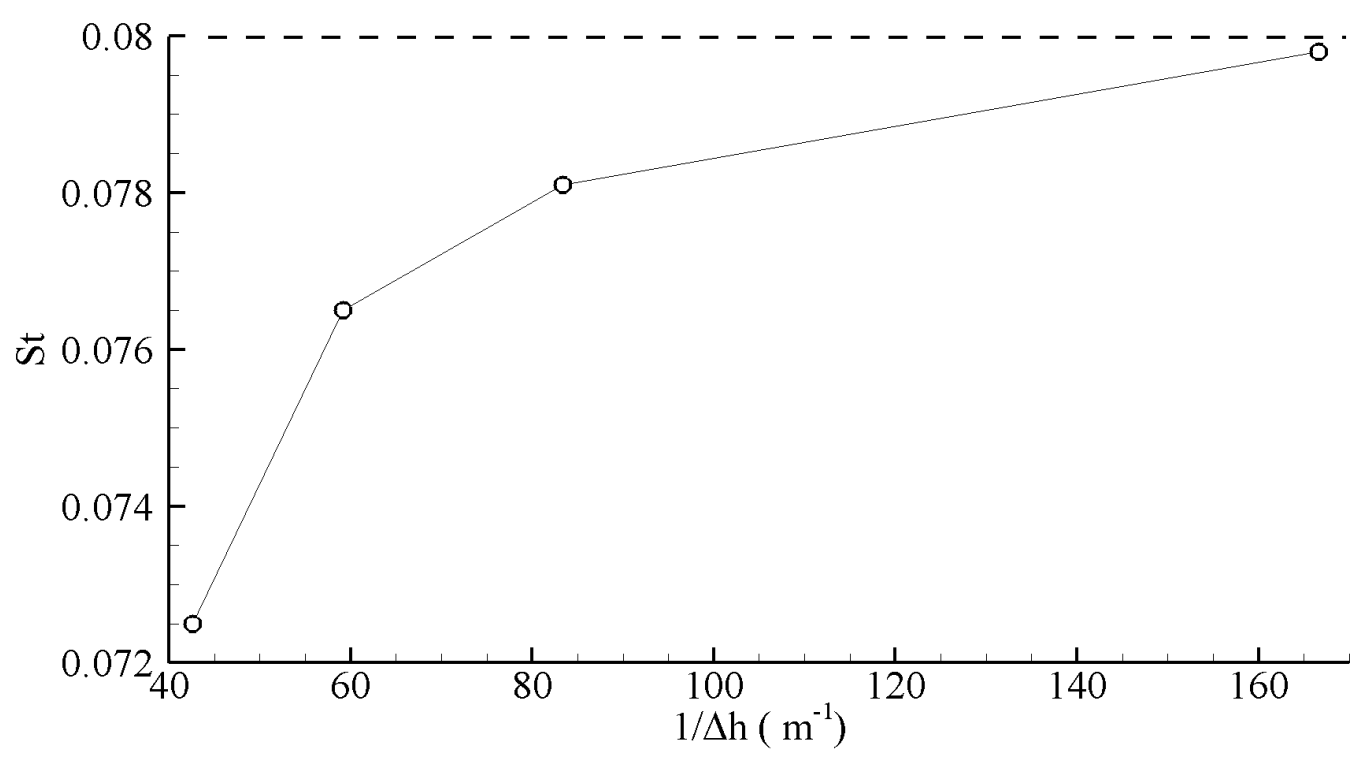

Fig. 14. Grid convergence study for the inverted flag problem. The flag Strouhal number (St) is plotted as function of the grid spacing $\Delta h$ on four successively finer grids: grid $1-141 \times 51 \times 51$; grid $2-201 \times 71 \times 71$; grid $3-281 \times 101 \times 101$ and gird $4-561 \times 201 \times 201$ grid nodes. Dashed line indicates the experimental value of St number (see Fig. $2 \mathrm{~b}$ in [32] for $\beta=0.1$ ).

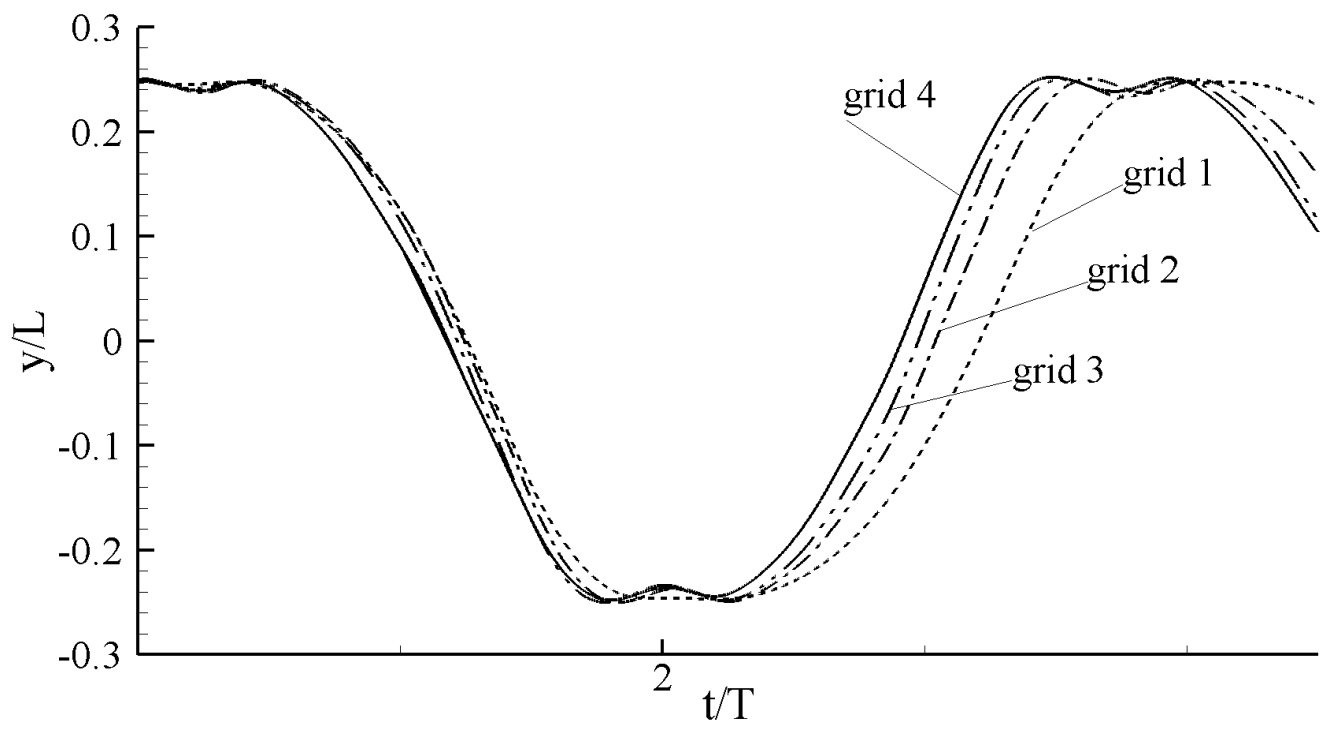

Fig. 15. Grid convergence study for the inverted flag problem. Time histories of the flag leading edge displacement for the flapping mode simulated on grid 1 (dashed line), grid 2 (dashed-dot line), grid 3 (dashed-dot-dot line) and grid 4 (solid line). See caption of Fig. 14 for definition of the various grid sizes. 


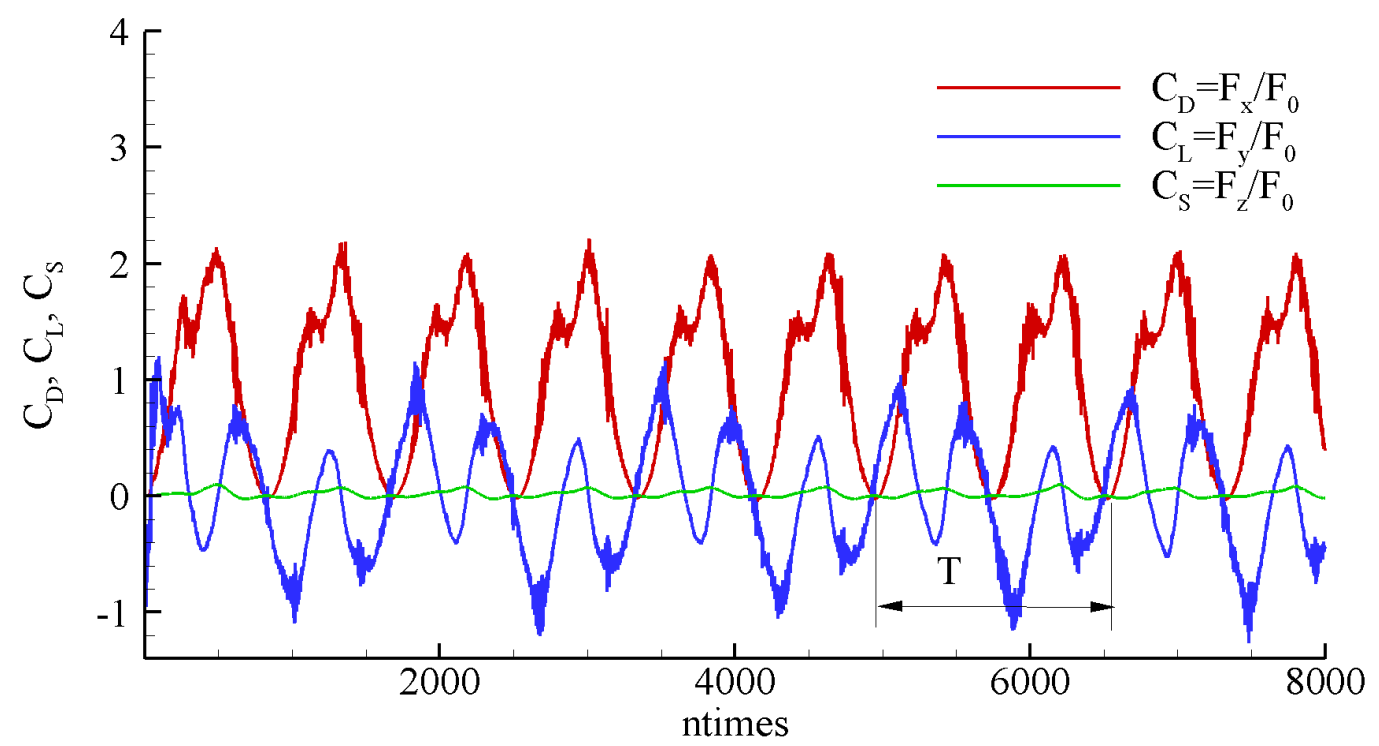

Fig. 16. Calculated time histories for the drag $\left(C_{D}\right)$, lift $\left(C_{L}\right)$, and span-wise $\left(C_{S}\right)$ coefficients normalized with $F_{0}=\rho_{f} u_{\infty}^{2} S / 2$, where $S=L H$ is a surface of the inverted flag, $F_{x}, F_{y}$ and $F_{z}$ are total forces in $\mathrm{x}, \mathrm{y}$ and $\mathrm{z}$ directions, respectively. Experimentally measured maximum and mean drag coefficients for $\beta=0.1$ are equal to $C_{D, \text { max }} \approx 2$ and $C_{D, \text { mean }} \approx 1$ respectively, (see [32], Fig.5a). Computed drag coefficients $C_{D, \text { max }}^{\text {compute }} \approx 2$ and $C_{D, \text { mean }}^{\text {comput }} \approx 1$ and are in excellent agreement with experimental values. Note that span-wise coefficient is not equal to 0, which implies twisting mode oscillations of the flag around its lengthwise axis as shown in Fig.17. 


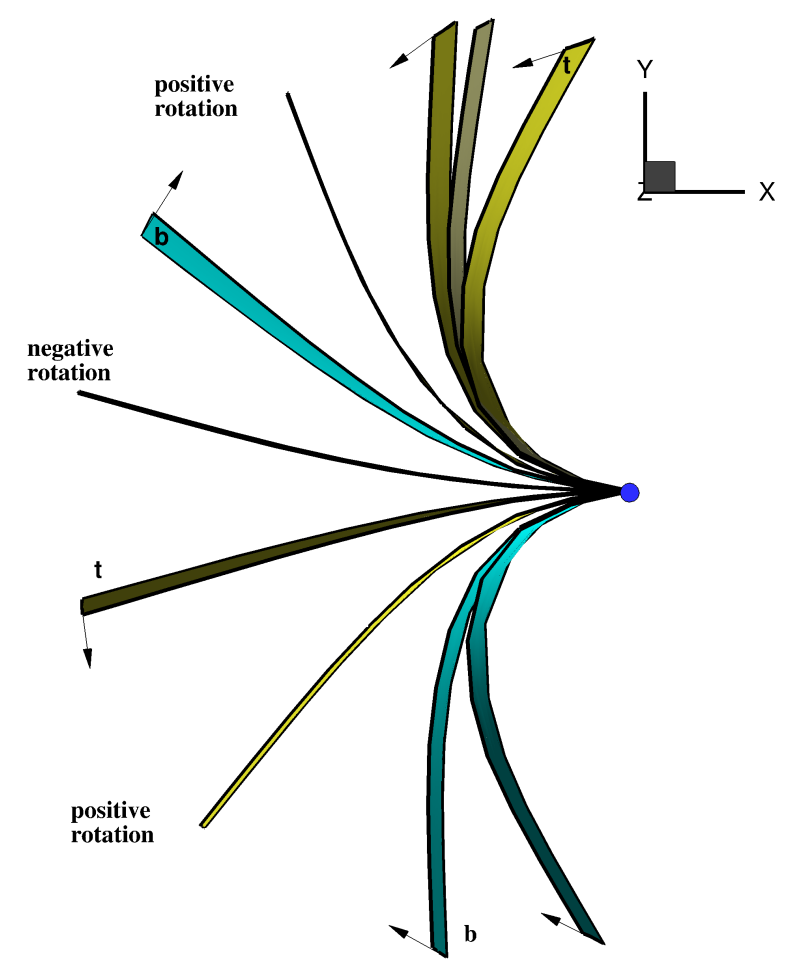

Fig. 17. Calculated instantaneous shapes of inverted flag during a half period of oscillations (from the highest to the lowest deflection) showing the onset of the twisting mode causing the flag to rotate around its longitudinal axis. Yellow and blue colors indicate top and bottom sides of the flag's surface. The blue point is the fixed trailing edge of the flag. Arrows are aligned with the leading edge of the flag to clearly show the positive (counterclockwise) and negative (clockwise) rotation of the flag around its longitudinal axis. 

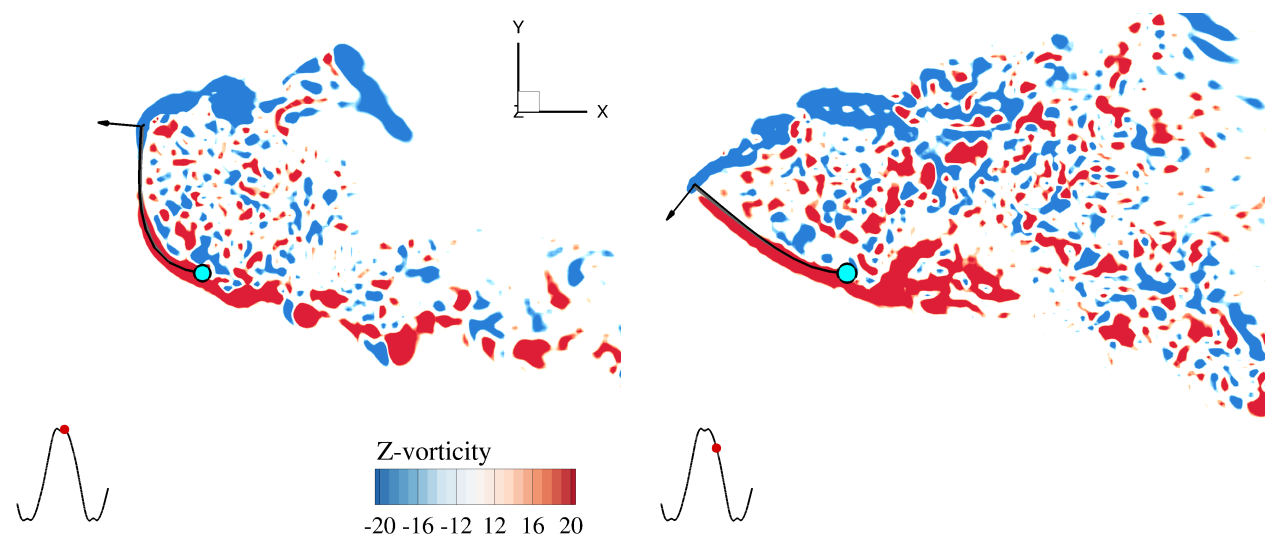

(a)

(b)

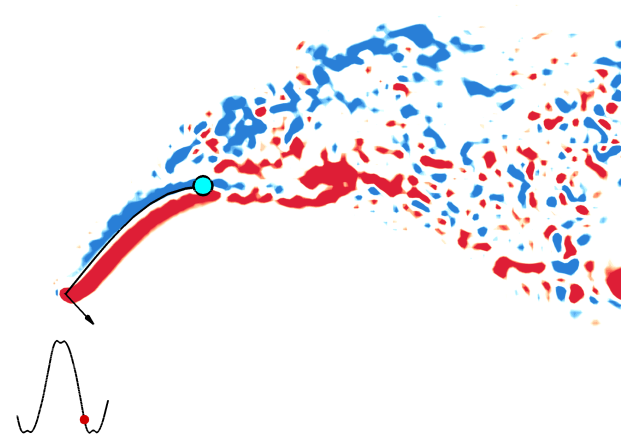

(c)

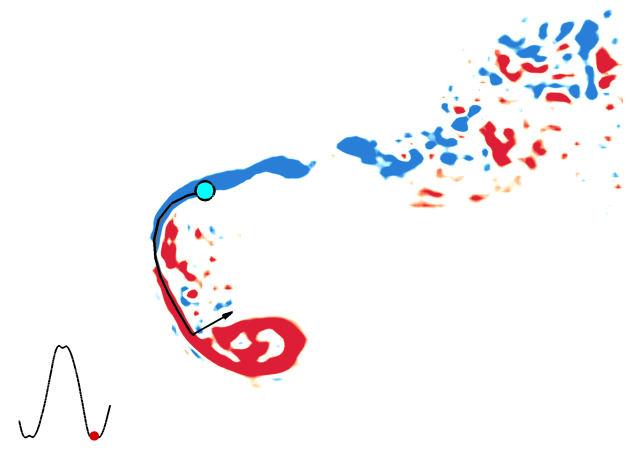

(d)

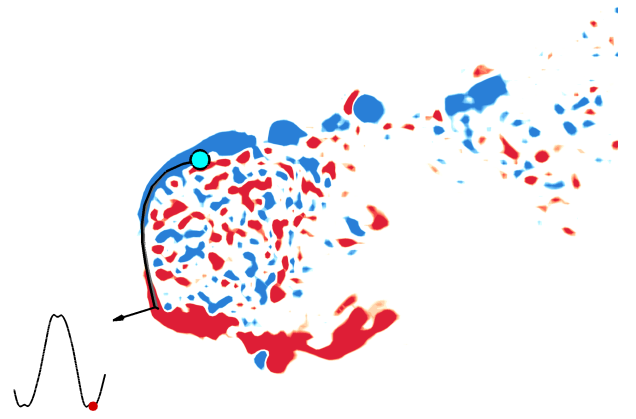

(e)

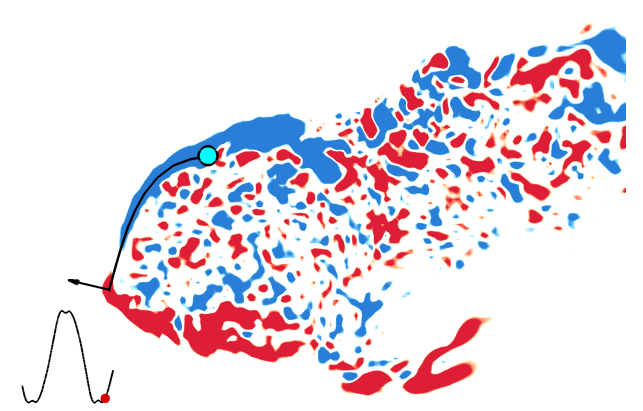

(f)

Fig. 18. Snapshots of the simulated inverted flag flowfields during a half period of oscillation. Contours are the out-of-plane vorticity component (z-vorticity) are plotted at various instants in time. The corresponding flag shape is also shown and the corresponding time instant is marked with a red dot in the inset. Light blue circle indicates the fixed trailing edge and the arrow indicates direction of moving leading edge. 


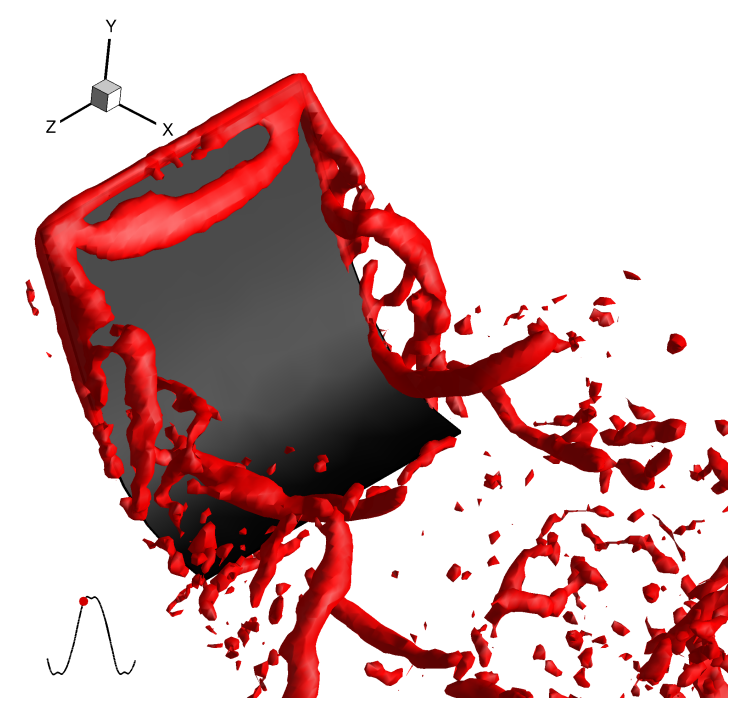

(a)

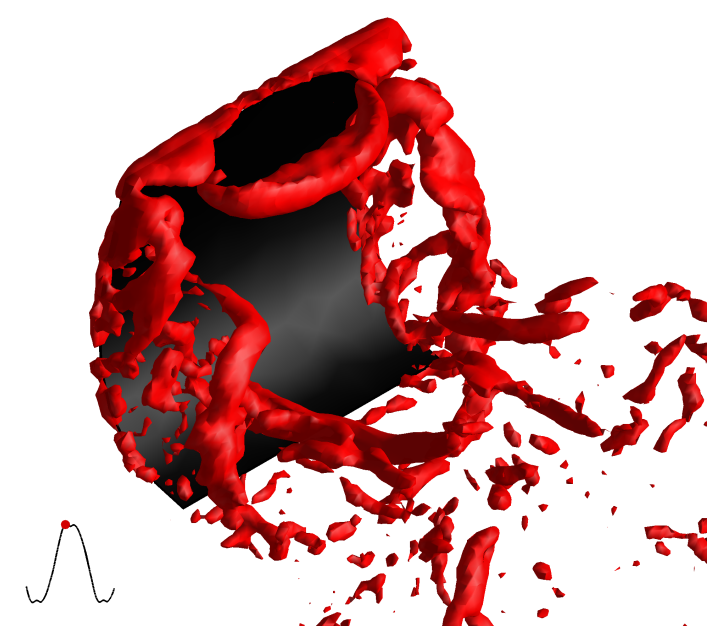

(b)

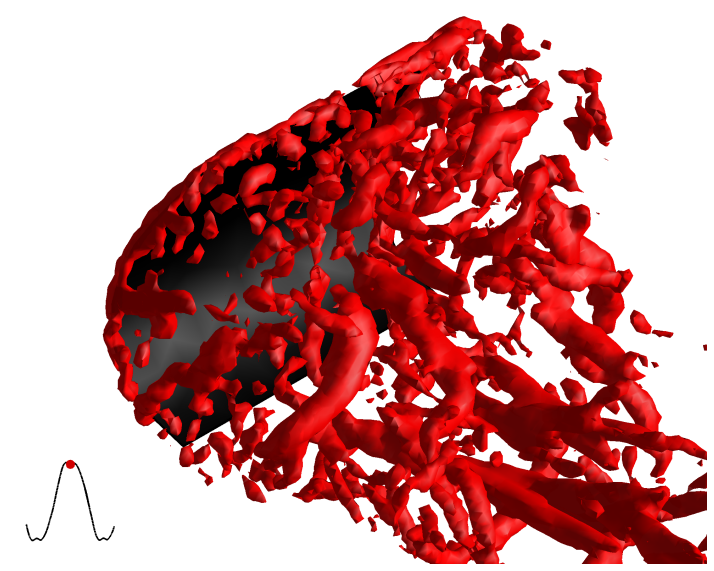

(c)

Fig. 19. Snapshots of iso-surfaces [50] at three tim 49 instants showing the inverted flag near the maximum deflection. These snapshots elucidate the 3D coherent structures in the wake of the flapping flag. The red dot in the inset of each figure identifies the corresponding instant. 


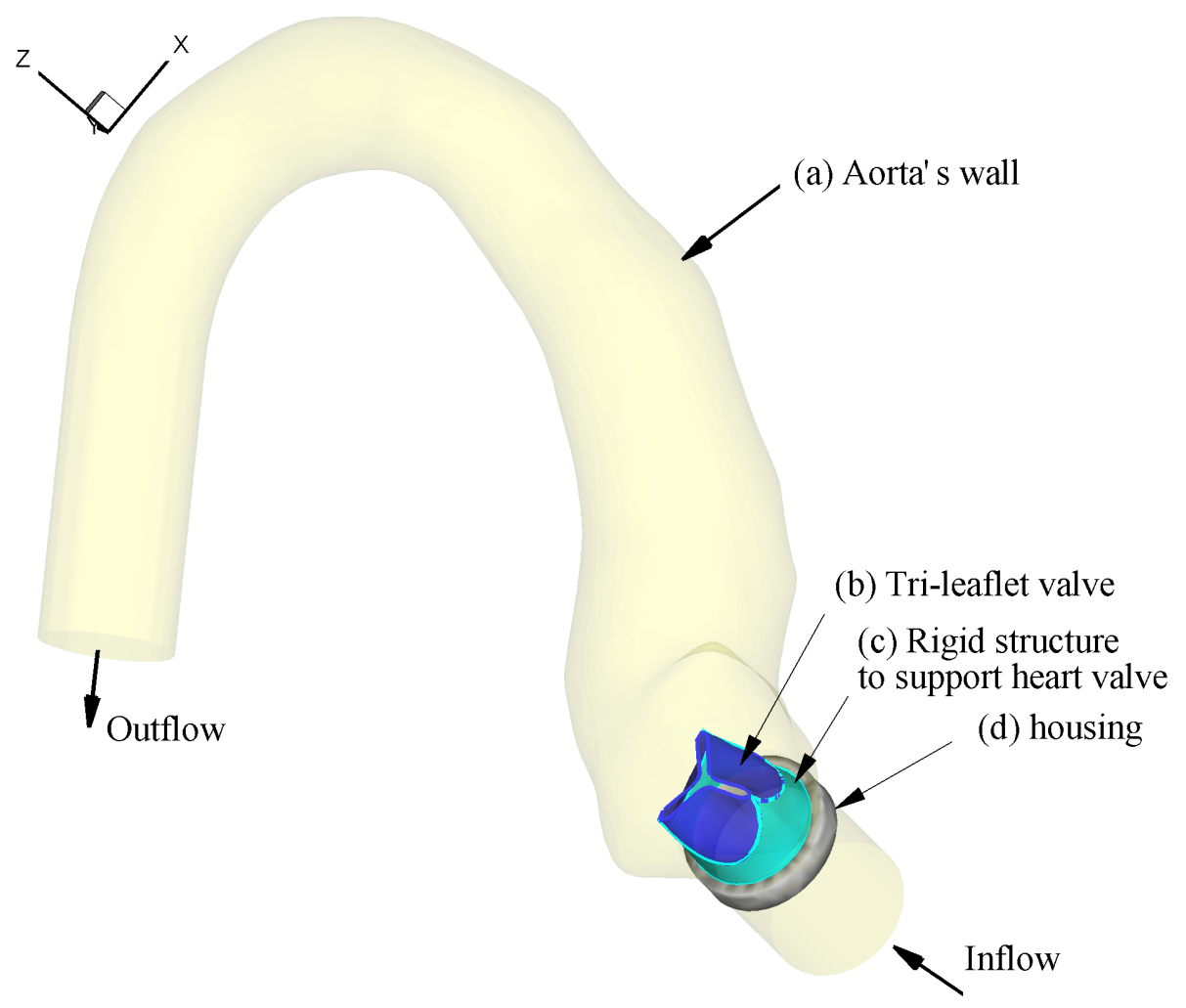

Fig. 20. Computational domain for the FSI simulations of a tri-leflet heart valve in an anatomic aorta. At inflow pulsatile physiological flow shown in Fig. 21 is simulated; at outflow Neumann boundary condition $\partial \mathbf{v} / \partial n=0$ is implemented; on the aorta wall no-slip boundary condition is implemented. 


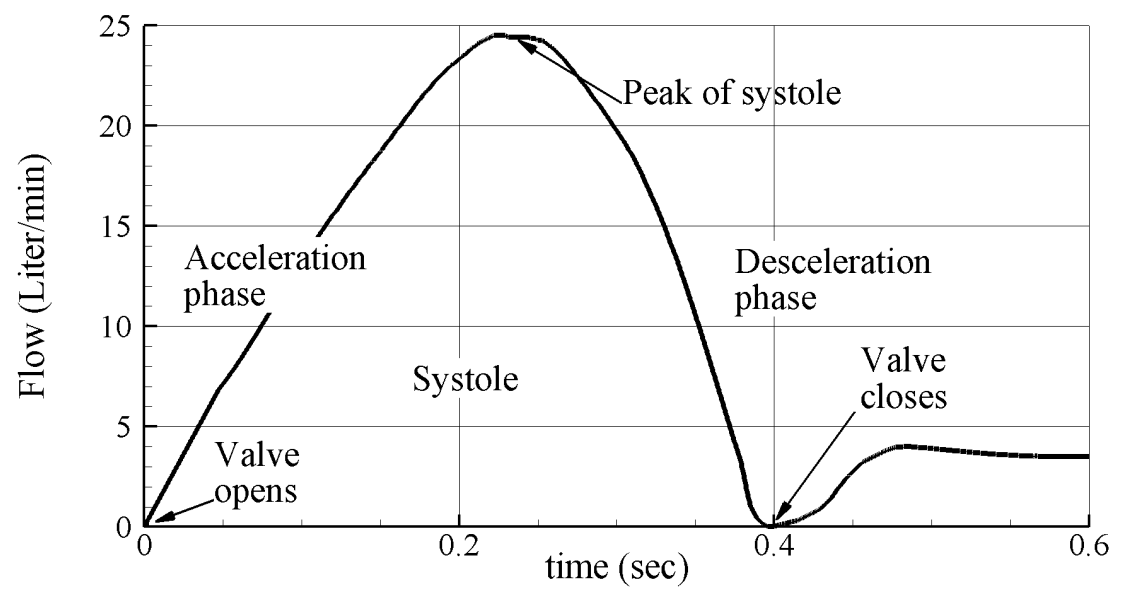

Fig. 21. Physiological incoming flow waveform specified at the inlet of the aorta. 


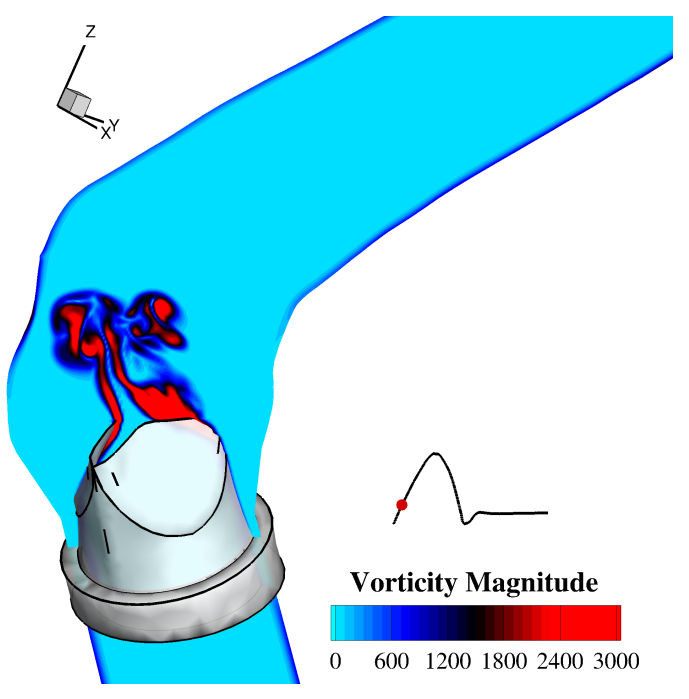

(a)

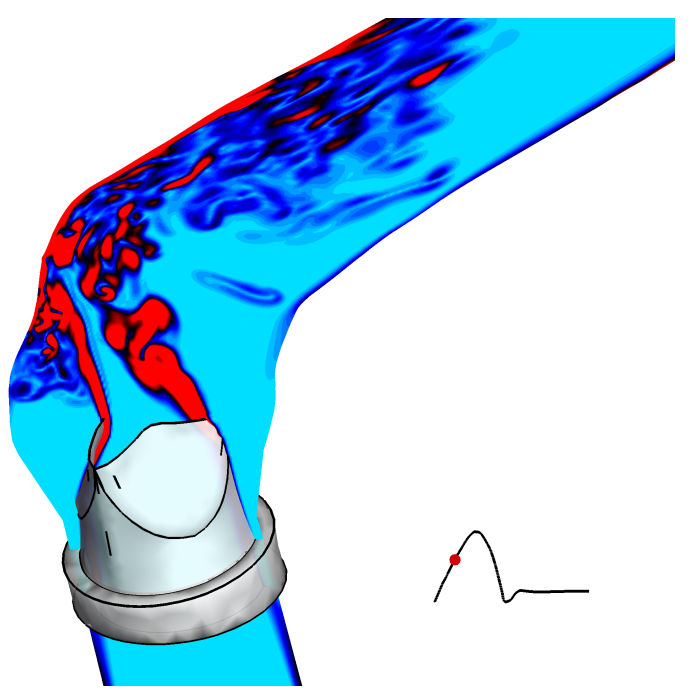

(c)

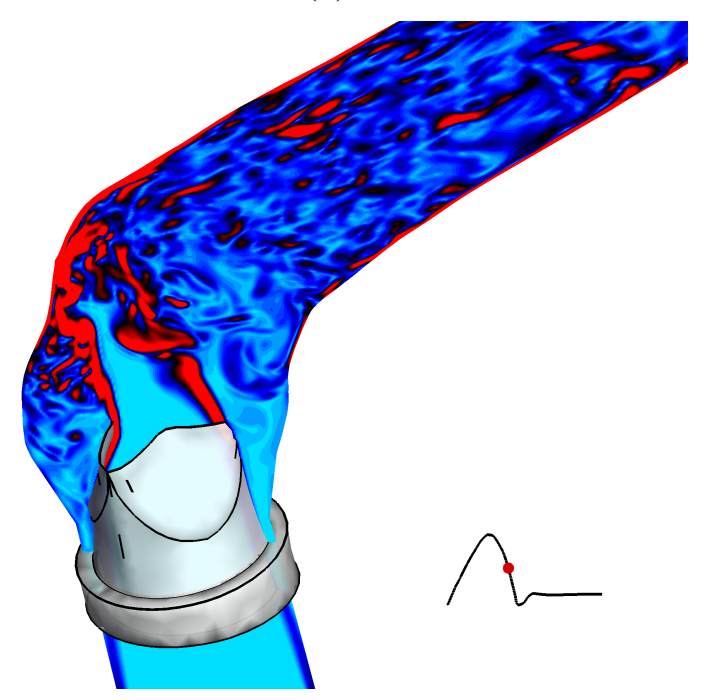

(e)

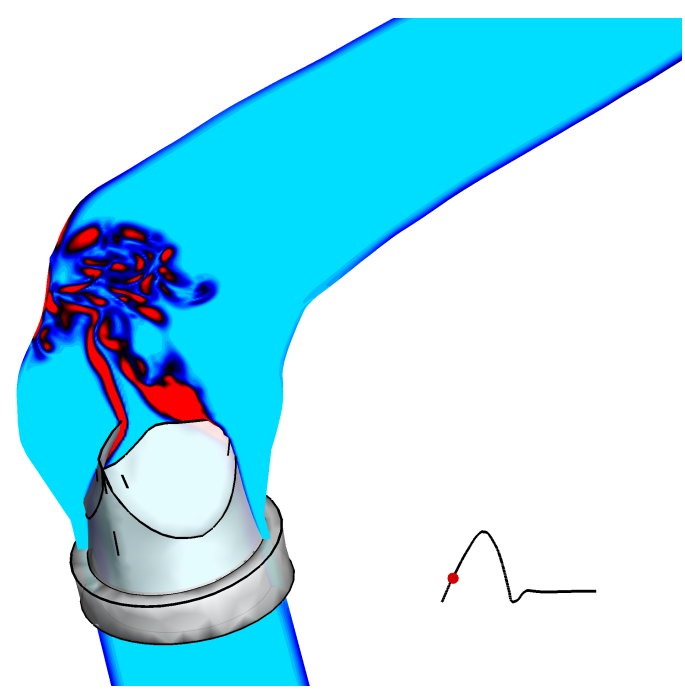

(b)

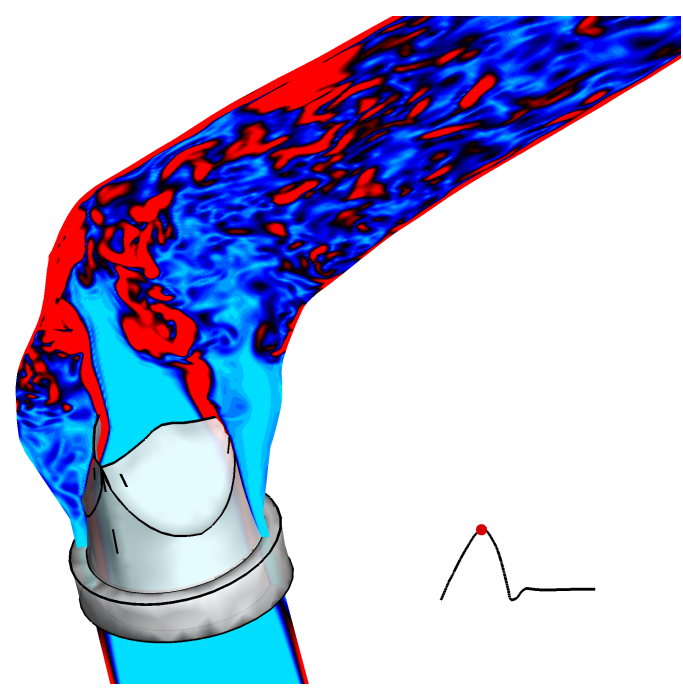

(d)

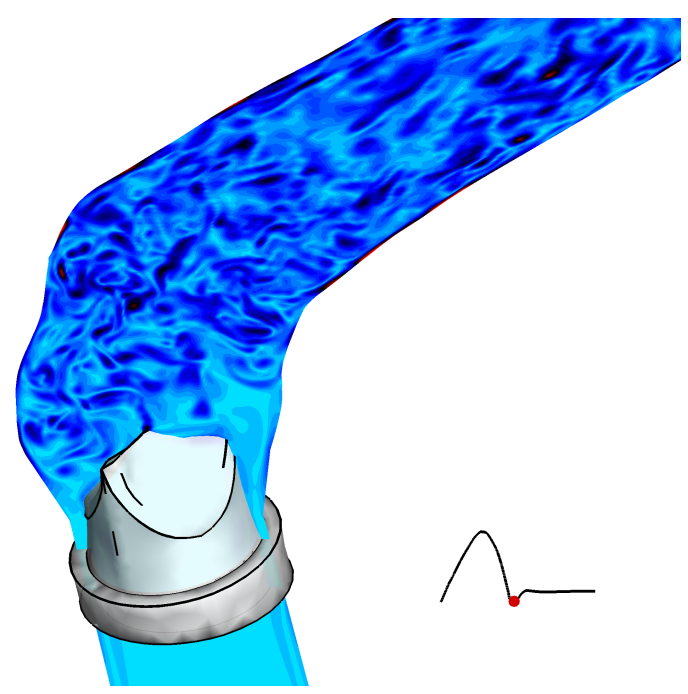

(f)

Fig. 22. Instantaneous contours of vorticity magnitude on a plane through the aorta plane of symmetry during diastolic phase showing the opening and closing process of the aortic heart valve. The red dot in the inset of each figure identifies the corresponding instant during the catdiac cycle. 


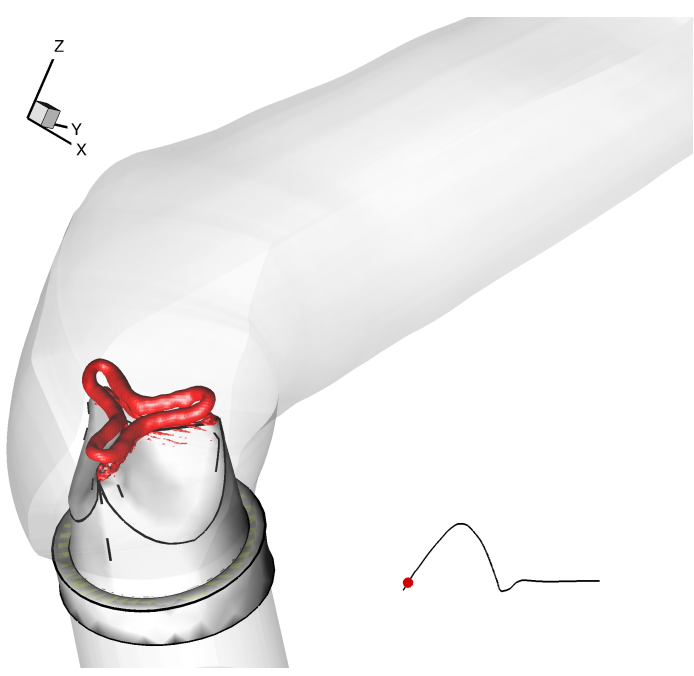

(a)

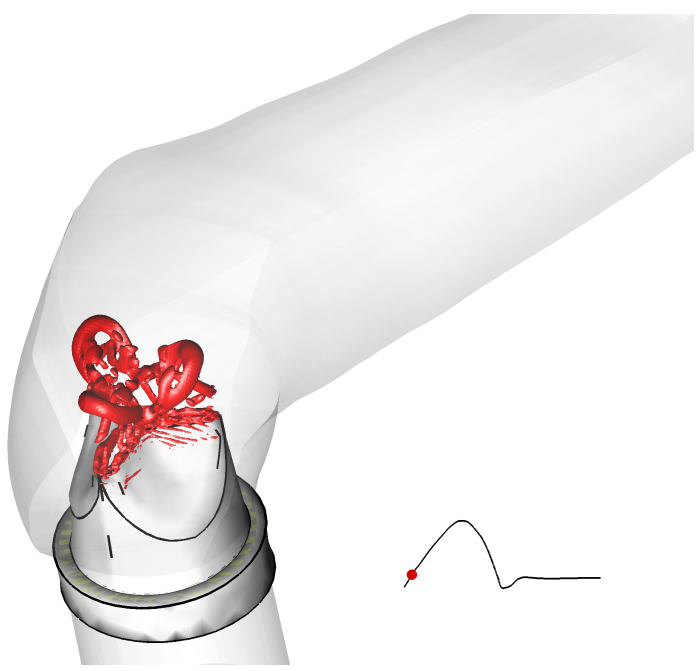

(c)

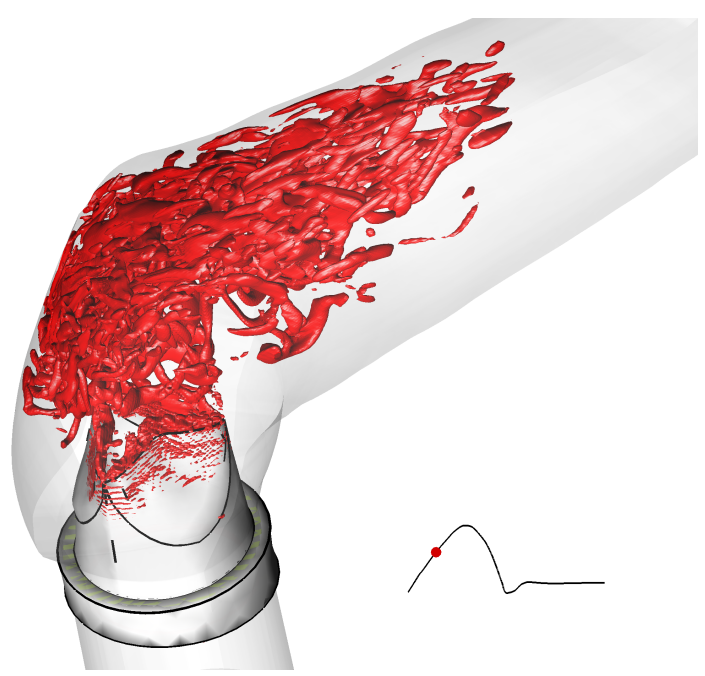

(e)

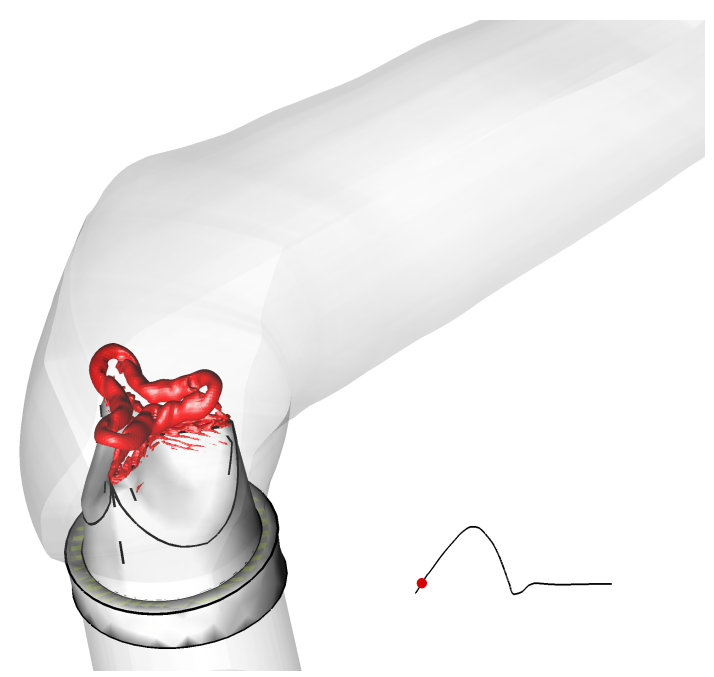

(b)

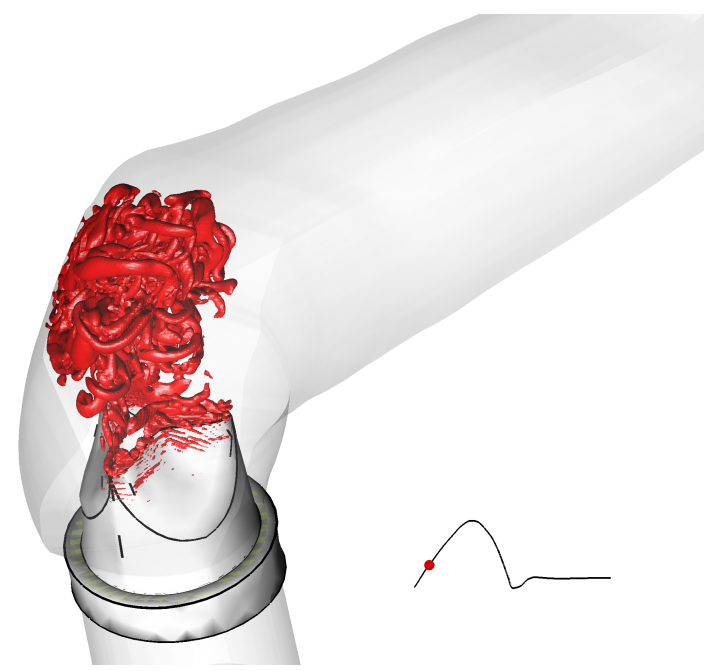

(d)

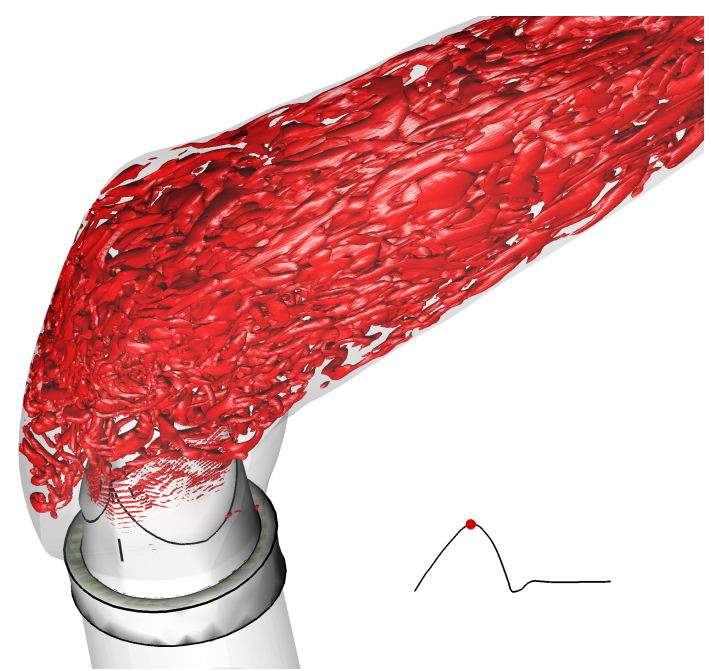

(f)

Fig. 23. Instantaneous iso-surfaces of the Q-criterion [50] at various instant in time during valve opening. The red dot in the inset of each figure identifies the corresponding instant during the cardiac cycle. 
LaTeX Source Files
Click here to download LaTeX Source Files: FSI_FinalRevision_JCP.tex

LaTeX Source Files
Click here to download LaTeX Source Files: FSI_FinalRevision_JCP.tex Click here to download LaTeX Source Files: FSI_FinatRevision_JCP.ex

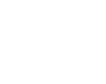

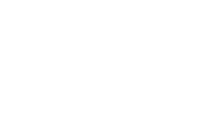
$\sqrt{2}$ $\sqrt{3}$ (1) (1) (1) (1)

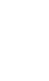
. . . . . . . . . . . . . 
LaTeX Source Files
Click here to download LaTeX Source Files: Figures_FinalRevision_JCP.tex

LaTeX Source Files
Click here to download LaTeX Source Files: Figures_FinalRevision_JCP.tex

Click here to download LaTeX Source Files: Figures_FinalRevision_JCP.tex

(n)
.

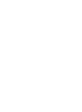

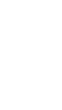
Click here to download LaTeX Source Files: Figures_FinalRevision_JCP.tex

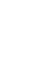
Click here to download LaTeX Source Files: Figures_FinalRevision_JCP.tex . (n) (n) (n) (n) . .

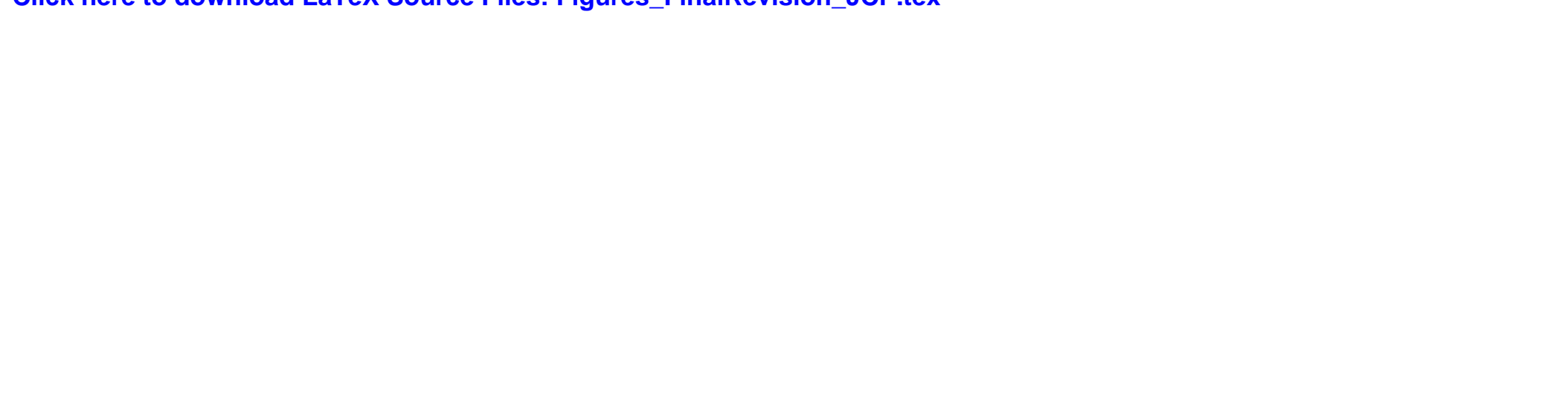

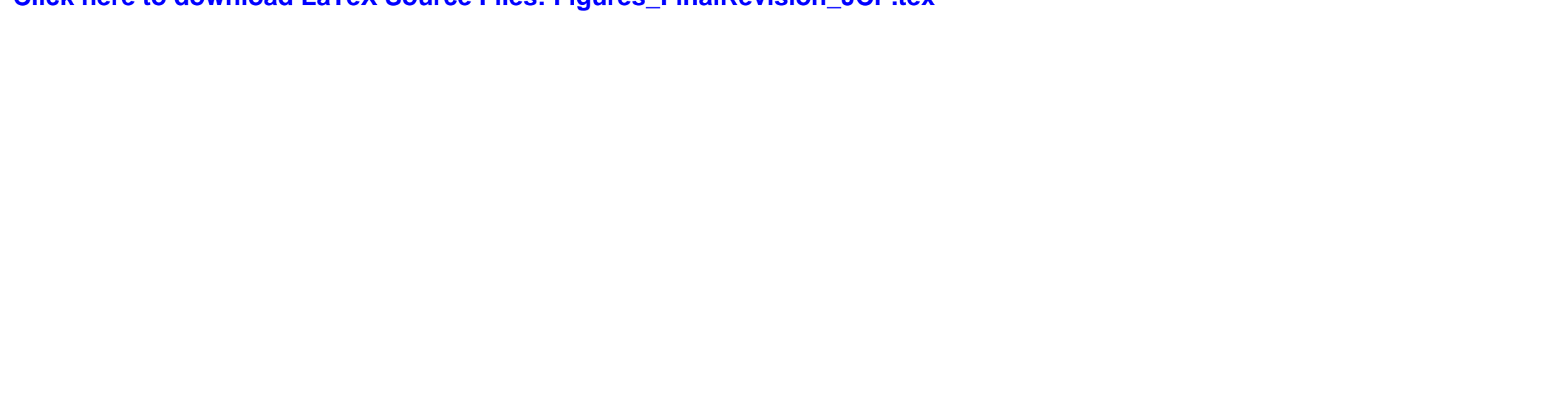

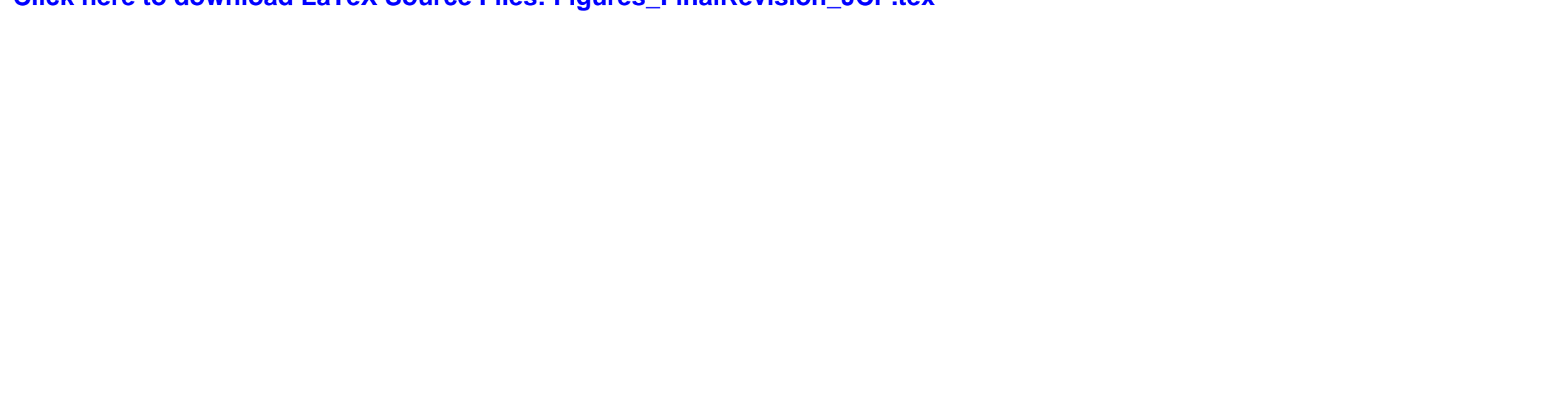

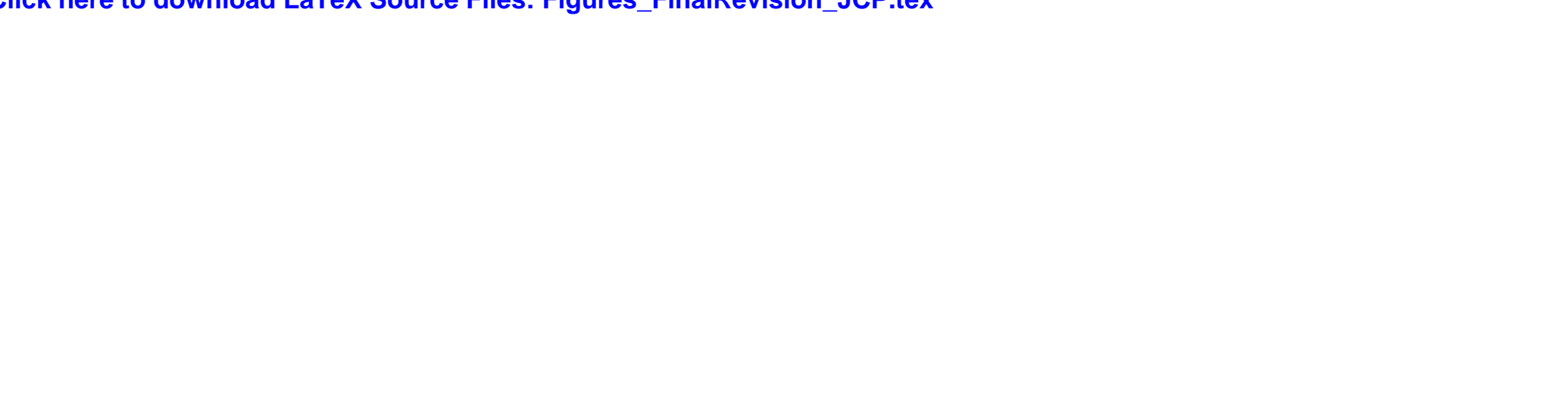

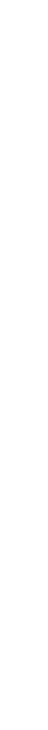

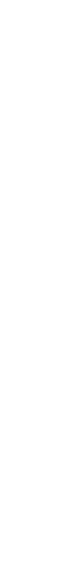

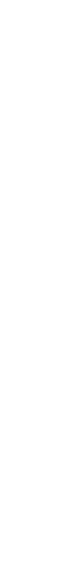

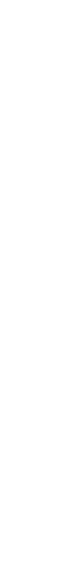

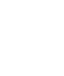

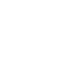
更 
LaTeX Source Files
Click here to download LaTeX Source Files: BiblioCite.bib

LaTeX Source Files
Click here to download LaTeX Source Files: BiblioCite.bib Click here to download LaTeX Source Files: Bibliocite bib

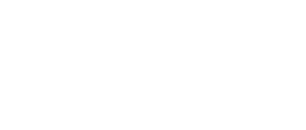

(1) .

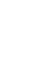

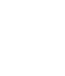
. . (1) (1) (1) (1) (1)

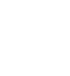
(1) (1) . . . . . . . . .

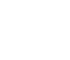

